\title{
Emotion Regulation and Cardiovascular Response to Emotion Provocation: Reappraisal versus Suppression
}

\author{
Casey E. Cavanagh \\ West Virginia University
}

Follow this and additional works at: https://researchrepository.wvu.edu/etd

\section{Recommended Citation}

Cavanagh, Casey E., "Emotion Regulation and Cardiovascular Response to Emotion Provocation:

Reappraisal versus Suppression" (2013). Graduate Theses, Dissertations, and Problem Reports. 426.

https://researchrepository.wvu.edu/etd/426

This Thesis is protected by copyright and/or related rights. It has been brought to you by the The Research Repository @ WVU with permission from the rights-holder(s). You are free to use this Thesis in any way that is permitted by the copyright and related rights legislation that applies to your use. For other uses you must obtain permission from the rights-holder(s) directly, unless additional rights are indicated by a Creative Commons license in the record and/ or on the work itself. This Thesis has been accepted for inclusion in WVU Graduate Theses, Dissertations, and Problem Reports collection by an authorized administrator of The Research Repository @ WVU. For more information, please contact researchrepository@mail.wvu.edu. 
Emotion Regulation and Cardiovascular Response to Emotion Provocation:

Reappraisal versus Suppression

Casey E. Cavanagh

Thesis submitted to the Eberly College of Arts and Sciences at West Virginia University in partial fulfillment of the requirements for the degree of

Master of Science

in

Psychology

Kevin Larkin, Ph.D. Chair

Barry Edelstein, Ph.D.

Julie Patrick, Ph.D.

Department of Psychology

Morgantown, West Virginia

2013

Keywords: emotion regulation, reappraisal, suppression, fear, psychophysiological responses 


\section{Abstract \\ Emotion Regulation and Cardiovascular Response to Emotion Provocation: \\ Reappraisal versus Suppression \\ Casey E. Cavanagh}

Emotion regulation is a process through which individuals alter the subjective, behavioral and physiological responses to emotional experiences. Within the emotion regulation model, reappraisal and suppression are two strategies that have been studied extensively. However, this research has largely ignored the effects of individual differences in emotion regulation when examining reappraisal and suppression. The purpose of this study was to examine individual differences in reappraisal and suppression (e.g., one’s preferred emotion regulation strategy) as well as examining the effects of instructional use of reappraisal and suppression. In the present study, participants, classified as habitual reappraisers and habitual suppressors, engaged in both reappraisal and suppression while viewing fear film clips. Measures of cardiovascular and affective responses to the film clips were obtained. A main effect of instructions was found for systolic and diastolic blood pressure (SBP and DBP respectively) with the reappraisal instructions resulting in lower blood pressure (BP) values than the suppression instructions. No additional main effects for the other dependent measures were observed. In addition, no interaction effects of habitual-strategy use (habitual reappraisers and suppressors) by instructional set (reappraisal vs. suppression) were observed.

The findings of a main effect of instructions for SBP and DBP are consistent with the literature in demonstrating that engagement in reappraisal produces lesser sympathetic activation as compared to engagement in suppression. The results demonstrate that reappraisal is associated with more positive cardiovascular benefits than suppression. These results extend previous work 
in demonstrating that the positive benefits of reappraisal are consistent when explored with an emotion that has received lesser attention in this literature (e.g., fear). Therefore, as this study and other recent studies have demonstrated, the effects of reappraisal appear to be consistent across negative emotions. This is a critical finding that increases the generalizability of reappraisal effects. Although habitual emotion regulation yielded no effects in the present study (as main effects or interacting with instructional set), low power limited detection of such effects. Future research is needed to examine the effect of habitual emotion regulation strategy on physiological responding to acute stress as well as responses to more natural stressors using ambulatory measurement strategies. 


\section{Table of Contents}

I. Introduction (pp. 1-26)
a. Types of Emotion (pp. 2-4)
b. Emotion, Stress, and Health (pp. 4-6)
c. Emotion Regulation (pp. 6-19)

i. Effect of Reappraisal on Response to Emotion Provocation (pp. 12-15)

ii. Effect of Suppression on Response to Emotion Provocation (pp. 15-19)

d. Purpose of the Current Study and Specific Aims (pp. 19-26)

II. Method (pp. 27-39)
a. Participants and Sample Size (pp. 27-30)
b. Measures (pp. 30-34)
c. Behavioral Observation (pp. 34-35)
d. Apparatus (p. 35)
e. Experimental Stimuli (pp. 35-37)
f. Procedure (pp. 37-39)

III. Results (pp. 40-47)

a. Data Preparation and Reduction (p. 40)

i. Blood Pressure (p. 40)

ii. Heart Rate (p. 40)

b. Preliminary Data Analyses (pp. 40-46)

i. Assessment of Stability of Cardiovascular Measures Obtained at Rest (pp. 40-41)

ii. Assessment of Comparable Reactivity across Film Segments (pp. 41-42) 
iii. Organization of Data and Testing Statistical Assumptions (pp. 42-43)

iv. Correlations between Dependent Variables with Demographic Variables (p. 43)

v. Behavioral Coding (pp. 43-44)

vi. Reactivity to Fear-eliciting Films (pp. 44-45)

vii. Manipulation Check for Emotion Regulation Instructions (pp. 45-46)

c. Primary Study Analyses (pp. 46-47)

IV. Discussion (pp. 47-60)

a. Strengths and Limitations (pp. 52-57)

b. Future Directions and Conclusions (pp. 58-60)

V. References (pp. 61-72)

VI. Table 1: Main Demographic Characteristics of SONA (Screening) Sample and Lab Sample (p. 73)

VII. Table 2: Additional Demographic Characteristics of SONA (Screening) Sample (p. 74)

VIII. Table 3: Preliminary Analyses of Resting Measures of SBP and DBP (p. 75)

IX. Table 4: Differences in Cardiovascular and Affective Reactivity between Neutral Film Clips (p. 76)

X. Table 5: Differences in Cardiovascular and Affective Reactivity between Fear Film Clips (p. 77)

XI. Table 6: Means and Standard Deviations for Covariates during Resting (Neutral Film) Periods (p. 78)

XII. Table 7: Means and Standard Deviations for Dependent Measures during Task (Fear Film) Periods (p. 79) 
XIII. Table 8: Univariate Correlations between Covariates and Demographic Variables (p. 80)

XIV. Table 9: Univariate Correlations between Dependent and Demographic Variables (p. 81)

XV. Table 10: Main Effects (Habitual Strategy and Instructional Set) and Interaction Effects for Habitual Reappraisers and Suppressors on Behavioral Observations (p. 82)

XVI. Table 11: Differences in Physiological and Affective Reactivity from Pre-Reappraisal Rest Periods to Reappraisal Task Periods (p. 83)

XVII. Table 12: Differences in Physiological and Affective Reactivity from Pre-Suppression Rest Periods to Suppression Task Periods (p. 84)

XVIII. Table 13: Main Effects and interaction Effects for Ease of Following Instructions and Ease of Regulating Emotions (p. 85)

XIX. Table 14: Main Effects (Habitual Strategy and Instructional Set) and Interaction Effects for Habitual Reappraisers and Suppressors Exposed to Fear Films while Reappraising and Suppressing: Heart rate and Blood Pressure (p. 86)

XX. Table 15: Main Effects (Habitual Strategy and Instructional Set) and Interaction Effects for Habitual Reappraisers and Suppressors Exposed to Fear Film while Reappraising and Suppressing: Heart Rate Variability Measures (p. 87)

XXI. Table 16: Main Effects (Habitual Strategy and Instructional Set) and Interaction Effects for Habitual Reappraisers and Suppressors Exposed to Fear Film while Reappraising and Suppressing: Measures of Affect (p. 88)

XXII. Figure 1: Mediation of the Stress - Disease Relation by the Acute Stress Response (p. 89) XXIII. Figure 2: Habitual Reappraisers (Upper Tertile and Lower Tertile Criterion) (p. 90) XXIV. $\quad$ Figure 3: Habitual Suppressors (Upper Tertile and Lower Tertile Criterion) (p. 91)

XXV. $\quad$ Figure 4: Habitual Reappraisers (Upper Tertile and Lower $50^{\text {th }}$ Percentile Criterion) (p. 92) 
XXVI. Figure 5: Habitual Suppressors (Upper Tertile and Lower 50 ${ }^{\text {th }}$ Percentile Criterion) (p. 93)

XXVII. Figure 6: Results of Block Randomization of Males and Females Categorized as Habitual Reappraisers and Habitual Suppressors into Conditions in Lab Phase (p. 94)

XXVIII. Figure 7: Procedure for Lab Phase (p. 95)

XXIX. Appendices (pp. 96-119)

a. Appendix A: Demographics Questionnaire (pp. 96-104)

b. Appendix B: Emotion Regulation Questionnaire (pp. 105-106)

c. Appendix C: Healthy Lifestyle Questionnaire (pp. 107-108)

d. Appendix D: Fear Survey Schedule-II (p. 109)

e. Appendix E: PANAS (pp. 110-111)

f. Appendix F: Post-film Questionnaire (p. 112)

g. Appendix G: Post-experiment Questionnaire (p. 113)

h. Appendix H: Editing Instructions (pp. 114-118)

i. Appendix I: Introduction to Film Clips (p. 119) 
The stress response, characterized by an individual's behavioral, cognitive, emotional, and physiological response to a real or perceived threat, can serve adaptive or maladaptive purposes (Ulrich-Lai \& Herman, 2009). Behaviorally, aggressive (fight), escape (flight), and inactivity (freeze) responses are commonly observed in response to stress. Cognitively, exposure to stress can lead to impaired concentration (Mohan, Sharma, \& Bijlani, 2011) and/or heightened vigilance to the source of the stress (Vedhara, Hyde, Gilchrist, Tytherleight, \& Plummer, 2000). Emotionally, a range of intense emotional experiences (e.g., anxiety, fear, anger, frustration, disgust, sadness) have been associated with the stress response. Finally, during the typical stress response, a range of physiological changes occur in the autonomic nervous system including alterations in the sympathetic and parasympathetic nervous systems. These physiological changes in the autonomic nervous system represent the most immediate response to stress with observable increases in cardiovascular arousal, as measured by heart rate and blood pressure (Ulrich-Lai \& Herman, 2009). As depicted in Figure 1 (Larkin, 2005), chronic activation of the stress response leads to mobilization of resources throughout the body, which can be detrimental to one's health when the stress response is prolonged, occurs frequently, or occurs intensely. Although initially adaptive, prolonged and substantial increases in cardiovascular arousal lead to increased risk of cardiovascular and cerebrovascular diseases (Ulrich-Lai \& Herman, 2009). In addition to the detrimental effects on the cardiovascular system, brain functioning is also affected during chronic activation of the stress response. For instance, the process of neurogenesis is inhibited during exposure to chronic stress (Sapolsky, Romero, \& Munck, 2000). Essentially, every organ system in the body (e.g., endocrine, respiratory, gastro-intestinal, immunological) is affected by the stress response, and therefore, represents a range of sites for physiological dysfunctions that occur as a consequence of exposure to stress. Understanding these detrimental 
effects of stress on the body has prompted several researchers to examine various moderators of the physiological stress response (Sapolsky, 2007; Ulrich-Lai \& Herman, 2009). After all, individuals vary in the magnitude and patterning of their stress responses, and consequently, exhibit differential predispositions for developing stress-related medical problems. As seen in Figure 1 (Larkin, 2005), these individual difference characteristics interact with exposure to stress to determine the magnitude and patterning of the acute stress response. The proposed study will examine one important individual difference characteristic, emotion regulation skill, and its influence on the acute stress response to emotion-provoking stimuli.

\section{Types of Emotion}

Before discussing the contemporary conceptualization of emotion regulation, a brief review of the emotion literature is necessary. Several early theories, including the James-Lange theory of emotions and the Cannon-Bard theory, offer contrasting conceptualizations of emotions. According to the James-Lange theory of emotions, physiological responses precede the experience of emotion in that emotions are simply the feelings that result from a pattern of physiological responses (James, 1884). In contrast, the Cannon-Bard theory of emotion does not specify a specific order for the experience of emotions. Rather, the Cannon-Bard theory suggests that physiological responses and the experience of emotions can occur simultaneously (Cannon, 1927). Schachter and Singer (1962) elaborated on these earlier theories by suggesting that the experience of emotion followed the cognitive appraisal of one's physiological reaction in a given environmental context. Physiological arousal in the context of a dangerous animal would result in the experience of fear, but the same physiological reaction in the context of a loved one would result in the experience of love. Consequently, contemporary models of emotions emphasize how emotions involve cognitive processes, which facilitate appraisal and one's response to a situation 
(Cole, Martin, \& Dennis, 2004). Further, these models typically discuss emotions in dichotomous terms, positive or negative emotions. Unlike positive emotions, negative emotions are linked to specific action tendencies such as, the fight-or-flight response (Fredrickson, Mancuso, Branigan, \& Tugade, 2000). Similarly, Gross and Thompson’s (2007) conceptualization of emotion regulation highlights the role of appraisal and behavioral response to an emotion-provoking situation.

Emotions, according to Gross and Thompson (2007), represent one state of affect. Using their conceptualization, affect is a superordinate category that consists of states of stress, emotion, mood, and motivational impulses (Gross \& Thompson, 2007). Accordingly, the 'modal model' of emotion consists of three core features. The first feature is the person-situation transaction, which compels an individual's attention to the source of stress. The second feature involves the appraisal of the transaction (i.e., as threatening, benign, or neutral). Finally, the third feature involves the systemic response evoked by emotions, which include an individual's subjective experience, behavioral expression, and physiological responses (Gross \& Thompson, 2007; Sheppes, Scheibe, Suri, \& Gross, 2011). This model of emotions recognizes that the experience of emotion can be either an adaptive or maladaptive component of the stress response.

Perspectives on emotions have radically evolved over the years with current research examining the adaptive and maladaptive nature of emotions (Gross, 1998b). Emotions play critical roles in facilitating decision-making, preparing motor responses, and are necessary for maintaining successful social interactions (Gross, 1998a). Their adaptive nature is evident when examining how contexts or situations can elicit emotions that serve to assist with coping (Gross \& Thompson, 2007). For example, a fear response elicited by confronting a shark while 
swimming typically results in an adaptive flight response and concomitant physiological activation. In contrast, emotional reactions can also be maladaptive, when they occur too frequently, too intensely, persist for long periods of time, or occur in the wrong context. Similar to the view of emotions as adaptive or maladaptive, emotions are also generally categorized as positive or negative. This categorization is based upon the responses evoked. For instance an emotion of anger, categorized as a negative emotion, is likely to result in physiological arousal as well as a subjective increase in negative valence. Therefore for the purposes of this study the term negative emotions will refer to maladaptive emotional reactions and positive emotions will refer to adaptive emotional reactions. Given the daily experience and expression of emotion as part of life, the balance of adaptive (positive) and maladaptive (negative) emotional reactions quite likely impacts one’s psychological and physical health.

\section{Emotion, Stress, and Health}

Research (Fredrickson \& Levenson, 1998) has indicated that positive emotions can serve adaptive functions in moderating relations between stress and health. For example, research has examined the health effects of positive emotions (Fredrickson \& Levenson, 1998). In a series of studies, Fredrickson and Levenson investigated whether positive emotions and spontaneous smiling produced lesser cardiovascular reactivity and more rapid recovery upon exposure to emotion-eliciting stimuli. The first study, which used undergraduates, found that exposure to positive films after an initial exposure to negative films was associated with more rapid cardiovascular recovery as compared to exposure to neutral or negative films after an initial exposure to a negative film. In a follow-up study, Fredrickson and Levenson continued their investigation of the effects of positive emotions on cardiovascular recovery using a sample ranging in age from 20 to 35. This study found that spontaneously smiling resulted in more rapid 
cardiovascular recovery from exposure to negative films as compared to nonsmilers. It was noted, however, that exposure to the negative film stimuli produced a range of emotions including, amusement, content, anger, disgust, fear, sadness and surprise. Based upon these findings, Fredrickson and Levenson (1998) argued that positive emotions and positive affect appear to have an 'undoing effect' on negative emotions, and therefore, positive emotions may play an important role in health by modulating stress responses and potentially reducing probability of disease onset.

Although research examining the effects of positive emotions on health is increasing, research has primarily examined the effects of negative affect and negative emotions on health. Several critical reviews (Gallo, Ghaed, \& Bracken, 2004; Kubzansky, \& Kawachi, 2000; Sirois \& Burg, 2003) have been conducted to examine this relation and concluded that negative affect and cognitions, such as that found in depression and anxiety, as well as negative emotions, such as hostility and anger, contribute to the etiology of coronary heart disease. Direct links for this association have been reported in studies, which have found that mental arousal and the experience of anger triggered ischemia in coronary artery disease patients (e.g., Gabbay et al., 1996). Furthermore, exposure to anger produced greater increases in heart rate in this study. Research of this type has clearly documented a relation between the experience and expression of anger and cardiovascular disease.

Research has similarly demonstrated the effects of negative emotions on health by examining a broader range of negative emotions, including sadness and frustration. One study (Gullette et al., 1997) found that negative emotions (e.g., frustration, sadness, tension) increased ischemia in patients with coronary artery disease, showing that negative emotions other than anger are associated with transient myocardial ischemia. Similar findings have also been 
observed in nonclinical samples. In one study, higher blood pressure was positively related to exaggerated responses to negative emotional stimuli (Nyklicek, Vingerhoets, \& Van Heck, 1996). In another study, individuals with high blood pressure were more likely to exhibit impaired ability to recognize both positive and negative emotions (McCubbin et al., 2011). Interestingly, research has also found that high blood pressure and the decreased subjective experience of emotions were associated with increased autonomic and circulatory system activation in response to stress (McCubbin, Surwit, \& Williams, 1985). In brief, hypertensive persons who denied the experience of emotions showed the largest stress responses. Research in this area indicates not only relations among negative emotion, stress, and health, but also demonstrates that the ability to regulate one's emotions may be a potential moderator of how exposure to emotion-provoking stimuli influences one’s cardiovascular response to stress.

\section{Emotion Regulation}

Emotion regulation is the process through which individuals respond to the experience of emotion-eliciting stimuli and resulting emotional responses (Gross, 1998a). Although related to the construct of coping, Gross (1998a, 1998b) contends that emotion regulation is a separate construct. Coping, which represents a broader category than emotion regulation, is a process through which an individual can respond to external problems or respond to internal emotions (Wang \& Saudino, 2011). Further, coping is generally considered context dependent, referring to the fact that it does not occur in the absence of stress, and time dependent in that coping does not occur prior to encountering a stressful event (Wang \& Saudino, 2011). Unlike coping, emotion regulation is primarily used to regulate internal emotions, which involves the expressive and physiological regulation of emotions occurring within the body at a given point in time (Gross, 1999). Additionally, emotion regulation, in contrast to coping, is thought to occur in response to 
both positive and negative emotions and can be employed prior to the experience of emotions (Wang \& Saudino, 2011). Like coping, specific emotion regulation strategies have been shown to influence both mental and physical health outcomes (Aldao, Nolen-Hoeksema, \& Schweizer, 2010; Denson, Grisham, \& Moulds, 2011; Gross, 1998a; Quartana \& Burns, 2010).

Various models of emotion regulation have been proposed, each acknowledging that emotion regulation is a process by which negative and positive emotions are increased, decreased, or maintained (Gross \& Thompson, 2007). For example, early psychoanalytic perspectives focused on regulation of anxiety via psychological defenses (Rapaport, 1953). Briefly, according to this perspective, anxiety was a primary emotional state associated with some discomfort, and defense mechanisms reflected rudimentary forms of emotion regulation that were aimed to reduce this discomfort (Gross, 1999). Stemming from the psychoanalytic tradition and the prevailing interest in the study of personality and individual differences, repression and rumination were highlighted as two important areas of study that became early precursors to today's literature on emotion regulation. Early definitions of repression emphasized the role of unconscious forces as mechanisms through which an individual prevented an event, or affect associated with an event, from reaching consciousness (Davis \& Schwartz, 1987). Modern definitions view repression as a mechanism involving inattention to threatening overt or covert stimuli that distract an individual from experiencing negative or unpleasant emotions or events (Gross, 1999). Although individuals high on measures of repressive coping have been shown to experience reduced intensity and expression of emotions when compared to persons low in repressive coping, they were more physiologically aroused (Davis \& Schwartz, 1987).

Rumination was another type of emotion regulation process that utilized attention (Gross, 1999). In contrast to repression, which involved limited attention to the source of threat, 
rumination involved dwelling on the threatening stimuli. Though the hope was that rumination assisted in problem solving and served to regulate emotions like sadness or other depressive feelings through engagement in conscious and effortful behavior, rumination usually increased the intensity of one's depressive feelings (Gross, 1999). In support of this perspective, research has demonstrated a strong relation between individuals who score high on measures of dispositional rumination and depressive symptoms (Just \& Alloy, 1997). Worry, a construct similar to rumination, functioned in much the same way when used for emotionally regulating anxiety (Dickson, Ciesla, \& Reilly, 2011). Findings from studies examining individual differences in rumination and repression provided the foundation for more recent empirical investigations of emotional regulation.

Contemporary models of psychopathology have highlighted the importance of emotion and emotional regulation in comprehending a range of human conditions, including anxiety, mood, and personality disorders (Gross, 1998a). In contrast to other models of dysfunctional human behavior that have focused on more enduring phenomena like mood, personality, or cognitive schemata, most of the empirical work examining emotion regulation has focused on the immediate responses to emotion-laden stimuli often presented in laboratory settings. According to this perspective, emotion regulation strategies are simply defined as response-tendencies (Gross, 1998a).

Response-tendencies are brief responses affecting the immediate experience and expression of emotion and associated autonomic and neuroendocrine system arousal (Lang, 1995). James (1884) contended that encountering life threatening situations elicits an adaptive emotional response. This adaptive response consists of both behavioral and physiological components. Using a dimensional perspective, Gross and other researchers conceptualize 
emotion regulation ranging from effortful to effortless, controlled to automatic, and conscious to unconscious processing (Cisler, Olatunji, Feldner, \& Forsyth, 2010; Gross, 1998a, 1999; McRae \& Gross, 2009). Over time, response-tendencies are acquired that reflect one’s typical ways of responding to emotion provocation. Gross's conceptualization represents only one model of emotion regulation, but has been studied extensively. Unlike other models of emotion regulation, Gross has identified specific emotion regulation strategies. As the focus of this thesis project is to examine the influence of individual differences in emotion regulation upon cardiovascular responses to emotion-laden stimuli, emotion regulation is conceptualized according to the Gross model. Two broad categories of emotion regulation strategies exist, with antecedent-focused strategies used prior to the onset of emotion and response-focused strategies used following the experience of emotion (Gross, 1998a). Within the antecedent-focused category of emotion regulation, there are four specific emotion regulation strategies: situation selection, situation modification, attentional deployment, and cognitive change. The response-focused category consists of only one emotion regulation strategy: response modulation.

Both antecedent and response-focused emotion regulation strategies are capable of increasing, decreasing, shortening, or lengthening the experience of emotions, and importantly, both produce effects on the subjective experience of, behavioral expression of, and physiological response to emotions (Gross, 1998b). An individual can alter the emotion generating process by utilizing situation selection to choose which specific situations to confront. For example, if an individual was afraid of being mugged while walking on the street at night, he or she could choose to never leave home after dark. By restricting nighttime travel, this individual eliminates contact with the feared stimuli and prevents the entire experience of anxiety that accompanies walking in town after dark. Utilizing the situation modification strategy involves altering specific 
aspects of a situation in an effort to regulate the accompanying emotion. Using this approach, rather than refusing to go out after dark, the fearful individual mentioned above may choose to leave home after dark, but only when accompanied by another person, or only while walking on well-lit streets, or only after earning a black belt in one of the martial arts. Each of these approaches represents a modification of the situation to alter its fear-evoking properties. Unlike situation selection and situation modification, attentional deployment consists of three separate strategies: concentration, distraction, and rumination (Gross, 1998a). Concentration and distraction involve increasing or decreasing attention to specific aspects of the situation, respectively, while rumination involves repeatedly thinking about a situation. Using distraction, the fearful individual described above may hum a song to him or herself while walking after dark or concentrate on counting the number of cars that drive down the street to regulate the intensity of their fear. Conversely, ruminating about all the horrible things that could happen to people after dark would be associated with an intensification of the emotional experience. The final antecedent-focused strategy, cognitive change, consists of cognitive reappraisal, which aims to modify the emotional impact of a given stimulus by altering the meaning of a stimulus or a situation (Gross \& John, 2003). Using cognitive reappraisal, the fearful individual might respond to his or her anxiety while walking after dark, by challenging maladaptive thoughts of imminent harm with thoughts like “nobody else is being mugged, so it really isn’t that dangerous” or “although being mugged can happen, it is a very unlikely event.”

In contrast to the antecedent-focused strategies, response modulation is the singular response-focused strategy in emotion regulation. Response modulation consists of suppressing the expression of emotion, and inhibits the typical behavioral response to emotional stimuli (Gross \& John, 2003). Using the individual fearful of walking alone at night, a response 
modulation strategy would be to force him- or herself to walk at a comfortable pace without excessive vigilance to one's surroundings despite feeling afraid. Importantly, as each of these strategies demonstrates, emotion regulation can occur at various points in the emotion generation process, with each strategy utilizing different cognitive and/or behavioral skills.

Despite the identification of five emotion regulation strategies, most of the research on emotional regulation examines the strategies of reappraisal and suppression only. This focus on reappraisal and suppression is evidenced by the fact that the Emotion Regulation Questionnaire (ERQ), the predominant method used for purposes of measuring the effect of emotional regulation strategies on mental and physical health, is comprised of two subscales labeled Reappraisal and Suppression (Gross \& John, 2003). Since these two emotional regulation strategies are used frequently in everyday life and can be manipulated in the lab, they are typically measured in studies of this type (Gross \& John, 2003). Furthermore, reappraisal and suppression represent types of antecedent and response-focused strategies, respectively (Gross \& John, 2003). Gross (1999) reviewed past research, mainly stemming from the psychoanalytic literature, that indicated suppression of emotions produced anxiety and negative effects on mental and physical health. Consequently, researchers currently view reappraisal of emotions as a more adaptive response to emotional provocation than suppression (Gross, 1999). Given the historical importance of these two methods of regulating emotion and their roles in contemporary models of emotion (Gross, 1998b; Gross, 1999; Gross \& Levenson, 1993), it is important to examine the experiential, behavioral, and physiological responses to emotion provocation associated with both reappraisal and suppression. In the next section, I will provide an overview of the experimental literature contrasting the emotional regulation strategies of reappraisal and suppression. 


\section{Effects of Reappraisal on Response to Emotion Provocation}

Research examining reappraisal has focused primarily on the effects of reappraisal on psychological rather than physical health (Gross, 1998b). In brief, cognitive change (cognitive reappraisal) strategies have been associated with reduced stress and appear to be an important adaptive emotion regulation strategy when confronting stressful situations (Moore, Zoellner, \& Mollenholt, 2008). In addition, research has demonstrated that the use of cognitive change strategies also resulted in positive effects on psychological health (Gross, 1998b). When measuring the effects of reappraisal on the subjective experience and behavioral expression of emotions, research by Gross (1998b; 1999) has demonstrated that reappraisal has different effects on the experiences of both negative and positive emotions than suppression. In studies of this type, researchers have typically used a disgust-eliciting film as an emotional stimulus, such as a film involving a medical procedure like amputation of a limb. However, using a disgust stimulus limits the generalizability of these findings to other emotions (Gross, 1998b; Gross \& Levenson, 1993). Recent research has expanded to use a range of emotional stimuli, including both positive (e.g., amusement or surprise) and other negative emotions (e.g., anger, fear, or sadness) (Gross \& Levenson, 1995; Kreibig, Wilhelm, Roth, \& Gross, 2007). Despite the limitations of using only a disgust stimulus, research examining reappraisal has consistently shown reappraisal to be associated with reductions in emotionally-expressive behavior and the intensity of the subjective experience of emotion (Gross, 1998b, 1999).

Although the effects of reappraisal on the experience and expression of emotion have been consistently demonstrated using numerous methods of assessment, it is important to examine the effects of reappraisal on psychophysiological responses to emotion provocation. In contrast to self-report methods of assessing emotion, assessment of physiological responses are 
less susceptible to potential response biases associated with subjective ratings of emotional intensity and duration. Research examining the effects of reappraisal upon psychophysiological responses associated with emotion has focused mainly on examining alterations of sympathetic and parasympathetic activation, using measures of finger pulse amplitude, finger temperature, skin conductance, heart rate, and respiration. Early research found that the psychophysiological responses to emotion provocation of undergraduates with a mean age of 21 years in a reappraisal condition were similar to those in a control condition (Gross, 1998b), a finding replicated in subsequent empirical work (Butler, Egloff, Wilhelm, Smith, Erickson, \& Gross, 2003). Recent research, however, contradicts these earlier findings, suggesting that reappraisal affects psychophysiological responses to emotion provocation by producing reductions in autonomic arousal.

Reappraisal has been shown to elicit less physiological arousal when compared to control or "watch" conditions (Wolgast, Lundh, \& Viborg, 2011). This study included participants with a mean age of 27.4 years and found that the watch condition yielded greater skin conductance levels and greater corrugator electromyographic (EMG) responses than those in the reappraisal condition. Another study, using college students with a mean age of 21.8 years, examined reappraisal of stressors, in response to a speech preparation task, and found that reappraisal resulted in lower total peripheral resistance (TPR) reactivity but greater cardiac output (CO) reactivity than control and ignore conditions (Jamieson, Nock, \& Mendes, 2011). TPR is a measure of vasoconstriction/vasodilation, and CO is the amount of blood ejected from the heart each minute. Current research has provided additional evidence of adaptive cardiovascular functioning associated with reappraisal. Denson et al. (2011) examined heart rate variability (HRV) among undergraduate women with a mean age of 20.2 years and found that HRV 
increased among individuals in a reappraisal condition (indicating increased parasympathetic activity) as compared to those in suppression and control conditions. Additionally, the researchers found that ratings of anger were highest among individuals in the suppression condition. This finding is particularly important since low HRV is associated with poor cardiovascular health, suggesting that reappraisal is a successful emotion regulation strategy to use in situations evoking anger. Combining the findings of lower TPR and greater CO from Jamieson et al.'s (2011) study and higher HRV from Denson et al.’s (2011) study, it would seem that individuals engaged in reappraisal exhibited more adaptive cardiovascular functioning in response to stress than those engaged in suppression. Despite the early negative results from studies examining the influence of reappraisal on psychophysiological responses to stress, the most recent research (Denson et al., 2011; Jamieson et al., 2011; Stemmler, 1997) examining reappraisal has produced significant findings demonstrating reductions in psychophysiological arousal, in particular when using reappraisal during social situations.

Examining the use of reappraisal in social situations is an area of particular interest as this suggests that the effect of a particular emotion regulation strategy may vary depending on the context within which the emotion is induced. For example, Butler et al. (2003) compared the effects of reappraisal and suppression using a social interaction in college-aged women. In the study, pairs of women were exposed to upsetting films with one partner in each dyad engaging in reappraisal, suppression, or acting naturally (control condition). Butler et al. predicted that partners of those in the reappraisal condition would demonstrate less physiological activation than those of partners in the suppression condition or those of partners instructed to act naturally. Results demonstrated that partners of those in the suppression condition exhibited greater increases in blood pressure than those in the reappraisal condition, providing evidence that 
reappraisal was a more effective emotion regulation strategy when encountering complex social situations. The effectiveness of reappraisal in social situations was also supported by additional research examining compassion-focused and benefit-focused reappraisal of a past interpersonal offense among students with a mean age of 18.9 years (vanOyen Witvliet, Knoll, Hinman, \& DeYoung, 2010). Both reappraisal strategies resulted in more positive and less negative valence affective states than a control condition, with specific increases shown in ratings of happiness and joy and decreases shown in ratings of anger. Compassion-focused reappraisal demonstrated reduced affective arousal as measured by heart rate in comparison to benefit-focused appraisal. These studies provide additional support for the finding that reappraisal is associated with dampened physiological emotion responses when compared with either control or suppression instruction conditions. Given the breadth of emotions evoked in these studies and the range of stimuli used to elicit emotion, it is unclear to what extent the effects of emotion regulation strategies may be emotion-specific. Additional research is clearly needed to determine whether the influence of reappraisal on psychophysiological response to stress varies depending upon the emotion examined in the study and the methods used to induce the emotion. Despite these limitations in the aforementioned literature, the majority of empirical work in this area has demonstrated that the emotional regulation strategy of reappraisal is associated with reductions in the subjective, behavioral, and psychophysiological experience of emotions.

\section{Effects of Suppression on Response to Emotion Provocation}

Although empirical work examining the psychophysiological responses associated with reappraisal has increased in recent years, research examining the effects of suppression has exploded in recent years. Results from studies examining suppression suggest that suppression may be more effective at reducing the behavioral responses to emotion provocation than 
reappraisal. For example, multiple studies have found suppression to produce less expressive behavior when compared with control and reappraisal conditions (Gross, 1998b, Gross \& Levenson, 1993; Roberts, Levenson, \& Gross, 2008). In two studies using male and female undergraduates ranging in age from 17 to 23 , individuals assigned to suppression conditions demonstrated lesser body movement than those in control conditions when exposed to emotional provocation (burn and amputation films); however, participants in the suppression conditions still reported high disgust ratings (Gross \& Levenson, 1993). Another study, which only included female undergraduates, demonstrated similar reductions in expressive behavior among individuals in a suppression condition compared to individuals in a control condition (Gross \& Levenson, 1997). Interestingly, decreased expressive behavior was observed when exposed to both positive and negative emotions, whereas reappraisal of positive emotions produced greater behavioral expressions of emotion (Gross \& John, 2003).

Research examining the effects of suppression on the subjective experience of emotions has been mixed. Although researchers have hypothesized that suppression should demonstrate similar reductions in the subjective experience of emotions, this hypothesis has only been partially supported. In one study, suppression produced no changes in the subjective experience of emotions (Gross \& Levenson, 1993); however, additional studies found that suppression produced a decreased subjective experience of some emotions, including pain, pride, amusement, and fear (Dunn, Billotti, \& Dalgleish, 2009; Gross \& Levenson, 1997). Similar reductions in the subjective experience of emotion were not observed for other emotions such as disgust and sadness (Gross \& Levenson, 1997). These contradictory findings are particularly interesting, as much of the research examining the effects of suppression has relied on exposing study participants to emotions of disgust, fear, or anger. 
The positive benefits of suppression (i.e., reduced behavioral expression and subjective experience) are sharply contrasted with the commonly observed increased psychophysiological arousal. This indicates that suppression may be a less adaptive emotion regulation strategy than reappraisal, at least regarding physiological reactivity to stress. In contrast to the effects of reappraisal, researchers have hypothesized that suppression should result in increased sympathetic nervous system responses and decreased somatic nervous system activity (DanGlauser \& Gross, 2011; Gross, 1998b; Gross \& Levenson, 1993). Indeed, research has demonstrated greater sympathetic nervous system activity for individuals in suppression conditions when compared to reappraisal and control conditions. In particular, several studies found that suppression participants demonstrated greater reductions in finger pulse amplitude and finger temperature and increased levels of skin conductance in comparison with participants in reappraisal conditions (Gross, 1998b; Gross \& Levenson, 1993). The decreased finger pulse amplitude and finger temperature as well as the increased levels of skin conductance indicate that suppression was associated with greater vasoconstriction, demonstrating increased sympathetic nervous system activation. However, decreased somatic nervous system activity and decreased heart rate responding were not observed in these studies. This may have resulted from a floor effect, because other research has demonstrated decreased somatic nervous system activity and heart rate responding associated with suppression (Dan-Glauser \& Gross, 2011; Gross, 1998b; Gross \& Levenson, 1993, 1997). For example, Gross and Levenson (1993) found greater reductions in somatic nervous system activity and heart rate responding for individuals in a suppression condition as opposed to those in a control condition. According to Gross and Levenson, the greater heart rate deceleration observed among individuals in the suppression condition suggested cardiac-somatic coupling (i.e., the tendency for heart rate changes to mirror 
changes in muscle tension) or emotion specific autonomic activity. Despite the observed reductions in somatic activity associated with suppression, research has typically found suppression to be associated with increased sympathetic nervous system activation that has been demonstrated across both exposure to positive (amusement) and negative (sadness) emotions (Gross \& Levenson, 1997), indicating that suppression is likely to result in autonomic arousal independent of the specific emotion being regulated.

More direct evidence of the negative effects of suppression on cardiovascular health is apparent when considering results from research using cardiovascular measures, such as blood pressure. Quartana and Burns (2010) found that college students ( $M_{\text {age }}=19.6$ years) in a suppression condition demonstrated higher systolic blood pressure responses to two stressors when compared to those in a control condition. In addition, the study demonstrated that systolic blood pressure responses were higher for the second stressor presented in the study for only those in the suppression condition, suggesting that suppression has a cumulative and delayed effect. Importantly, research suggests that the effects of suppression on psychophysiological responses are similar across ethnic groups of college students ( $M_{\text {age }}=20.8$ years) (Roberts et al., 2008). Roberts et al. found that individuals in a suppression condition exhibited increased sympathetic activation, as indicated by increased systolic and diastolic blood pressure, in response to disgust stimuli (burn and amputation films). The same pattern of results was observed among African American participants. These results demonstrate that ethnicity did not moderate the direction of psychophysiological responses observed when engaged in suppression as a strategy of emotion regulation.

The damaging cardiovascular effects observed when engaging in suppression were further evident in other research (Butler, Wilhelm, \& Gross, 2006). In this study, Butler et al. 
examined HRV in female college students $\left(M_{\mathrm{age}}=20.0\right.$ years $)$ comparing reappraisal, suppression, and uninstructed (control) conditions. Participants in the reappraisal condition experienced greater positive emotions as compared to the uninstructed and suppression conditions, which experienced greater negative emotions. Although Butler et al. observed increased HRV during suppression conditions, the increases in HRV were even greater in reappraisal conditions, providing further evidence that reappraisal is associated with better physiological indicators of cardiovascular health than other forms of emotion regulation. The evidence regarding the effects of suppression on health is less conclusive than the evidence of the positive effects of reappraisal, but demonstrates that suppression does exhibit some positive benefits, at least regarding behavioral responses to emotion provocation. In sum, research suggests that the emotional regulation strategy of suppression elicits unique patterns of physiological response to emotion provocation, and that such response profiles are less likely to reduce physiological responding than reappraisal.

\section{Purpose of the Current Study and Specific Aims}

Emotion regulation research has expanded rapidly in recent years as researchers attempt to elucidate the processes underlying emotion regulation. In addition, research has also focused on examining the psychophysiological responses to emotion provocation associated with distinct emotion regulation strategies and the relations between these emotion regulation strategies and health. From the literature examined in the introduction above, it would seem that regulating emotion via reappraisal versus suppression is associated with distinctive physiological response patterns. Although mixed findings exist, most studies show that reappraisal is associated with a dampened physiological response to emotion provocation and suppression is associated with 
lesser behavioral responding to emotion provocation that is associated with increased physiological responding.

The primary purpose of the proposed study was to examine two strategies of emotion regulation, reappraisal and suppression, as a potential moderating influence of the stress-disease relation, with specific attention paid to the magnitude of the acute physiological response to stress. There are two general approaches that can be considered for this purpose. On the one hand, emotion regulation strategy can be conceptualized as an individual difference variable, in which persons acquire preferred, typical, or habitual strategies for regulating emotion.

Alternatively, emotion regulation can be modified through the use of instructional sets in which study participants are guided to use one strategy over the other, regardless of their typical or habitual response tendencies. To examine the effects of habitual versus instructional use of reappraisal and suppression, all participants in the current project engaged in both reappraisal and suppression. Participants were categorized as habitual reappraisers/low suppressors or as habitual suppressors/low reappraisers. Using a repeated measures design ensured comparison of habitual and instructional use. Each group (habitual reappraisers and suppressors) engaged in each emotion regulation strategy (reappraisal and suppression) one time, allowing for a comparison of individual's responses to both emotion regulation strategies. There is evidence that individuals acquire typical or habitual emotion regulation strategies. In general, the habitual use of reappraisal for regulating emotion has been shown to be associated with reductions in the experience and expression of negative emotions and increased experience and expression of positive emotions (Gross \& John, 2003). This has important implications for social functioning, as individuals who habitually use reappraisal are more likely to share positive and negative emotions with those in their social support system than individuals who habitually use 
suppression (Gross \& John, 2003). The positive benefits of habitual use of reappraisal have also been shown to be evident in high reappraisers, who have closer relationships with their peers than those who use reappraisal infrequently (Gross \& John, 2003). In addition, research has found that habitual use of reappraisal is associated with greater well-being, as high reappraisers report fewer depressive symptoms, greater self-esteem, higher rates of life satisfaction, and were more optimistic than high suppressors (Gross \& John, 2003; McRae, Jacobs, Ray, John, \& Gross, 2012). In contrast, high frequency of suppression for regulating emotion is associated with decreases in the experience and expression of positive emotions (Gross \& John, 2003). High frequency of suppression is also associated with increases in the experience of negative emotions, possibly resulting from feelings of inauthenticity as suppression masks the genuine expression of emotions (Gross \& John, 2003). As peers were able to detect inauthenticity portrayed by suppressors, individuals who engaged in habitual use of suppression were more likely to have decreased social support than those who use reappraisal habitually (Gross \& John, 2003). High frequency of suppression has also been associated with impaired well-being, as evidenced by greater depressive symptoms, lower self-esteem, lower life satisfaction ratings, and less optimism (Gross \& John, 2003).

The Emotion Regulation Questionnaire (Gross \& John, 2003) is a popular instrument that measures habitual use of reappraisal and suppression and was used in this study to identify participants who were habitual reappraisers and those who were habitual suppressors. Research has demonstrated that habitual use of reappraisal is related to mood repair, indicating that habitual reappraisers appear to be more successful at mood repair than habitual suppressors (John \& Gross, 2004). Habitual use of reappraisal is also related to positive reinterpretation and growth (John \& Gross, 2007). Conversely, habitual suppression, as a result of suppressing 
emotions, is associated with experiencing a feeling of incongruence between their inner self and outer behavior (John \& Gross, 2004). Therefore, habitual use of suppression appears to be related to inauthenticity and avoidance (John \& Gross, 2004; 2007).

Despite the frequent use of the Emotion Regulation Questionnaire in the literature, most studies examining the psychophysiological influences of reappraisal and suppression have done so by altering task instructions without regard to an individual's habitual emotion regulation strategy. As such, some participants in studies of this type are assigned to engage in a familiar emotion regulation strategy and others are assigned to use a strategy with which they are less familiar. The failure of authors of these studies to assess and account for this potentially important individual difference factor may lead to the emergence of inconsistent results across studies.

The purpose of this study was to examine psychophysiological responses associated with reappraisal and suppression in regulating the emotional experience of fear. Rather than adopting a range of emotions, the study examined only one. Previous findings (Kreibig et al., 2007) indicate that psychophysiological responses to emotions are variable, even when examining differences in responses among negative emotions. In one study, Kreibig et al. (2007) found differences in activation of the autonomic nervous system in response to fear and sadness stimuli with respect to both the intensity and response specificity of psychophysiological responses. Specifically, fear produced an accelerated heart rate, whereas sadness produced a decelerated heart rate. Additionally, sadness produced no change in diastolic blood pressure from resting baseline as compared to fear that produced increases in diastolic blood pressure. As the results from Kreibig et al.’s (2007) suggest, psychophysiological responses to emotions demonstrate distinctive response specificity. Examining response specificity is critical in determining whether 
the effects of emotion regulation strategies are consistent across emotions. Only a few studies (Gross \& Levenson, 1995; Kreibig et al., 2007; Wolgast et al., 2007) have examined psychophysiological responses of emotion regulation strategies using a fear stimulus. Therefore, fear stimuli were used in the current study to contribute to this under-investigated emotion in the emotional regulation literature.

In contrast to previous studies, this study contributes to the emotion regulation literature by examining the psychophysiological responses to fear of individuals identified as habitual reappraisers and those identified as habitual suppressors. Using a within subjects factor, both habitual reappraisers and habitual suppressors were instructed to engage in both strategies in a single laboratory session. As such, the psychophysiological responses of habitual reappraisers and habitual suppressors were compared during engagement in familiar emotion regulation strategies (i.e., habitual reappraisers instructed to reappraise and habitual suppressors instructed to suppress) and less familiar emotion regulation strategies (i.e., habitual reappraisers instructed to suppress and habitual suppressors instructed to reappraise). The unique aspect of this study design is that it will assist in determining if the physiological effects associated with each specific emotion regulation strategy were more strongly influenced by the habitual emotion regulation characteristics of the individual or the instructions provided to engage in specific emotion regulation strategies. Importantly, examining habitual reappraisers and habitual suppressors enables us to focus on a presumably important individual difference characteristic, which may be necessary to understand the relations among stress, emotion regulation, and health. Studies examining habitual use of emotion regulation strategies are limited, and as such, methods for categorizing participants as reappraisers or suppressors are far from standardized. One study (Mauss, Cook, Cheng, \& Gross, 2007) compared high and low reappraisers and 
another study (Menchola, 2008) examined habitual versus instructional use of reappraisal and suppression. Mauss et al. (2007) used scores on the Reappraisal Subscale of the ERQ to categorize participants as high reappraisers by selecting those from the upper tertile and as low reappraisers by selecting those from the lower tertile. Participants included female students with 33 participants categorized as high reappraisers $\left(M_{\text {reappraisal score }}=36.6\right)$ and 33 as low reappraisers $\left(M_{\text {reappraisal score }}=22.8\right)$. Menchola (2008) recruited undergraduate students and grouped participants into three groups: reappraisers, suppressors, and a non-preference group. Each group then engaged in reappraisal, suppression, and a watch (control) conditions. Participants were defined as reappraisers if they scored in the $75^{\text {th }}$ percentile on the Reappraisal Subscale of the ERQ and the $25^{\text {th }}$ percentile on the Suppression Subscale of the ERQ. Conversely, to be categorized as suppressors, participants scored in the $75^{\text {th }}$ percentile on the Suppression Subscale of the ERQ and the $25^{\text {th }}$ percentile on the Reappraisal Subscale of the ERQ. Participants in the non-preference group scored between the $25^{\text {th }}$ and $75^{\text {th }}$ percentile on both subscales of the ERQ. Mean scores (and standard deviations) of the three groups on the Reappraisal and Suppression scales of the ERQ were as follows:

\begin{tabular}{lll} 
Group & Reappraisal Score & Suppression Score \\
\hline Reappraisers & 36.28 & 7.63 \\
Non-Preference & 27.57 & 14.65 \\
Suppressors & 19.10 & 21.15 \\
\hline
\end{tabular}

Results from Mauss et al.’s (2007) and Menchola’s (2008) studies have important implications for examining habitual use of emotion regulation strategies. Mauss et al. (2007) found that high reappraisers demonstrated more adaptive cardiovascular responses than high 
suppressors, including greater cardiac output and ventricular contractility and lower total peripheral resistance. Menchola compared habitual and instructional use of reappraisal and suppression using corrugator and orbicularis EMG and skin conductance and found that instructions to use reappraisal or suppression produced significant differences in physiological responses, but no differences were observed between study participants who were habitual reappraisers or suppressors. Despite the use of only a few physiological measures, Menchola found that instructions to reappraise resulted in decreased corrugator muscle activity, whereas suppression instructions produced no changes in corrugator activity. No differences were observed on measures of skin conductance responses. Menchola hypothesized that this lack of observed effects resulted from the decision to use images from the International Affective Picture System (IAPS) as emotion provoking stimuli as opposed to the use of more active emotion-evoking stimuli like films. It is quite likely that the static IAPS images (negative, neutral, and positive) represented very minimal emotion provocations and that the use of fearevoking films would produce more observable differences in physiological activity. Through use of a similar research design as the study by Menchola, the current study extends her work by using film exposures to fearful stimuli and a broad array of cardiovascular measures to evaluate changes in physiological responding to emotion.

Prior research has not examined the cardiovascular responses of habitual reappraisers and habitual suppressors. As suppression is associated with poor cardiovascular health (e.g., Quartana \& Burns, 2010), it is important to examine cardiovascular parameters most closely linked with cardiovascular health outcomes. Given that both high blood pressure and reduced HRV are associated with an increased risk for cardiovascular impairment, it is important to examine these specific response parameters to emotion provocation. Furthermore, as reappraisal 
is associated with more positive cardiovascular responses than suppression (e.g., (Jamieson et al., 2011; Wolgast et al., 2011), it is important to know if habitual use of reappraisal serves as a buffer against the negative effects of instructional suppression measured in laboratory settings. Therefore, this study contributes to the growing literature examining emotion regulation and health.

Since research has not previously examined individual differences in cardiovascular reactivity to stress associated with these two types of emotion regulation, it was difficult to predict how habitual reappraisers would respond when asked to reappraise or suppress, and how habitual suppressors would respond when asked to reappraise or suppress. Congruent with the findings of Menchola (2008) on muscle activity, it was possible that engagement in reappraisal and suppression via laboratory instructions would result in characteristic physiological response differences, regardless of habitual use of specific emotion regulation strategies (i.e., increased cardiovascular arousal would be observed during suppression instructions regardless of habitual emotion regulation strategy). This outcome was called the Instructional Set main effect. Alternatively, it was possible that habitual reappraisers would exhibit lesser cardiovascular arousal than habitual suppressors to both film presentations, regardless of instructional set (i.e., Habitual Strategy main effect). Finally, it was possible that habitual use of emotional regulation strategies would moderate the nature of these autonomic response differences so that individuals' pattern of responding would differ when engaging in their typical mode of emotion regulation versus engaging in an atypical mode of emotion regulation (i.e., interaction effect). Because of the limited data available from the literature, no specific hypotheses were made. 


\section{Method}

\section{Participants and Sample Size}

The sample size for the proposed study was determined using G*Power 3.1.3 (Faul, Erdfelder, Buchner, \& Lang, 2009), by entering the study design as an a priori, ANCOVA: Fixed effect, main effects, and interactions with an effect size $f$ of $0.4, \alpha=.05$, and power $=80 \%$. This effect size was determined from previous studies comparing heart rate responses of individuals exposed to different emotion regulation strategies in which several large and medium effect sizes were reported (Denson et al., 2011; Hofmann, Heering, Sawyer, \& Asnaani, 2009; Mauss, Cook, Cheng, \& Gross, 2007; Quartana \& Burns, 2010). In one study (Mauss et al., 2007), the eta squared for the main effect of heart rate was .58, and in another study (Denson et al., 2011), Cohen’s $d$ for heart rate variability for participants in a reappraisal condition was .37. Based upon these parameters, a sample size of 52 participants was judged to be capable of detecting large effect sizes for purposes of examining hypotheses in the proposed study. However, in order to obtain an equal number of habitual reappraisers and habitual suppressors in each order of task presentation, we aimed to recruit 56 participants from the screening sample.

A total sample of 995 participants $\left(M_{\text {age }}=21.6\right.$ years, $\left.S D=63.5\right)$, consisting of 764 females and 228 males, completed the survey through the SONA system (see Tables 1-2 for demographics of SONA and lab samples). Three participants did not disclose their sex. All participants received extra credit for completing the screening phase of the study. Of the entire screening sample, 329 participants (33\%) were deemed eligible for the lab phase of the study. Participants were identified as habitual reappraisers if they scored in the upper tertile on the reappraisal subscale $(M=35.64, S D=3.69)$ and the lower tertile on the suppression subscale $(M$ $=9.50, S D=2.56)$. Similarly, participants were identified as habitual suppressors if they scored 
in the upper tertile on the suppression subscale $(M=19.69 S D=2.75)$ and the lower tertile on the reappraisal subscale $(M=21.25, S D=5.74)$. Using this criterion 141 habitual reappraisers and 146 habitual suppressors were identified during the initial six months of data collection (see Figures 2 and 3). Initially114 habitual reappraisers and 111 habitual suppressors were declared eligible and invited for participation in the lab phase of the study. Participants in the lab phase of the study were entered into a lottery with the chance of winning \$25 (i.e., 10 participants from the sample would win $\$ 25$ each). During this period of data collection, 17 habitual reappraisers and 20 habitual suppressors were scheduled for the lab phase of the study. One habitual reappraiser and four habitual suppressors did not show up for the laboratory session and failed to reschedule, and one habitual reappraiser discontinued the study after viewing the second neutral film clip. Although the participant, who discontinued the study, did not withdraw authorization permitting analysis of their data, this data was not included in the main analysis.

After several months of data collection, the eligibility criterion and incentives were adjusted because of low participation rates. Instead of scoring in the lower tertile on the reappraisal or suppression subscale, participants were then declared eligible if they scored in the lower $50^{\text {th }}$ percentile. Habitual reappraisers were then defined as participants scoring in the upper tertile on the reappraisal subscale $(M=35.53, S D=3.58)$ and the lower $50^{\text {th }}$ percentile on the suppression subscale $(M=9.73, S D=2.63)$. A similar criterion was used to identify habitual suppressors: those scoring in the upper tertile on the suppression subscale $(M=19.44$., $S D=$ 2.72) and the lower $50^{\text {th }}$ percentile on the reappraisal subscale $(M=22.06, S D=5.89)$. Using this criterion an additional 69 habitual reappraisers and 78 habitual suppressors were identified (see Figures 4 and 5). An additional 51 additional habitual reappraisers and 53 additional habitual suppressors were invited to participate in the lab phase of the study. In addition, the incentive for 
participation was increased so that 10 participants would each win $\$ 50$. Following the change in eligibility criterion and incentives, an additional four participants were scheduled (two habitual reappraisers and two habitual suppressors) for the lab phase of the study. One from each group completed the session, with the remaining person failing to show up or reschedule.

A total of 141 habitual reappraisers and suppressors were declared ineligible to participate in the lab phase of the study. Specifically, 49 participants were ineligible based upon major chronic health conditions (e.g., hypertension; diabetes), 33 were ineligible based upon use of nicotine, 22 were ineligible based upon significant missing data, 20 were ineligible based upon use of medications affecting heart rate or blood pressure, and 17 were ineligible based on presence of a serious mental health problem.

Each eligible participant received two invitations to participate in the lab phase of the study. Participants were contacted through email if they included their email address on the survey or through the SONA messaging system. Of the 329 eligible participants, 41 (12.5\%) accepted invitations to participate in the lab phase. Habitual reappraisers were block randomized into one of two conditions: (a) reappraisal instructions during the first fear film followed by suppression instructions during the second fear film, or (b) suppression instructions during the first fear film followed by reappraisal instructions during the second fear film. Habitual suppressors were also block randomized into one of the same two conditions. Results of block randomization are shown in Figure 6. The final sample consisted of 16 habitual reappraisers (12 females and four males) and 17 habitual suppressors (13 females and four males). One participant (habitual reappraiser) discontinued the study following the second neutral film clip. Although this participant discontinued the study following the second neutral film clip, the 
participant's physiological and affective responses from the first and second neutral film clip and the first fear film clip were included in data analysis, when possible.

\section{Measures}

Demographic Form. A short demographic form used in previous studies in the Behavioral Physiology laboratory (e.g., Melanko, 2011, Prentice, 2009) was used in this study. This questionnaire included items pertaining to age, gender, height, weight, race/ethnicity, year in school, and parental socioeconomic status. The form also included general questions about participants’ health behaviors (See Appendix A).

Emotion Regulation Questionnaire (ERQ). The ERQ is a self-report assessment of typical emotion regulation strategies, consisting of 10 items using a 7-point Likert-type scale, ranging from 1 (Strongly disagree) to 7 (Strongly agree) (Gross \& John, 2003). The ERQ is comprised of two subscales assessing an individual's use of cognitive reappraisal (i.e., Reappraisal Scale; 6 items) and expressive suppression (i.e., Suppression Scale; 4 items) strategies. Alpha reliabilities across four experiments averaged .79 for the Reappraisal Scale and .73 for the Suppression Scale (Gross \& John, 2003). Stability coefficients assessing test-retest reliability across three months were .69 for both scales. The ERQ has also demonstrated convergent and discriminant validity. Two scales from the COPE, Reinterpretation and Venting, demonstrated good convergent validity as reappraisal was related to reinterpretation $(\beta=.43, p<$ $.05)$ and suppression was inversely related to venting $(\beta=-.13, p<.05)$. As for discriminant validity, neither reappraisal $(\beta=.11, p>.05)$ nor suppression $(\beta=-.09, p>.05)$ were shown to be associated with Crowne and Marlow’s social desirability scale. Additional evidence of discriminant validity was evident when examining the standardized beta coefficients on the Big Five Inventory for reappraisal (ranging from $\beta=-.20$ to $\beta=.13, p<.05$ ) and the standardized 
beta coefficients on the Big Five Inventory for suppression (ranging from $\beta=-.41$ to $\beta=.11, p<$ .05). Although reappraisal and suppression converged slightly with the subscales on the Big Five Inventory, the standardized beta coefficients demonstrate that reappraisal and suppression represent different constructs than the broad personality dimensions measured by the Big Five Inventory. Further, additional evidence of discriminant validity is demonstrated when examining the lack of correlation between measures of emotional regulation and cognitive ability (Wonderlic Intelligence Test, Form IV part A and B and the SAT) (Reappraisal, $\beta=.02, p>.05$; Suppression, $\beta=.09, p>.05$ ) (The ERQ is depicted in Appendix B).

Healthy Lifestyle Questionnaire. The Healthy Lifestyle Questionnaire is a self-report assessment consisting of 30-items measuring a number of health behaviors. Although it was originally designed to utilize a dichotomous, yes/no response format, the questionnaire was adapted in a previous study to utilize a Likert-type format $(1=$ Rarely/Never to $4=$ Always/Almost Always) (Melanko, 2011). The Healthy Lifestyle Questionnaire consists of 11 subscales: Physical Activity, Nutrition, Managing Stress, Avoiding Destructive Habits, Practice Safe Sex, Adopting Safety Habits, Knowing First Aid, Personal Health Habits, Using Medical Advice, Being an Informed Consumer, and Protecting the Environment. Higher scores on the Healthy Lifestyle Questionnaire are associated with more healthy behaviors and lower scores are associated with less healthy behaviors. Although limited psychometric evaluation has been conducted on this questionnaire, items are face valid. Also, items on this questionnaire resemble items on other health behavior measures, such as the Behavioral Risk Factor Surveillance System (BRFSS, 2009). In a previous study, alpha reliabilities for the total score was .76 (Melanko, 2011), indicating moderate internal consistency to the items comprising this questionnaire (See Appendix C). 
Fear Survey Schedule-II (FSS-II). The Fear Survey Schedule-II is a 51 item self-report questionnaire assessing several domains of fear: specific phobias, social phobias, agoraphobic situations, and fearful situations unrelated to phobias (Antony, 2001). Respondents are asked to rate the extent to which a stimulus or experience causes fear or unpleasant feelings on a 7-point Likert-type scale $(1=$ None, 7 = Terror $)$ with higher scores indicating greater fear. Using KuderRichardson formula 20, the overall internal consistency coefficients are reported very high at .94. In addition, the scale exhibits convergent validity through its correlation with other measures of emotionality and anxiety and discriminative validity through its poor correlation with measures of introversion and extroversion (Antony, 2001). Specifically, the FSS-II demonstrated moderate convergent validity with Taylor's Manifest Anxiety Scale (TMAS), a measure of trait anxiety, with a correlation coefficient of .55 for females. Discriminant validity was established by examining correlations between the FSS-II and Ford's scale, a measure of social desirability. Geer demonstrated a weak negative relation between Ford's scale and the FSS-II with correlation coefficients ranging from -.23 for males and -.27 for females. Two additional items were added to this measure for this study including, "watching scary movies” and "watching horror films” to increase the content validity of this measure for purposes of this study (See Appendix D).

Positive and Negative Affect Schedule (PANAS). The PANAS is a 20 item self-report questionnaire measuring both positive and negative affect (Watson, Clark, \& Tellegen, 1988). Individuals are instructed to respond using a 5-point Likert-type scale (1 = very slightly or not at all to 5 = extremely). Positive affect (PA) reflects a pleasurable engagement, with high PA characterized by a state of enthusiasm and alertness, whereas negative affect (NA) reflects unpleasurable engagement and is characterized by a state of subjective distress (Crawford \& Henry, 2004; Watson et al., 1988). The PANAS has been used in numerous research studies 
since its development (Crawford \& Henry, 2004). Importantly, the PANAS can utilize different temporal instructions ranging from moment instructions (“at the present moment”) to the past year (“during the past year”) and in general (“on average”). As this study required the PANAS to be administered four times, we used the moment instructions. Watson et al. (1998) demonstrated moderate to strong internal reliability for both the PA and NA scales. The alpha reliabilities of the PANAS for PA have been shown to range from .86 for the year instructions to .90 for the today instructions. The alpha reliability for PA for the moment instructions was reported as .89. For NA, the alpha reliabilities have ranged from .84 for the year instructions to .87 for the today, past few weeks, and general instructions. The alpha for NA with moment instructions was reported as .86. The PANAS has also demonstrated adequate test-retest reliability over a one week interval, with test-retest coefficients of .81 for NA and .79 for PA. Watson et al. (1998) also examined convergent validity of the PANAS. The PANAS demonstrated good convergent validity with several measures of affect, including the Hopkins Symptom Checklist, the Beck Depression Inventory, and the State-Trait Anxiety Inventory Sate Anxiety Scale (A State). For the NA scale of the PANAS, correlation coefficients with these three affect measures ranged from .51 to .74. These measures also demonstrated lesser associations with the PA scale of the PANAS with correlation coefficients ranging from -.19 to .36, providing further evidence of divergent validity (Watson et al., 1988). The PANAS is shown in Appendix E.

Post-film Questionnaire. This short form, which includes questions adapted from Kreibig et al.’s (2004) study, was used to assess participants’ feelings (pleasantness and intensity) following each film clip. This form also includes yes/no questions about participants’ behaviors during each film clip (e.g., “Did you look away during the film clip?”) (See Appendix F). 
Post-experiment Questionnaire. This short form includes questions about participants' experiences in the experiment (e.g., "Please briefly describe your experience in this study.”) (See Appendix G).

\section{Behavioral Observation}

Two behavioral observation measures were obtained in this study to ensure that the length of exposure to the fear film clips was consistent across participants. In addition, the inclusion of this observational measure provided a manipulation check to ensure that participants did not use additional emotion regulation strategies, like attentional deployment to distract one’s attention from the emotional film segments. Therefore, all study sessions were recorded using the web camera on the Dell laptop computer, which was also used for viewing the film clips. The investigator viewed each video excerpt and completed two behavioral observations: the number

of times the participants looked away from the computer screen for less than five seconds (Look Away) and the number of times participants looked away from the computer screen for more than five seconds (Sustained Look Away). Twenty-two percent of video excerpts were doublecoded to assess inter-rater reliability. Inter-rater reliability was determined by calculating intraclass correlation coefficients between raters’ counts of Look Away and Sustained Look Away occurrences. The intraclass correlation coefficient for Look Away $\left(r_{\mathrm{IC}}=.91\right)$ indicated strong inter-rater agreement, whereas the intraclass correlation coefficient for Sustained Look Away $\left(r_{\mathrm{IC}}=.66\right)$ indicated moderate inter-rater agreement. The strong inter-rater agreement for Look Away demonstrates that this behavioral observation was a reliable measure of attentional deployment. The moderate inter-rater agreement for Sustained Look Away may have occurred because this behavioral observation required a more complex coding system, which included a temporal component. However, in conjunction with the more reliable Look Away coding 
strategy, the moderate inter-rater agreement was still considered appropriate to analyze, albeit with caution.

\section{Apparatus}

Two psychophysiological devices were used to measure cardiovascular responses to the emotion stimuli used in this study. A Polar Model RS800 Heart Rate Monitor (Lake Success, New York) was used to measure heart rate as well as obtain measures of heart rate variability. This device employs a sensor attached to the participants' chest and transmits ECG signals to a wristwatch type receiver. The receiver was connected to a computer located in a separate room that was monitored by the experimenter throughout the study. The Polar Monitor has been validated for measuring heart rate in psychophysiological studies (Goodie, Larkin, \& Schauss, 2000). Continuous heart rate data was recorded in computer files for later spectral analysis for obtaining two measures of heart rate variability (HRV): High frequency (HF) HRV (reflecting parasympathetic nervous system activity) and low frequency (LF) HRV (reflecting both sympathetic and parasympathetic nervous system activity.

Blood pressure [both systolic (SBP) and diastolic blood pressure (DBP)] was measured using a Suntech AccuTracker Ambulatory Blood Pressure Monitor (Morrisville, NC). This device employs an occluding cuff positioned over the brachial artery attached to an electronic processing unit that controls cuff inflation/deflation and stores blood pressure values for later downloading to a computer. The AccuTracker has been shown to measure blood pressure reliably (Nelson, Weber, Elliott, Zebrauskas, \& Murphy, 1988).

\section{Experimental Stimuli}

The four film clips selected for this study are: two clips from Alaska's Wild Denali (Rohlfing, 1997) (601 seconds, Clip 1, and 599 seconds, Clip 2), I Know What You Did Last 
Summer (Chaffin, Feig, Moritz, \& Gillespie, 1997) (639 seconds, Clip 3), and I Still Know What You Did Last Summer (Beasley, Chaffin, Feig, Moritz, \& Cannon, 1998) (674 seconds, Clip 4). Multiple studies have demonstrated that clips 1 and 2 evoke neutral feelings and that clips 3 and 4 evoke feelings of fear (Kolodyazhniy, Kreibig, Gross, Roth, \& Wilhelm, 2011; Kreibig, Ferstl, \& Roth, 2004; Kreibig et al., 2007). Presentation of these clips were divided into two sets, Set A (Clips 1 \& 3) and Set B (Clips 2 \& 4). All participants viewed each of the four film clips once. Editing instructions for the four films, used by Kreibig et al. (2004) are shown in Appendix H. In addition, as suggested by Kreibig et al. (2004), each film was preceded by a brief overview of the plot (Appendix I). The presentation of the films, Set A and Set B was block randomized within habitual reappraisers and habitual suppressors.

The length of these film segments is appropriate given that accurate measurements of frequency-based measures of HRV require at least a five minute duration. In validating these film clips, Kreibig et al. (2004) obtained ratings of respondents using the following three criteria: discreteness, discriminability, and similarity. Kreibig assessed discreteness by examining whether the target emotion (i.e., fear) received high ratings compared to other emotions. Discriminability examined if participants were capable of correctly classifying the target emotion that each film clip was meant to evoke (i.e., fear). Similarity was assessed through use of correlations comparing the two film clips per emotion (i.e., comparing fear film clip to fear film clip) to determine if each film clip evoked the target emotion (i.e., fear) at similar ratings.

Neutral stimuli. The two film clips from Alaska's Wild Denali (1 \& 2) have been shown to evoke comparable neutral emotional responses (Kolodyazhniy et al., 2011; Kreibig et al. 2004; Kreibig et al., 2007). Ratings of the discreteness of the first neutral film clip found higher ratings of amusement, happiness, enthusiasm, and interest than other emotions as compared to other film 
clips (clips targeting fear, sadness, neutral emotions). Importantly, both neutral film clips also evoked higher ratings for enthusiasm as compared to other negative affect emotions, including anger, disgust, fear, and sadness. The only difference in discreteness between the two neutral film clips was the finding of higher ratings for enthusiasm as compared to happiness and interest that occurred for film clip 2. Discriminability criteria were used to further demonstrate the validity of the neutral film clips. For the first and second neutral film clips, participants' emotion self-report ratings demonstrated high levels of correct classification, $71.4 \%$ and $100 \%$ respectively.

Fear stimuli. The two film clips, I Know What You Did Last Summer (Clip 3) and I Still Know What You Did Last Summer (Clip 4), have been demonstrated to evoke fear in previous studies (Kolodyazhniy et al., 2011; Kreibig et al., 2004; Kreibig, et al., 2007). Clip 4 evoked higher ratings of fear than all positive affect emotions, except interest. In addition, Clip 4 evoked higher ratings of fear as compared to other emotions, including anger, happiness, sadness, distress, guilt, shame, and nervousness. Clip 3 also evoked higher ratings of fear than happiness, sadness, guilt, jitteriness, and nervousness. The validation of the two fear clips was also demonstrated through discriminability as participants’ correctly classified the target emotion (fear) of the two films in $100 \%$ of the cases. The correlation between the fear ratings of the two films clips was $0.72(p<0.05)$ demonstrating similarity between these two clips.

\section{Procedure}

All participants reviewed an IRB approved cover letter prior to beginning the SONA survey. The screening sample completed a set of questionnaires through SONA, including: a Demographic Screening Questionnaire (see Appendix A), the Emotion Regulation Questionnaire 
(see Appendix B), the Healthy Lifestyle Questionnaire (see Appendix C), and the Fear Survey Schedule II (see Appendix D).

Participants who were eligible were contacted through email or the SONA message system to schedule an individual appointment for the laboratory part of this study. Upon arriving to the laboratory, participants completed IRB approved consent forms and had the opportunity to ask questions about the study. Participants were informed prior to the start of the study that they would be recorded throughout the experiment. The experimenter then obtained the participants' height and weight, which was used to calculate body mass index (BMI). Participants were instructed to attach the heart rate monitor and then the experimenter attached the blood pressure cuff to their upper arm. During a brief adaptation period, the experimenter verified the signal from the Polar HR Monitor and verified the accuracy of BP measures. After verifying the accuracy of physiological measures, participants were instructed to watch a neutral film clip, from either Set A or Set B. Instructions for watching the neutral film clip were adapted from previous studies (Aldao \& Mennin, 2012; Gross, 1998b); in brief, participants were instructed to “Watch this film clip carefully.” Order of instructions was counterbalanced however; order of clip presentation was partially counterbalanced.

Participants then watched the fear film from Set A or B associated with the neutral scene they had just viewed. Prior to viewing of this clip, participants were instructed to reappraise or suppress emotions during film presentation using instructions based on those used in a previous study (Gross (1998b). Brief reminders to reappraise or suppress were presented three times during the film clip. The reappraisal instructions were as follows:

We will now be showing you a short film clip. It is important to us that you watch the film clip carefully, but if you find the film too distressing, just say "stop." This time, 
please try to adopt a detached and unemotional attitude as you watch the film. In other words, as you watch the film clip, try to think about what you are seeing objectively, in terms of the technical aspects of the events you observe. Watch the film clip carefully, but please try to think about what you are seeing in such a way that you don't feel anything at all (p. 227)

The instructions for suppression were as follows:

We will now be showing you a short film clip. It is important to us that you watch the film clip carefully, but if you find the film too distressing, just say "stop." This time, if you have any feelings as you watch the film clip, please try your best not to let those feelings show. In other words, as you watch the film clip, try to behave in such a way that a person watching you would not know you were feeling anything. Watch the film clip carefully, but please try to behave so that someone watching you would not know that you are feeling anything at all (p. 227-228)

Participants then watched the remaining neutral and fear film clips (Set A or Set B), receiving the remaining set of instructions to either reappraise or suppress emotions during the fear film segment. Both neutral film clips served as resting periods for participants.

Participants viewed all film clips on a Dell laptop computer. Following presentation of each film clip, participants completed the PANAS (Appendix E) to rate affect and a Post-film Questionnaire (Appendix F). Participants also completed a Post-experiment Questionnaire (Appendix G) about their experience in the study. Measurement apparatuses were detached and participants were debriefed regarding the purpose of the study. See Figure 7 for an outline of the lab phase procedure. 


\section{Results}

\section{Data Preparation and Reduction}

Blood Pressure. Preliminary data preparation was conducted to remove invalid blood pressure values using the criteria proposed by Marler et al., (1988). Using this procedure any excessively high and/or low blood pressure values and those characterized by unacceptable pulse pressures were removed. Pulse pressure was calculated by subtracting diastolic blood pressure from systolic blood pressure. If pulse pressure values were less than $30 \mathrm{~mm} \mathrm{Hg}$, the associated systolic and diastolic blood pressures values were eliminated and not used in subsequent analyses. Twenty-one pulse pressure values were found to be less than $30 \mathrm{~mm} \mathrm{Hg}$ and therefore the associated systolic and diastolic blood pressure values were removed. Missing or removed blood pressures were imputed where possible by using the closest blood pressure value or an average of the two closest blood pressure values; 24 SBP and 24 DBP values were imputed representing only $2.02 \%$ of the SBP data and $2.02 \%$ of the DBP data collected in this study.

Heart Rate. Heart rate recordings from the Polar monitor were scanned using software that accompanies this device and questionable values were removed. The Kubios HRV Analysis Software program also detects invalid heart rate recordings and was used as a second pass to remove erroneous values prior to calculating HRV variables. The standard spectrum setting for analyzing normal HRV, an interpolation rate of $4 \mathrm{~Hz}$ (Tarvainen \& Niskanen, 2008), and a low level of artifact correction, was applied to the heart rate recordings. Niskanen, Tarvainen, Rantaaho, and Karjalainen (2002) have demonstrated that the Kubios HRV Analysis Software program provides valid measures of HR and HRV.

\section{Preliminary Data Analyses}

\section{Assessment of Stability of Cardiovascular Measures Obtained at Rest. Prior to} averaging cardiovascular measures within each rest and task period one-way repeated measures 
ANOVAs were conducted on SBP and DBP, values collected during the first rest period (see Table 3) to determine if any resting values were different from the others and potentially removed from additional analyses. The ANOVA on SBP was significant, $F(3,24)=3.74, p<$ .05 , and mean contrasts revealed a significant difference between the first SBP value $(M=121.3$ $\mathrm{mm} \mathrm{Hg}, S D=17.76)$ and second SBP value $(M=116.9 \mathrm{~mm} \mathrm{Hg}, S D=15.93), F(1,26)=7.18, p$ $=.01$. This effect was considered to be due to a novelty effect of having the first blood pressure taken and therefore, the first SBP value was eliminated and not included in the calculation of mean SBP for that period. There were no significant differences between DBP values during the first rest period. This strategy could not be used for HR or HRV because only one measure of each was obtained per rest and task period. Next, SBP and DBP values within each experimental period were averaged to obtain a single measure of SBP and a single measure of DBP for each period.

Assessment of Comparable Reactivity across Film Segments. To demonstrate that both neutral tasks and both fear tasks elicited comparable responses, paired-samples t-tests were conducted on measures of cardiovascular (SBP, DBP, HR, HF HRV, and LF HRV) and affective (PA and NA) responses (see Tables 4-5). For the neutral film clips, LF HRV was lower during Neutral Film Clip I $(M=1642.4 \mathrm{~Hz}, S D=1256.40)$ compared to Neutral Film Clip II $(M=$ 2936.4 Hz, $S D=2741.54), t(29)=-2.35, p=.03$. Because higher levels of HRV are associated with increased vagal (i.e., parasympathetic) activity (Berntson et al., 1997), Neutral Film II evoked significantly higher levels of autonomic arousal than Neutral Film I. In all other respects, Neutral Film clip I and II were comparable.

A significant difference was found when comparing SBP reactivity between Fear Film I and Fear Film II. SBP $(M=122.3$ mm Hg, $S D=13.84)$ was higher during Fear Film II as 
compared to Fear Film I $(M=119.02$ mm Hg, $S D=14.92), t(28)=-2.84, p=.008$ (see Table 5). The increased SBP during Fear Film II indicated that there was greater cardiovascular activation during Fear Film II and possibly could be interpreted to mean that Fear Film II evoked a higher level of the target emotion (fear). However, no significant differences were observed for any other dependent variable. The finding of these few significant differences in reactivity between the neutral and the fear film clips suggests that there were some small differences in the level of physiological arousal evoked by each film clip. Previous research has established that both neutral film clips evoked comparable neutral emotional responses and that both fear films produced comparable ratings of fear (Kolodyazhniy et al., 2011; Kreibig et al. 2004; Kreibig et al., 2007). Potential implications of these findings will be addressed in the discussion.

Organization of Data and Testing Statistical Assumptions. All dependent variables (HR, SBP, DBP, HF HRV, LF HRV, PA, and NA) and behavioral observations (Look Away, Sustained Look Away) were recoded and organized in preparation for the study's main analyses. This involved recoding them into four experimental periods: the rest period that preceded the instruction to reappraise (Pre-Reappraisal), the rest period that preceded the instruction to suppress (Pre-Suppression), the task period that included instructions to reappraise (Reappraisal), and the task period that included instructions to suppress (Suppression). Using these new variables, the assumptions for ANCOVA were examined. All dependent measures were examined for normality. Tabachnick and Fidell (2012) suggest that z-scores of 3.2 or greater are skewed. In the current data set, because of significant skewness, HRV data, PA and NA were log transformed. Blood pressure values were square root transformed because of moderate skewness. See Tables 6-7 for means and standard deviations of transformed and untransformed covariates and dependent variables. 
In examining the assumptions for ANCOVA, it was determined that two assumptions were violated for some variables: the assumption of normality and the assumption of homogeneity of variance. As discussed previously, problems with normality within the data were addressed through data transformations. Levene's test was then conducted on the transformed data to examine the assumption of homogeneity of variance for each dependent variable. Levene's test showed that only one variable violated the assumption of homogeneity of variance: NA during reappraisal $(p=.006)$. Although assumptions for ANCOVA were violated for only one study variable, the main analysis, a series of mixed factors ANCOVAs, was conducted. ANCOVAs have been reported to be relatively robust to violations of these assumptions (Field, 2013).

Correlations between Dependent Variables with Demographic Variables. Prior to conducting the main analysis, univariate correlations were calculated to examine the relations between four demographic variables (age, sex, or BMI, health behaviors) and each of the dependent variables (see Tables 8-9). Because sex was significantly correlated with systolic and diastolic blood pressure, sex was included as a covariate in the main analysis for blood pressure. BMI was significantly correlated with $\mathrm{HR}$, so BMI was included as a covariate in the main analysis for HR. No consistent relations were observed among remaining demographic variables and dependent variables.

Behavioral Coding. Prior to conducting the main study analyses, the two behavioral observation measures (Look Away, Sustained Look Away) were subjected to a 2 x 2 [Habitual Use (Reappraisers, Suppressors) X Situational Instructions (Reappraisal, Suppression)] mixed factors analysis of covariance (ANCOVA), covarying the associated behavioral parameter measured during neutral film clips. If a particular emotion regulation strategy or instructional set 
resulted in differential attention to the film clips, it would need to be covaried in the primary study analyses. The results of these ANCOVAs revealed no main effect of habitual use (habitual reappraisers and suppressors), no main effect of instructional set (reappraisal and suppression), and no interaction effect of habitual use by instructional set (see Table 10). Therefore, the behavioral observations were not included in the main analysis as additional covariates.

Reactivity to Fear-eliciting Films. It was also important to demonstrate that the film clips used in this study actually elicited physiological and affective reactions among study participants prior to examining the main study hypotheses. To do so, paired-samples t-tests were conducted to compare dependent measures during each film clip with the corresponding value obtained during the neutral film clip presentation that preceded it. As exposure to the film clips were expected to evoke the target emotion (i.e., fear), then there should be evidence of sympathetic activation when comparing physiological measures during rest periods to physiological measures during task periods. Similarly, evidence of affective reactivity should be demonstrated by either reductions in PA from rest periods to task periods or increases in NA from rest periods to task periods. Results of these analyses are presented in Tables 11-12 and show that significant SBP, DBP, and NA reactivity from the rest period to the task period was observed for reappraisal instructions. SBP was significantly higher during the reappraisal task period $(M=119.7 \mathrm{~mm} \mathrm{Hg}$, $S D=14.44)$ than SBP during the pre-reappraisal rest period $(M=118.5 \mathrm{~mm} \mathrm{Hg}, S D=15.31)$, $t(26)=-2.13, p=.04$. DBP was significantly higher during the reappraisal task period $(M=80.8$ $\mathrm{mm} \mathrm{Hg}, S D=8.80)$ than DBP during the pre-reappraisal rest period $(M=78.9 \mathrm{~mm} \mathrm{Hg}, S D=$ 10.30), $t(26)=-2.08, p=.05$. There was also evidence of affective reactivity, which was evident by the significant increase in NA during the reappraisal task period $(M=16.78, S D=6.43)$ 
compared to NA during the pre-reappraisal rest period $(M=10.42, S D=1.00), t(31)=-6.46, p \leq$ .001 .

The same pattern of reactivity was observed for the suppression instructions. SBP and DBP showed significant reactions from the pre-suppression rest period to the suppression task period. SBP was significantly higher during the suppression task period $(M=122.8 \mathrm{~mm} \mathrm{Hg}, S D$ $=17.22)$ than SBP during the pre-suppression rest period $(M=118.5 .5 \mathrm{~mm} \mathrm{Hg}, S D=15.31)$, $t(27)=-2.43, p=.02$. DBP was significantly higher during the suppression task period $(M=81.4$ $\mathrm{mm} \mathrm{Hg}, S D=8.83)$ than DBP during the pre-suppression rest period $(M=78.0 \mathrm{~mm} \mathrm{Hg.,} S D=$ 8.37), $t(27)=-4.52, p \leq .001$. Finally, the increase in NA from the pre-suppression rest period $(M$ $=10.6, S D=1.17)$ to NA during the suppression task period $(M=18.2, S D=7.36), t(32)=-$ 7.42, $p \leq .001$, was significantly different. All other paired-samples t-tests did not reveal significant differences (see Tables 11-12).

Cumulatively these results provided evidence that exposure to fear film clips produced physiological and affective reactivity as determined by some of the measures used in this study. These results also revealed that measures of HR, HRV, and PA were relatively unaffected by exposure to these fearful movie clips. These findings suggest that differences in HR, HRV, or PA response to the tasks due to emotion regulation strategies may prove difficult to demonstrate, given that no reactivity to the fear-eliciting tasks on these variables was observed at all.

Manipulation Check for Emotion Regulation Instructions. Finally, as a manipulation check, we examined participants' ease of following the emotion regulation instructions. Although using self-report data to determine adherence to reappraisal and suppression instructions may not be the optimal method, this method is typically employed in the literature (Denson et al., 2011; Gross, 1998b). For reappraisal, in particular, no other alternative method 
has been developed. In contrast, adherence to suppression instructions can be measured using behavioral observations, if instructions specify the purpose is to suppress the expression of emotions. However, because of the video quality in the present study, it was not possible to examine adherence to suppression instructions using a behavioral coding system. As a result of the complexity involved in measuring adherence to emotion regulation instructions, self-reported adherence was examined. Two mixed factors ANOVAs compared habitual reappraisers’ and suppressors' ease of following emotion regulation instructions (reappraisal and suppression) using data from the Post Film Questionnaire. No significant differences were observed. These data indicate that reappraisal and suppression instructions were comparable in terms of ease of implementing the emotion regulation strategies (see Table 13).

\section{Primary Study Analyses}

The primary study analysis involved conducting a series of 2 x 2 [Habitual Use (Reappraisers, Suppressors) X Situational Instructions (Reappraisal, Suppression)] mixed factors analysis of covariance (ANCOVA) on SBP, DBP, HR, HF HRV, LF HRV, PA, and NA. Covariates were the corresponding values measured during the associated neutral film periods. All analyses were conducted using a .05 level of confidence to determine statistical significance. Through each analysis, significant results would support the Habitual Strategy Main Effect, the Instructional Set Main Effect, or the Moderating Effects hypotheses proposed in the introduction to this study.

Results of these analyses, shown in Tables 14-16, showed a main effect for Instructional Set (reappraisal vs. suppression) for SBP, $F(1,22)=3.55, p=.04$, and DBP, $F(1,22)=3.37, p=$ .04. SBP was lower during the reappraisal task period $(M=119.7 \mathrm{~mm} \mathrm{Hg}, S D=14.44)$ than SBP during the suppression task period $(M=122.8 \mathrm{~mm} \mathrm{Hg}, S D=17.22)$. Similarly, DBP was lower 
during the reappraisal task period $(M=80.8 \mathrm{~mm} \mathrm{Hg}, S D=8.80)$ than DBP during the suppression task period $(M=81.4 \mathrm{~mm} \mathrm{Hg}, S D=8.83)$. The finding of lower SBP and DBP during engagement in reappraisal in contrast to suppression is consistent with the literature, demonstrating the positive benefits of reappraisal (Butler et al., 2003; Denson et al., 2011; Wolgast et al., 2011). For SBP no significant main effects for Habitual Use, $F(1,22)=.98, p=$ .33, or significant interaction effects, $F(1,22)=.32, p=.73$, were observed. In addition for DBP, no significant main effects for Habitual Use, $F(1,22)=.09, p=.77$, or significant interaction effects, $F(1,22)=.32, p=.73$, were observed.

No additional main effects for Instructional Set (reappraisal vs. suppression), Habitual Use (habitual reappraisers vs. habitual suppressors), or their interaction was found for the other dependent variables (HR, LF HRV, HF HRV, PA, and NA). Although a main effect of instructional set was found for SBP and DBP is consistent with the literature, no additional main effects were observed.

\section{Discussion}

The purpose of this study was to examine the psychophysiological effects of habitual and instructional use of emotion regulation strategies. Specifically, we compared cardiovascular responses (SBP, DBP, HR, LF HRV, and HF HRV) and affective responses (PA and NA) among habitual reappraisers and suppressors when engaged in reappraisal and suppression. The results of the present study are consistent with previous research in finding differences in physiological responses between reappraisal and suppression when participants are instructed to engage in these emotional regulation strategies (Butler et al., 2003; Denson et al., 2011; Quartana \& Burns, 2010). 
The main analysis revealed a significant Instructional Set main effect for SBP and DBP, with the lowest blood pressure reactions observed during reappraisal task periods. The finding of a significant effect for instructions is consistent with previous research demonstrating that reappraisal results in less sympathetic activation as compared to suppression (Butler et al., 2003; Denson et al., 2011; Quartana \& Burns, 2010). Prior research has demonstrated that engagement in reappraisal results in lower HR, DBP, negative emotions, and increased HRV and positive emotions when compared to control or suppression conditions (Butler et al., 2006; Denson, 2011; Quartana \& Burns, 2010; Roberts et al., 2008; vanOyen Witvliet et al., 2010). The results from the present study and previous research suggest that reappraisal produces positive cardiovascular benefits, particularly when confronting emotionally provocative films. However, in the present study we did not observe similar effects for HR, LF HRV, HF HRV, PA, or NA. The lack of an instruction effect on the other physiological and affective measures could have occurred as a result of the emotion (i.e., fear) that was induced, the coherence of the instructions (reappraisal and suppression) presented to participants, as well as the use of film clips (external procedure) rather than exposure to an actual fear-evoking event or stimulus. Further, the discordance between physiological measures and affective measures observed in the present study has also been demonstrated in previous research (Lang, Levin, Miller, \& Kozak, 1983). Therefore, it is possible that the lack of consistent findings across all dependent measures resulted from desynchrony between emotion self-report and physiological measures.

The present study differed from past research in several important ways, which could have contributed to the less than consistent results that were observed. In two prior studies (Butler et al., 2006; Roberts et al., 2008), the stressor task induced negative emotions by using a documentary film of World War II in the former and film clips involving a limb amputation and 
a burn victim in the latter. In contrast, the present study induced fear using two fear film clips from recent horror films. The induction of fear was successful, as evidenced by increased ratings of negative affect from the rest to task periods. Although the induction of NA was successful, there is evidence suggesting that the regulation of fear may differ from the regulation of other negative emotions (Webb, Miles, \& Sheeran, 2012). In reviewing the effects of emotion regulation, Webb et al. (2012) found differences between positive and negative emotions, as well as differences between what is called hedonic and contra-hedonic emotion regulation. Hedonic emotion regulation refers to attempts to improve affect or mood by either increasing positive affect or decreasing negative affect (e.g., seek pleasure, avoid pain). In contrast, contra-hedonic emotion regulation refers to attempts to worsen affect or mood by decreasing positive affect or increasing negative affect. In Webb’s study, contra-hedonic emotion regulation of positive emotions produced larger effect sizes than hedonic regulation of negative affect. In the present study, because participants engaged in hedonic regulation of fear, larger effects sizes would not be predicted based upon the findings of Webb and colleagues. Perhaps the hedonic regulation attempted in the current study produced small effect sizes, which were not observed among all of the dependent measures. Further, recent research (Verduyn, Delvaux, Van Coillie, Tuerlinckx, \& Van Mechelen, 2009) found differences in the temporal effects of various emotions.

Specifically, fear tended to have the shortest temporal effect as compared to sadness and anger. Sadness, which produced the longest temporal effects of negative emotions, had the largest effect size as compared to other negative emotions (Webb et al., 2012). Therefore, it is possible that temporal effects of emotions influenced the effects observed in the present study in which exposure to fear was used rather than exposure to anger or sadness. 
In addition to considering how the target emotion elicited in this study influenced results, it is also important to consider how the emotion regulation instructions employed in the study may have affected study outcomes. Webb et al. (2012) examined differences in types of reappraisal and suppression instructions as well as determining the effect sizes for reappraisal and suppression instructions. According to Webb et al., reappraisal had a small, but unreliable effect $(d=.05)$ on physiological measures. In contrast, reappraisal produced small-to-medium effects on self-report outcomes $(d=.45)$. In addition to determining effect sizes of reappraisal, Webb et al. (2012) identified four different types of reappraisal and found that effects of these strategies varied depending on the type of instructions presented. Another study by Butler et al. (2006) employed reappraisal of an emotional stimulus and found greater increases of HRV among reappraisal conditions when compared to suppression and instructions conditions. Unlike the study by Butler et al., the present study employed reappraisal via perspective taking, which was adapted from Gross (1998b). Reappraisal via perspective taking instructs participants to view an emotional stimulus in an objective or detached manner. Several prior studies that measured physiological reactivity when participants engaged in reappraisal via perspective taking (Ayduk \& Kross, 2008; Gross, 1998b; Low, Stanton, \& Bower, 2008; Ray, Wilhelm, \& Gross, 2008; Williams, Bargh, Nocera, \& Gray, 2009) produced mixed findings. In some of these studies, reappraisal via perspective taking did not produce decreased sympathetic activity when compared to suppression and control conditions (Gross, 1998b; Low et al., 2008; Ray et al., 2008). In contrast, one study found that participants who engaged in reappraisal via perspective taking demonstrated lower heart rate reactivity when compared to control conditions (Williams et al., 2009). Similarly, in yet another study, participants instructed to reappraise via perspective taking demonstrated lower mean arterial pressure (MAP) reactivity when compared 
to participants engaged in rumination (Ayduk \& Kross, 2008). These results suggest that the effects of a specific form of reappraisal, perspective taking, are inconsistent with respect to physiological reactivity to emotional provocation.

Unlike reappraisal, the effects of suppression in the literature are more consistent. Webb et al. (2012) determined that suppression produced a small, negative effect on physiological measures $(d=-.19)$, but no reliable effects on self-report measures of affect $(d=.03)$. Similar to examining differences in types of reappraisal instructions, Webb et al. examined differences in four types of suppression instructions. Suppressing the expression of an emotion, one type of suppression instruction, was used in the present study. Participants engaged in this type of suppression are instructed to behave in such a way that an observer would be unaware of the emotion being experienced (Webb et al., 2012). Multiple studies examined the effects of suppressing the expression of an emotion (Quartana \& Burns, 2007; Roberts et al. 2008) and demonstrated that this type of suppression typically produces increased sympathetic activation.

The lack of consistent findings and small effect sizes demonstrated for reappraisal and suppression in the literature (e.g., Webb et al., 2012) could explain the less than consistent findings in the present study. The main outcome measures used in this study consisted of five physiological measures (SBP, DBP, HR, LF HRV, and HF HRV), and as already discussed, reappraisal typically produces small-to-medium effect sizes on physiological measures (Webb et al., 2012). However, the effects of the specific form of reappraisal used in the current study, perspective taking, are less consistent. Similarly, suppression overall only produces small, negative effects on physiological measures.

Importantly, as noted in the method section, the power analysis conducted prior to this study determined that 56 participants were needed to detect large effects in the present study. 
However, due to the challenges in recruitment of participants, the sample of the current study only consisted of 33 participants. Consequently, it is possible that the study was not adequately powered to detect the smaller effects typically observed for physiological measures in studies employing reappraisal and suppression. Despite the lack of power, we observed a main effect of instructions for both SBP and DBP.

\section{Strengths and Limitations}

To better evaluate the findings of this study, it is important to consider the multiple strengths and limitations of the study. First, the design of the study, a mixed factors (within- and between-subjects) design, enabled us to examine the effects of both habitual and instructional use of emotion regulation strategies in a single study. Further, the design of the study enabled us to control for individual difference characteristics by requiring all participants to engage in both reappraisal and suppression. Controlling for individual difference characteristics is particularly important when examining physiological reactivity due to the many factors that influence one’s physiological reactivity profile. Second, this study employed multi-modal assessment of the fear response, including assessing affective, physiological, and behavioral responses. The ideal physiological measure of fear, skin conductance, was not employed in the present study because of a lack of equipment and as such represents one limitation of the study. However, the physiological measures used in the present study enabled examination of both sympathetic and parasympathetic activation, which have not been examined extensively in the emotion regulation literature. Further, although a few studies (Gross, 1998b; Gross, 1999; Gross \& Levenson, 1993; Roberts et al., 2008) have measured behavioral expressions of emotion, the behavioral measure used in the current study only focused on one behavioral element (e.g., eye contact). In fact, the primary goal for including measures of behavior in the present study was to ensure that 
participants did not engage in another emotion regulation strategy (i.e., attentional deployment). Although it served well in this capacity, it also functioned as a relatively crude measure of behavioral avoidance typically associated with a fear response. The design of the study and the use of multi-modal assessment of fear enabled the present study to examine the effects of emotion regulation strategies in a more comprehensive manner than some previous investigations.

Although this study had a strong design and comprehensive assessment of physiological, affective, and behavioral responses, there were several limitations that must be addressed. The first limitation relates to recruitment of participants. Incentives for participation were changed several times. Initially participants were offered one hour of extra credit and then a lottery was instituted. After the lottery was instituted, the amount that participants could potentially win was changed. Originally, it was planned that 10 participants from the lab sample would each win $\$ 25$; however, the amount of the lottery was increased to \$50 each to enhance recruitment. This change in incentive potentially could have resulted in differences in the type of participant who was willing to schedule a laboratory session. However, because of the small sample size (particularly those who were recruited after the change in incentive), we were unable to examine differences in participant characteristics based on the change in incentives. In addition to the change in incentives, eligibility criteria for participating in the lab phase of the study were changed. One previous study (Menchola, 2008) used the $75^{\text {th }}$ and $25^{\text {th }}$ percentiles on the subscales of the ERQ to classify participants as habitual reappraisers or suppressors. Although this was the original criteria proposed for the study, the criteria were expanded to enhance recruitment. Ultimately, eligible participants were identified as those scoring in the upper $66^{\text {th }}$ and lower $50^{\text {th }}$ percentiles on subscales of the ERQ. Adjusting the eligibility criteria was another 
limitation of the study as this resulted in less extreme groups on measures of emotion regulation. Because the groups were defined using these more liberal criteria, it is likely that the habitual emotion regulation groups in the current study did not represent high reappraisers/low suppressors and high suppressors/low reappraisers respectively. Indeed, comparisons of study means among groups in the current study were less extreme than those reported in previous studies (Mauss et al., 2007; Menchola, 2008). Despite the lack of correspondence between groups in the current and previous studies, it is important to note that no norms exist to identify habitual reappraisers and habitual suppressors. Therefore, it is possible that the eligibility criteria used in this study are adequate for identifying habitual reappraisers and suppressors. Additional research is necessary to create a normative approach for using the ERQ to identify habitual reappraisers and suppressors.

This lack of well-defined groups as well as the demographic characteristics of participants limits the generalizability of findings. Participants in this study were primarily Caucasian, female, and healthy (e.g., nonsmokers) young adults (college-aged students). Although there is an obvious concern regarding the generalizability of results, it is important to note that research has examined racial, gender, and age differences in emotion regulation and found that physiological effects of emotion regulation are not related to these variables. For example, previous research demonstrated consistent physiological responses to suppression among four different ethnic groups (African American, Chinese American, European American, and Mexican American). Similarly, research has demonstrated no age differences between older$\left(\mathrm{M}_{\mathrm{age}}=71\right.$ years $)$ and younger-adults $\left(\mathrm{M}_{\mathrm{age}}=21\right.$ years $)$ in physiological responses during engagement in suppression (Kunzmann, Kupperbusch, \& Levenson, 2005). Further, this study also demonstrated no gender differences in physiological responses (Kunzmann et al., 2005). 
Although there may be some age differences in strategy preference (e.g., older adults prefer situation selection) and gender differences in emotion regulation in interpersonal situations, research demonstrated that the effects of emotion regulation upon physiological responses to emotion provocation are consistent across race, gender, and age (Charles \& Carstensen, 2008; Kunzmann et al., 2005). Finally, it was necessary to have strict exclusionary criteria to examine the effects of the experimental manipulation free from potential confounds. For example, in research examining physiological responses, smokers are typically excluded as the physiological effects of nicotine withdrawal could confound results. Although the exclusionary criteria may further limit generalizability, it was critical for the design of the study.

Additional limitations could explain the lack of findings in the present study and are important methodological concerns that future research should examine. Several preliminary analyses were conducted to examine differences in physiological and affective reactivity between the neutral film clips and fear films as well as examining differences in reactivity between the rest periods (pre-reappraisal and pre-suppression) and task periods (reappraisal and suppression). In examining the differences in reactivity between the two neutral film clips and the two fear film clips, two differences in physiological reactivity were found. For the neutral film clips, LF HRV was lower during neutral film I as compared to LF HRV during neutral film II. This suggests that the neutral film I resulted in somewhat greater relaxation, as compared to neutral film II, which suggests that there were differences in the level of parasympathetic and sympathetic activation as well as differences in the level of the target emotion evoked by the neutral clips. However, previous research (Kolodyazhniy et al., 2011; Kreibig et al., 2004; Kreibig et al., 2007) did not include measures of parasympathetic activation, which could explain why these differences were not observed. In addition, for the fear films, SBP was higher during 
fear film clip II as compared to fear film clip I, indicating that the second film clip evoked greater levels of fear (the target emotion). Future research should explore if these findings are reliable and reflect subtle differences between the two film clips and their ability to elicit physiological and affective reactivity. Researchers considering using these films sets must be aware of these differences and carefully consider the use of these film stimuli in studies.

An additional concern regarding the use of these film clips is whether these particular fear film clips evoked sufficient levels of physiological and affective reactivity. In examining reactivity from the neutral film clip rest periods (pre-reappraisal and pre-suppression) to reactivity during the fear film clip task periods (reappraisal and suppression), there was limited evidence of physiological and affective reactivity. Only, SBP, DBP, and NA demonstrated reactivity from the neutral film clip rest periods to the fear film clip task periods. Therefore, it is possible that the limited findings in the study resulted from the relative lack of physiological and affective reactivity elicited by these two film clips. Further, recent research (Salas, Radovic, \& Turnbull, 2011) has demonstrated differences in exposure to external stimuli (viewing film clips) as opposed to exposure to internal stimuli (recalling personal memories). Specifically, exposure to external stimuli evokes less negative emotion than exposure to internal stimuli (Salas et al., 2011). Therefore, it is possible that the film clips used in this study were insufficient in producing physiological and affective reactivity. For researchers, it is important to consider how differences in emotion regulation may relate to whether emotion was elicited via external or internal cues.

The final two limitations of the current study are sample size and the order of clip presentation. Originally, we proposed a sample size of 56 participants, which was based upon a power analysis to detect large effects and having an equal number of participants in each 
condition. However, because of recruitment difficulties, data was only collected on 33

participants. As a result, the study was underpowered, which could partially explain the lack of consistent findings. One additional limitation that should be noted is that the instructional sets presented to participants were not completely counterbalanced across film sets. The film clips used in the study consisted of Set A (Neutral Film I and Fear Film I) and Set B (Neutral Film II and Fear Film II). The order of film set presentation was counterbalanced within groups (e.g., half of habitual reappraisers received Set A first and half received Set B first) and the order of instructions was counterbalanced within groups (e.g., half of habitual reappraisers received instructions to reappraise first and half received instructions to suppress first). However, the instructional sets (reappraisal vs. suppression) were not counterbalanced across film sets. Specifically, for habitual reappraisers, the instructional set to reappraise was always paired with film Set A, and the instructional set to suppress was always paired with film Set B. Similarly, for habitual suppressors, the instructional set to suppress was always paired with film Set A, and the instructional set to reappraise was always paired with film Set B. If the instructional sets were completely counterbalanced across film sets, eight conditions would have been required. To address the fact that instructional sets were not counterbalanced across film sets, we conducted several paired sample t-tests to compare physiological and affective reactivity on the neutral film clips and the fear film clips respectively. As discussed previously, there was evidence of greater parasympathetic activation on neutral film I as compared to neutral film II and greater sympathetic activation on fear film II as compared to fear film I. Because of the potential differences in sympathetic activity between fear films and the fact that the instructional sets were not counterbalanced across film sets, it is possible that effects observed in this study are unique to a specific film set. 


\section{Future Directions and Conclusions}

Although there has been a sharp increase in research examining emotion regulation in recent years, there are critical gaps remaining in the literature. One of the most critical directions for future research in emotion regulation is determining if emotion regulation effects on physiological responding are emotion specific. Specifically, are there physiological, affective, or behavioral differences in the effects of reappraisal and suppression for different emotions? For example, are the effects of reappraisal and suppression similar across other negative emotions (e.g., anger or sadness) as well as positive emotions (e.g., happiness or amusement)? Researchers will need to examine emotion regulation differences for both positive and negative emotions in future research.

Understanding emotion regulation naturally leads to discussions of how training in emotion regulation may be associated with improved psychological and physical health. In this regard, future research should examine the effects of clinical interventions incorporating emotion regulation as well as the generalizability and long-term effects of emotion regulation. Despite not observing an interaction effect of habitual-strategy use by instructional set or a main effect for habitual strategy, we maintain that examining the effects of habitual-strategy use in emotion regulation is critical. It is important to note that as a result of the lack of power in the current study, we were unable to conclude that there was no effect of habitual-strategy use. It is possible that habitual-strategy use produces effects on physiological and affective measures that were not detected in the present study. As clinical interventions incorporating emotion regulation strategies are currently being developed, it is important to determine if the effectiveness of such interventions are influenced by one’s habitual-strategy use (Finkel, Slotter, Luchies, Walton, \& Gross, 2013). Research focusing on the development and testing of clinical interventions based 
on emotion regulation is one future direction; however, it is also critical that emotion regulation research examine the long-term benefits or consequences of reappraisal and suppression and the generalizability of emotion regulation effects. Clearly, reappraisal produces short-term benefits for cardiovascular responses (Wolgast el., 2011); however, it is not presently known if these benefits are maintained. Further, future research should examine the generalizability of reappraisal and suppression to natural settings. It is critical to examine if engagement in reappraisal produces the same effects, larger effects, or smaller effects following a natural stressor (e.g., thesis defense meeting). Although research has demonstrated that reappraisal produces less sympathetic activation than suppression (Butler et al., 2003; Denson et al., 2011), it is necessary to also demonstrate if the effects of reappraisal are generalizable to other stressors and if the effects are maintained. It is also possible that while habitual emotion regulation strategy did not influence one's acute response to emotion provocation, it is entirely possible that habitual emotion regulation strategy does influence one’s response to naturally-occurring stressors. Only future research in this area will bear this out.

In conclusion, this study represents one of only two studies (Menchola, 2008) to examine the effects of habitual and instructional emotion regulation strategies. Unlike other studies (Denson et al, 2011; Menchola, 2008; Quartana \& Burns, 2010; Roberts et al. 2008), the present study included comprehensive measures of affective, physiological, and behavioral responses. Critically, this study supports previous research in demonstrating that reappraisal produces decreases in sympathetic activation (Wolgast et al., 2011) when compared with suppression. Based on the results of this study, which are tempered by several limitations, the effects of emotion regulation strategy are evident when participants were instructed to engage in specific 
strategies. The role of habitual emotion regulation strategy in response to emotion provocation will require additional research on larger and more clearly defined samples. 


\section{References}

Aldao, A., \& Mennin, D. S. (2012). Paradoxical cardiovascular effects of implementing adaptive emotion regulation strategies in generalized anxiety disorder. Behaviour Research and Therapy, 50(2), 122-130. doi:10.1016/j.brat.2011.12.004

Aldao, A., Nolen-Hoeksema, S., \& Schweizer, S. (2010). Emotion-regulation strategies across psychopathology: A meta-analytic review. Clinical Psychology Review, 30(2), 217-237. doi:10.1016/j.cpr.2009.11.004

Antony, M. M. (2001). Measures for specific phobia. In M. M. Antony, S. M., Orsillo, \& L. Roemer (Eds). Practitioner's guide to empirically based measures of anxiety. (pp. 145146). New York, NY US: Kluwer Academic/Plenum Publishers.

Ayduk, O., \& Kross, E. (2008). Enhancing the pace of recovery: Self-distanced analysis of negative experiences reduces blood pressure reactivity. Psychological Science, 19, 229 -231. doi: doi:10.1111/j.1467-9280.2008.02073.x

Beasley, W., Chaffin, S., Feig, E., Moritz, N.H., (Producers) \& Cannon, D. (Director) (1998). I still know what you did last summer [Motion picture]. Culver City, CA: Columbia Tristar Home Video.

Berntson, G. G., Uchino, B. N., \& Cacioppo, J. T. (1994). Origins of baseline variance and the law of initial values. Psychophysiology, 31(2), 204-210. doi:10.1111/j.1469 8986.1994.tb01042.x

Berntson, G. G., Bigger Jr., T., Eckberg, D. L., Grossman, P., Kaufman, P. G., Malik, M.,..Van Der Molen, M.W. (1997). Heart rate variability: Origins, methods, and interpretive caveats. Psychophysiology, 34, 623-648. 
Butler, E. A., Egloff, B., Wilhelm, F. H., Smith, N. C., Erickson, E. A., \& Gross, J. J. (2003).The social consequences of expressive suppression. Emotion, 3(1), 48-67. doi: 10.1037/1528 3542.3.1.48

Butler, E. A., Wilhelm, F. H., \& Gross, J. J. (2006). Respiratory sinus arrhythmia, emotion, and emotion regulation during social interaction. Psychophysiology, 43(6), 612-622. doi:10.1111/j.1469-8986.2006.00467.x

Cannon, W. B. (1927). The James-Lange theory of emotions: A critical examination and an alternative theory. The American Journal of Psychology, 39(1), 106-124.

Chaffin, S., Feig, E., Moritz, N. H., (Producers) \& Gillespie, J. (Director) (1997). I know what you did last summer [Motion picture]. Culver City, CA: Columbia Tristar Home Video.

Charles, S. T., \& Carstensen, L. L. (2008). Unpleasant situations elicit different emotional responses in younger and older adults. Psychology and Aging, 23(3), 495-504. doi: 10.1037/a0013284

Cisler, J. M., Olatunji, B. O., Feldner, M. T., \& Forsyth, J. P. (2010). Emotion regulation and the anxiety disorders: An integrative review. Journal of Psychopathology and Behavioral Assessment, 32(1), 68-82. doi:10.1007/s10862-009-9161-1

Cole, P. M., Martin, S. E., \& Dennis, T. A. (2004). Emotion regulation as a scientific construct: Methodological challenges and directions for child development research. Child Development, 75(2), 317-333. doi: 0009-3920/2004/7502-0002

Crawford, J. R., \& Henry, J. D. (2004). The positive and negative affect schedule (PANAS): Construct validity, measurement properties and normative data in a large non-clinical sample. British Journal of Clinical Psychology, 43(3), 245-265. doi:10.1348/0144665031752934 
Dan-Glauser, E. S., \& Gross, J. J. (2011). The temporal dynamics of two response-focused forms of emotion regulation: Experiential, expressive, and autonomic consequences. Psychophysiology, 48(9), 1309-1322. doi:10.1111/j.1469-8986.2011.01191.x

Davis, P. J., \& Schwartz, G. E. (1987). Repression and the inaccessibility of affective memories. Journal of Personality and Social Psychology, 52(1), 155-162. doi:10.1037/00223514.52.1.155

Denson, T. F., Grisham, J. R., \& Moulds, M. L. (2011). Cognitive reappraisal increases heart rate variability in response to an anger provocation. Motivation and Emotion, 35(1), 14-22. doi:10.1007/s11031-011-9201-5

Dickson, K. S., Ciesla, J. A., \& Reilly, L. C. (2011). Rumination, worry, cognitive avoidance, and behavioral avoidance: Examination of temporal effects. Behavior Therapy, doi:10.1016/j.beth.2011.11.002

Dunn, B. D., Billotti, D., Murphy, V., \& Dalgleish, T. (2009). The consequences of effortful emotion regulation when processing distressing material: A comparison of suppression and acceptance. Behaviour Research and Therapy, 47(9), 761-773. doi:10.1016/j.brat.2009.05.007

Faul, F., Erdfelder, E., Buchner, A., \& Lang, A.G. (2009). Statistical power analyses using G*Power 3.1: Tests for correlation and regression analyses. Behavior Research Methods, 41, 1149-1160. doi: 10.3758/BRM.41.4.1149

Field, A. (2013). Discovering statistics using IBM SPSS statistics. Fourth Ed. Los Angeles, California: Sage Publishers. 
Finkel, E. J., Slotter, E. B., Luchies, L. B., Walton, G. M., Gross, J. J. (2013). A brief intervention to promote conflict reappraisal preserves marital quality over time. Psychological Science. Advance online publication. doi: 10.1177/0956797612474938

Fredrickson, B. L., \& Levenson, R. W. (1998). Positive emotions speed recovery from the cardiovascular sequelae of negative emotions. Cognition and Emotion, 12(2), 191-220. doi:10.1080/026999398379718

Gabbay, F. H., Krantz, D. S., Kop, W. J., Hedges, S. M., Klein, J., Gottdiener, J. S., \& Rozanski, A. (1997). Triggers of myocardial ischemia during daily life in patients with coronary artery disease: Physical and mental activities, anger and smoking. Journal of the American College of Cardiology, 27, 585-592.

Gallo, L. C., Ghaed, S. G., \& Bracken, W. S. (2004). Emotions and cognitions in coronary heart disease: Risk, resilience, and social context. Cognitive Therapy and Research, 28(5), 669 694. doi:10.1023/B:COTR.0000045571.11566.19

Goodie, J. L., Larkin, K. T., \& Schauss, S. (2000). Validation of Polar heart rate monitor for assessing heart rate during physical and mental stress. Journal of Psychophysiology, 14(3), 159-164. doi: 10.1027//0269-8803.14.3.159

Gross, J. J. (1998a). The emerging field of emotion regulation: An integrative review. Review of General Psychology, 2(3), 271-299. doi:10.1037/1089-2680.2.3.271

Gross, J. J. (1998b). Antecedent- and response-focused emotion regulation: Divergent consequences for experience, expression, and physiology. Journal of Personality and Social Psychology, 74(1), 224-237. doi:10.1037/0022-3514.74.1.224

Gross, J. J. (1999). Emotion regulation: Past, present, future. Cognition and Emotion, 13(5), 551573. doi:10.1080/026999399379186 
Gross, J. J., \& John, O. P. (2003). Individual differences in two emotion regulation processes: Implications for affect, relationships, and well-being. Journal of Personality and Social Psychology, 85(2), 348-362. doi:10.1037/0022-3514.85.2.348

Gross, J. J., \& Levenson, R. W. (1993). Emotional suppression: Physiology, self-report, and expressive behavior. Journal of Personality and Social Psychology, 64(6), 970-986. doi:10.1037/0022-3514.64.6.970

Gross, J. J., \& Levenson, R. W. (1995). Emotion elicitation using films. Cognition and Emotion, 9(1), 87-108. doi:10.1080/02699939508408966

Gross, J. J., \& Levenson, R. W. (1997). Hiding feelings: The acute effects of inhibiting negative and positive emotion. Journal of Abnormal Psychology, 106(1), 95-103. doi:10.1037/0021-843X.106.1.95

Gross, J. J. \& Thompson, R.A. (2007). Emotion regulation: Conceptual foundations. In J. J. Gross, \& J. J. Gross (Eds.), Handbook of emotion regulation. (pp. 3-20). New York, NY US: Guilford Press.

Gullette, E.C., Blumenthal, J.A., Babyak, M., Jiang, W., Waugh, R.A., Frid, D.J., ... Krantz, D.S. (1997). Effects of mental stress on myocardial ischemia during daily life. JAMA, 277, 1521-1526.

Hofmann, S. G., Heering, S., Sawyer, A. T., \& Asnaani, A. (2009). How to handle anxiety: The effects of reappraisal, acceptance, and suppression strategies on anxious arousal. Behaviour Research and Therapy, 47(5), 389-394. doi:10.1016/j.brat.2009.02.010

Jamieson, J. P., Nock, M. K., \& Mendes, W. B. (2012). Mind over matter: Reappraising arousal improves cardiovascular and cognitive responses to stress. Journal of Experimental Psychology: General, 141(3), 417-422. doi:10.1037/a0025719 
James, W. (1884). What is an emotion? Mind, 9, 188-205. Retrieved from http://psychclassics.yorku.ca/James/emotion.htm

John, O. P., \& Gross, J. J. (2004). Healthy and unhealthy emotion regulation: Personality processes, individual differences, and life span development. Journal of Personality, 72(6), 1301-1333. doi:10.1111/j.1467-6494.2004.00298.x

John, O. P., \& Gross, J. J. (2007). Individual differences in emotion regulation. In J. J. Gross, \& J. J. Gross (Eds.), Handbook of emotion regulation. (pp. 351-372). New York, NY US: Guilford Press.

Just, N., \& Alloy, L. B. (1997). The response styles theory of depression: Tests and an extension of the theory. Journal of Abnormal Psychology, 106(2), 221-229. doi:10.1037/0021843X.106.2.221

Kolodyazhniy, V., Kreibig, S. D., Gross, J. J., Roth, W. T., \& Wilhelm, F. H. (2011). An affective computing approach to physiological emotion specificity: Toward subject independent and stimulus-independent classification of film-induced emotions. Psychophysiology, 48(7), 908-922. doi:10.1111/j.1469-8986.2010.01170.x

Kreibig, S.D., Ferstl, R., \& Roth, W.T. (2004). Situational and individual response specificity to emotional films: Effects on experiential, cardiovascular, electrodermal, respiratory, and muscular responses. (Master’s Thesis Institute of Psychology, Christian-Albrechts University, Kiel, Germany). Retrieved from Google, http://www.affective sciences.org/system/files/Kreibig\%2C\%202004\%20\%28diploma\%20thesis\%29.pdf

Kreibig, S. D., Wilhelm, F. H., Roth, W. T., \& Gross, J. J. (2007). Cardiovascular, electrodermal, and respiratory response patterns to fear- and sadness-inducing films. Psychophysiology, 44(5), 787-806. doi:10.1111/j.1469-8986.2007.00550.x 
Kubzansky, L. D., \& Kawachi, I. (2000). Going to the heart of the matter: Do negative emotions cause coronary heart disease? Journal of Psychosomatic Research, 48(4-5), 323-337. doi:10.1016/S0022-3999(99)00091-4

Kunzmann, U., Kupperbusch, C. S., \& Levenson, R. W. (2005). Behavioral inhibition and amplification during emotional arousal: A comparison of two age groups. Psychology and Aging, 20(1), 144-158. doi:10.1037/0882-7974.20.1.144

Lang, P. J. (1995). The emotion probe: Studies of motivation and attention. American Psychologist, 50(5), 372-385. doi:10.1037/0003-066X.50.5.372

Lang, P. J., Levin, D. N., Miller, G. A., \& Kozak, M. J. (1983). Fear behavior, fear imagery, and the psychophysiology of emotion: The problem of affective response integration. Journal of Abnormal Psychology, 92(3), 276-306.

Larkin, K. T. (2005). Stress and hypertension: Examining the relation between psychological stress and high blood pressure. New Haven, CT: Yale University Press.

Low, C. A., Stanton, A. L., \& Bower, J. E. (2008). Effects of acceptance-oriented versus evaluation emotional processing on heart rate recovery and habituation. Emotion, 8, 419 -424. doi:10.1037/1528-3542.8.3.419

Marler, M. R., Jacob, R. G., Lehoszky, J. P., \& Shapiro, A. P. (1988). The statistical analysis of treatment effects in 24-hour ambulatory blood pressure recordings. Statistics in Medicine, 7, 697-716.

Mauss, I. B., Cook, C. L., Cheng, J. Y. J., \& Gross, J. J. (2007). Individual differences in cognitive reappraisal: Experiential and physiological responses to an anger provocation. International Journal of Psychophysiology, 66(2), 116-124. doi:10.1016/j.ijpsycho.2007.03.017 
McCubbin, J. A., Merritt, M. M., Sollers III, J. J., Evans, M. K., Zonderman, A. B., Lane, R. D., \& Thayer, J. F. (2011). Cardiovascular-emotional dampening: The relationship between blood pressure and recognition of emotion. Psychosomatic Medicine, 73(9), 743-750. doi:10.1097/PSY.0b013e318235ed55

McCubbin, J.A., Surwit, R.S., \& Williams, R.B. (1985). Endogenous opiate peptides, stress reactivity, and risk for hypertension. Hypertension, 7, 808-811. doi: 10.1161/01.HYP.7.5.808

McRae, K., \& Gross, J. J. (2009). Emotion regulation. In D. Sander \& K. Scherer (Eds.), Oxford companion to the affective sciences. New York, NY: Oxford University Press.

McRae, K., Jacobs, S. E., Ray, R. D., John, O. P., \& Gross, J. J. (2012). Individual differences in reappraisal ability: Links to reappraisal frequency, well-being, and cognitive control. Journal of Research in Personality, doi:10.1016/j.jrp.2011.10.003

Melanko, S. (2011). The relation of delay discounting to health behavior. (Master's Thesis). Retrieved from WVU Scholar, West Virginia University, Morgantown, WV.

Menchola, M. (2008). Physiological measures of affective chronometry during habitual and voluntary use of emotion regulation strategies. (Doctoral Dissertation, The University of Arizona). Retrieved from Google Scholar, http://arizona.openrepository.com/arizona/bitstream/10150/194046/1/azu_etd_2392_sip _m.pdf

Mohan, A., Sharma, R., \& Bijlani, R. L. (2011). Effect of meditation on stress-induced changes in cognitive functions. The Journal of Alternative and Complementary Medicine, 17(3), 207-212. doi:10.1089/acm.2010.0142 
Moore, S. A., Zoellner, L. A., \& Mollenholt, N. (2008). Are expressive suppression and cognitive reappraisal associated with stress-related symptoms? Behaviour Research and Therapy, 46(9), 993-1000. doi:10.1016/j.brat.2008.05.001

Nelson, K. S., Weber, R. R., Elliott, W. J., Zebrauskas, D., \& Murphy, M. B. (1988). Evaluation of the Suntech Accutracker. A novel noninvasive ambulatory blood pressure monitor. American Journal of Hypertension, 1, 121S-123S.

Niskanen, J., Tarvainen, M. P., Ranta-aho, P. O., \& Karjalainen, P. A. (2002). Software for advances HRV analysis. Submitted to Computer Methods and Programs in Biomedicine.

Nyklíček, I., Vingerhoets, A. J. J. M., \& Van Heck, G. L. (1996). Hypertension and objective and self-reported stressor exposure: A review. Journal of Psychosomatic Research, 40(6), 585-601. doi:10.1016/0022-3999(95)00647-8

Prentice, P. R. (2009). Dispositional mindfulness and cardiovascular reactivity to sensory intake and sensory rejection tasks. (Master’s Thesis). Retrieved from WVU Scholar, West Virginia University, Morgantown, WV.

Quartana, P. J., \& Burns, J. W. (2010). Emotion suppression affects cardiovascular responses to initial and subsequent laboratory stressors. British Journal of Health Psychology, 15(3), 511-528. doi:10.1348/135910709X474613

Rapaport, D. (1953). On the psycho-analytic theory of affects. International Journal of Psychoanalysis, 34, 177-198.

Ray, R. D., Wilhelm, F. H., \& Gross, J. J. (2008). All in the mind's eye? Anger rumination and reappraisal. Journal of Personality and Social Psychology, 94 133-145. doi: 10.1037/0022-3514.94.1.133 
Roberts, N. A., Levenson, R. W., \& Gross, J. J. (2008). Cardiovascular costs of emotion suppression cross ethnic lines. International Journal of Psychophysiology, 70(1), 82-87. doi:10.1016/j.ijpsycho.2008.06.003

Rohlfing, B. (Director) (1997). Alaska's wild Denali [Motion picture]. Anchorage, AK: Alaska Video Postcards Inc.

Salas, C. E., Radovic, D., \& Turnbull, O. H. (2012). Inside-out: Comparing internally generated and externally generated basic emotions. Emotion, 12(3), 568-578. doi: $10.1037 / \mathrm{a} 0025811$

Sapolsky, R. M. (2007). Stress, stress-related disease, and emotional regulation. In J. J. Gross, \& J. J. Gross (Eds.), Handbook of emotion regulation. (pp. 606-615). New York, NY US: Guilford Press.

Sapolsky, R., Romero, M., \& Munck, A. (2000). How do glucocorticoids influence the stress response?: Integrating permissive, suppressive, stimulatory, and preparative actions. Endocrinology Review, 21, 55-89.

Schachter, S. \& Singer, J.E. (1962). Cognitive, social, and physiological determinants of emotional state. Psychological Review, 69(5), 379-399.

Sheppes, G., Scheibe, S., Suri, G., \& Gross, J. J. (2011). Emotion-Regulation Choice. Psychological Science, 22, 1391-1396. doi: 10.1177/0956797611418350

Sherwood, G. E., Allen, M. T., Fahrenberg, J., Kelsey, R. M., Lovallo, W. R., \& Van Doornen, L. J. P. (1990). Methodological guidelines for impedance cardiography. Psychophysiology, 27, 1-23.

Sirois, B. C., \& Burg, M. M. (2003). Negative emotion and coronary heart disease: A review. Behavior Modification, 27(1), 83-102. doi:10.1177/0145445502238695 
Stemmler, G. (1997). Selective activation of traits: Boundary conditions for the activation of anger. Personality and Individual Differences, 22(2), 213-233. doi:10.1016/S01918869(96)00189-4

Tabachnick, B.G. \& Fidell, L.S. (2012). Using multivariate statistics. Sixth Ed. Pearson.

Tarainen, M. P., \& Niskanen, J. P. (2008). Kubios HRV: User’s guide. Biosignal Analysis and Medical Imaging Group (BSAMIG). Retrieved from http://kubios.uku.fi/media/Kubios_HRV_Users_Guide.pdf

Ulrich-Lai, Y. M., \& Herman, J. P. (2009). Neural regulation of endocrine and autonomic stress responses. Nature Reviews Neuroscience, 10(6), 397-409. doi:10.1038/nrn2647

vanOyen Witvliet, C., Knoll, R. W., Hinman, N. G., \& DeYoung, P. A. (2010). Compassion focused reappraisal, benefit-focused reappraisal, and rumination after an interpersonal offense: Emotion-regulation implications for subjective emotion, linguistic responses, and physiology. The Journal of Positive Psychology, 5(3), 226-242.

doi:10.1080/17439761003790997

Vedhara, K., Hyde, J., Gilchrist, I. D., Tytherleigh, M., \& Plummer, S. (2000). Acute stress, memory, attention and cortisol. Psychoneuroendocrinology, 25(6), 535-549. doi:10.1016/S0306-4530(00)00008-1

Verduyn, P., Delvaux, E., Van Coillie, H. Tuerlinckx, F., \& Van Mechelen, I. (2009). Predicting the duration of emotional experience: Two experience sampling studies. Emotion, 9, 83 -91. doi: doi:10.1037/a0014610

Wang, M., \& Saudino, K. J. (2011). Emotion regulation and stress. Journal of Adult Development, 18(2), 95-103. doi:10.1007/s10804-010-9114-7 
Watson, D., Clark, L. A., \& Tellegen, A. (1988). Development and validation of brief measures of positive and negative affect: The PANAS scales. Journal of Personality and Social Psychology, 54(6), 1063-1070. doi:10.1037/0022-3514.54.6.1063

Webb, T. L., Miles, E., \& Sheeran, P. (2012). Dealing with feeling: A meta-analysis of the effectiveness of strategies derived from the process model of emotion regulation. Psychological Bulletin, 138(4), p. 775-808. doi: 10.1037/a0027600

Williams, L. E., Bargh, J. A., Nocera, C. C., \& Gray, J. R. (2009). The unconscious regulation of emotion: Nonconscious reappraisal goals modulate emotional reactivity. Emotion, 9, 847 -854. I doi:10.1037/a0017745

Wolgast, M., Lundh, L., \& Viborg, G. (2011). Cognitive reappraisal and acceptance: An experimental comparison of two emotion regulation strategies. Behaviour Research and Therapy, 49(12), 858-866. doi:10.1016/j.brat.2011.09.011 
Table 1

Main Demographic Characteristics of SONA (Screening) Sample and Lab Sample

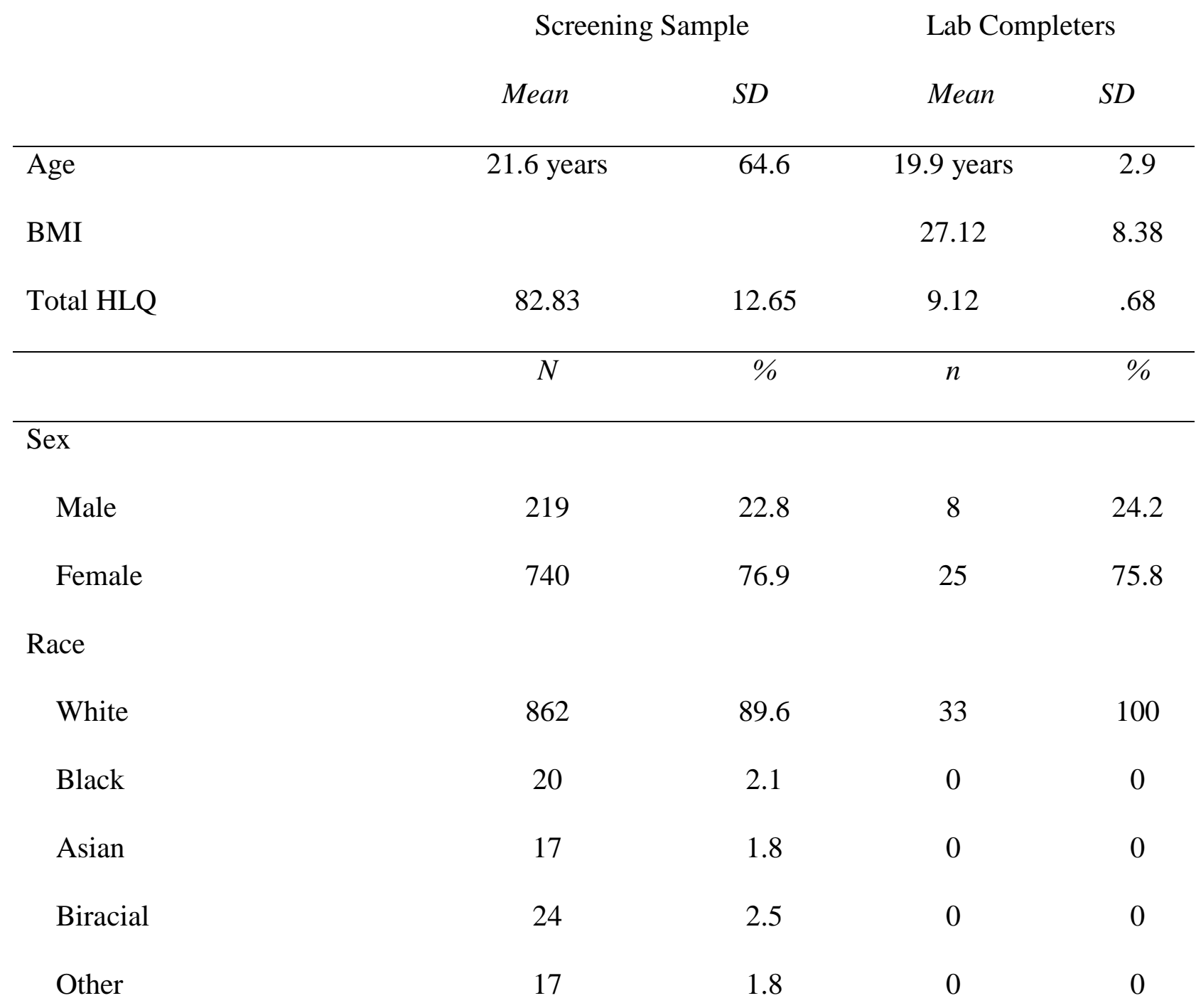

Ethnicity

Hispanic or Latino

29

3.0

100

Not Hispanic or Latino

907

94.3

32 0

*Note. Table includes demographic characteristics and means and standard deviations for variables collected thru SONA and for participants who participated in the lab phase. Total HLQ refers to the total score on the Healthy Lifestyle Questionnaire. 
Table 2

Additional Demographic Characteristics of SONA (Screening) Sample

Screening Sample

n $\quad \%$

Family Income

Less than $\$ 24,999$

$70 \quad 7.3$

$\$ 25,000$ to $\$ 34,9999$

$68 \quad 7.1$

$\$ 35,000$ to $\$ 49,999$

112

11.6

$\$ 50,000$ to $\$ 74,999$

175

18.2

$\$ 100,000$ to $\$ 149,999$

190

19.8

Greater than $\$ 150,000$

116

12.1

Father's Hx of Hypertension

Positive Hx

319

33.2

Negative Hx

612

63.6

Mother's Hx of Hypertension

Positive Hx

227

23.6

Negative Hx

719

74.7

Education

High School

310

32.2

1 year college

291

30.2

2 years college

190

19.8

3 years college

126

13.1

42

4.4

*Note. Table includes demographic characteristics and means and standard deviations for variables collected thru SONA. 
Table 3

Preliminary Analyses of Resting Measures of SBP and DBP

\begin{tabular}{lllll}
\hline & Mean & $S D$ & $F$ & $p$ \\
\hline SBP Minute 0 & $121.26^{\mathrm{a}}$ & 17.76 & & \\
SBP Minute 3 & $116.89^{\mathrm{a}}$ & 15.93 & & \\
SBP Minute 6 & 118.04 & 16.32 & & .03 \\
SBP Minute 9 & 117.44 & 17.18 & & \\
SBP Rest Period 1 & & & & \\
\hline DBP Minute 0 & 64.93 & 8.33 & & \\
DBP Minute 3 & 64.93 & 7.97 & & \\
DBP Minute 6 & 64.07 & 7.69 & & \\
DBP Minute 9 & 64.96 & 10.08 & & \\
DBP Rest Period 1 & & & & \\
\hline
\end{tabular}

Note. One-way repeated measures ANOVA. SBP and DBP (untransformed) are compared across recordings made during the first rest period. SBP and DBP are in mm Hg.

${ }^{a}$ means are significantly different $(p<.05)$ 
Table 4

Differences in Cardiovascular and Affective Reactivity between Neutral Film Clips

\begin{tabular}{|c|c|c|c|c|}
\hline & Mean & $S D$ & $t(d f)$ & $P$ \\
\hline SBP (mm Hg) & & & $\begin{array}{l}-.77(26) \\
\end{array}$ & .45 \\
\hline SBP N1 & 117.58 & 18.10 & & \\
\hline SBP N2 & 118.93 & 15.26 & & \\
\hline DBP (mm Hg) & & & $-1.34(26)$ & .18 \\
\hline DBP N1 & 77.44 & 9.64 & & \\
\hline DBP N2 & 79.08 & 9.37 & & \\
\hline HR (bpm) & & & $.15(31)$ & .87 \\
\hline HR N1 & 72.30 & 9.87 & & \\
\hline HR N2 & 71.99 & 14.85 & & \\
\hline LF HRV (Hz) & & & $-2.35(29)$ & .03 \\
\hline LF HRV N1 & 1642.37 & 1256.40 & & \\
\hline LF HRV N2 & 2936.42 & 2741.54 & & \\
\hline HF HRV (Hz) & & & $-.36(29)$ & .72 \\
\hline HF HRV N1 & 1879.76 & 2415.91 & & \\
\hline HF HRV N2 & 2004.89 & 1716.77 & & \\
\hline Positive Affect & & & $-.62(32)$ & .63 \\
\hline PA N1 & 20.09 & 7.78 & & \\
\hline PA N2 & 20.48 & 8.24 & & \\
\hline Negative Affect & & & $.49(32)$ & .63 \\
\hline NA N1 & 10.55 & 1.09 & & \\
\hline NA N2 & 10.45 & 1.09 & & \\
\hline
\end{tabular}

Note. N1 = Neutral film clip I; N2 = Neutral film clip II. All means are untransformed. 
Table 5

Differences in Cardiovascular and Affective Reactivity between Fear Film Clips

\begin{tabular}{|c|c|c|c|c|}
\hline & Mean & $S D$ & $t(d f)$ & $p$ \\
\hline SBP (mm Hg) & & & $-2.84(28)$ & .008 \\
\hline SBP F1 & 119.02 & 14.92 & & \\
\hline SBP F2 & 122.32 & 13.84 & & \\
\hline DBP (mm Hg) & & & $-1.74(28)$ & .09 \\
\hline DBP F1 & 80.11 & 8.43 & & \\
\hline DBP F2 & 81.61 & 8.66 & & \\
\hline HR (bpm) & & & $.43(30)$ & .67 \\
\hline HR F1 & 71.81 & 9.55 & & \\
\hline HR F2 & 70.91 & 16.09 & & \\
\hline LF HRV (Hz) & & & $.85(30)$ & .40 \\
\hline LF HRV F1 & 1921.87 & 2111.93 & & \\
\hline LF HRV F2 & 1582.10 & 1251.32 & & \\
\hline HF HRV (Hz) & & & $1.79(30)$ & .08 \\
\hline HF HRV F1 & 1839.68 & 1955.21 & & \\
\hline HF HRV F2 & 1424.44 & 1188.43 & & \\
\hline Positive Affect & & & $-1.01(31)$ & .32 \\
\hline PA F1 & 19.94 & 4.79 & & \\
\hline PA F2 & 20.44 & 4.89 & & \\
\hline Negative Affect & & & $.34(31)$ & .73 \\
\hline NA F1 & 17.25 & 5.71 & & \\
\hline NA F2 & 17.06 & 6.90 & & \\
\hline
\end{tabular}

Note. F1 = fear film clip I, F2 = fear film clip II. All means are untransformed. 
Table 6

Means and Standard Deviations for Covariates during Resting (Neutral Film) Periods

\begin{tabular}{|c|c|c|c|c|}
\hline & $\begin{array}{l}\text { Mean } \\
\text { (untransformed) }\end{array}$ & $\begin{array}{l}\text { SD } \\
\text { (untransformed) }\end{array}$ & $\begin{array}{l}\text { Mean } \\
\text { (transformed) }\end{array}$ & $\begin{array}{l}\text { SD } \\
\text { (transformed) }\end{array}$ \\
\hline Pre-Reappraisal SBP & 118.64 & 17.98 & 10.86 & .80 \\
\hline Pre-Reappraisal DBP & 78.88 & 10.30 & 8.86 & .58 \\
\hline Pre-Reappraisal HR & 73.37 & 13.44 & & \\
\hline Pre-Reappraisal LF HRV & 2501.41 & 2243.28 & 3.21 & .44 \\
\hline Pre-Reappraisal HF HRV & 1784.40 & 1576.36 & 3.06 & .46 \\
\hline Pre-Reappraisal PA & 20.21 & 7.79 & 1.28 & .15 \\
\hline Pre-Reappraisal NA & 10.42 & 1.00 & 1.02 & .04 \\
\hline Pre-Suppression SBP & 118.45 & 15.31 & 10.86 & .68 \\
\hline Pre-Suppression DBP & 78.00 & 8.37 & 8.82 & .46 \\
\hline Pre-Suppression HR & 70.92 & 11.58 & & \\
\hline Pre-Suppression LF HRV & 2080.96 & 2162.09 & 3.17 & .35 \\
\hline Pre-Suppression HF HRV & 2066.24 & 2466.34 & 3.11 & .43 \\
\hline Pre-Suppression PA & 20.36 & 8.24 & 1.28 & .15 \\
\hline Pre-Suppression NA & 19.94 & 4.91 & 1.02 & .04 \\
\hline
\end{tabular}

*Note. SBP and DBP values are in mm Hg. HR is in bpm. LF HRV and HF HRV is in Hz. SBP and DBP are square-root transformed. HRV, PA, and NA are log transformed. HR is not transformed. 
Table 7

Means and Standard Deviations for Dependent Measures during Task (Fear Film) Periods

\begin{tabular}{|c|c|c|c|c|}
\hline & $\begin{array}{l}\text { Mean } \\
\text { (untransformed) }\end{array}$ & $\begin{array}{l}\text { SD } \\
\text { (untransformed) }\end{array}$ & $\begin{array}{l}\text { Mean } \\
\text { (transformed) }\end{array}$ & $\begin{array}{l}\text { SD } \\
\text { (transformed) }\end{array}$ \\
\hline Reappraisal SBP & 119.73 & 14.44 & 10.92 & .65 \\
\hline Reappraisal DBP & 80.83 & 8.80 & 8.98 & .48 \\
\hline Reappraisal HR & 72.14 & 12.12 & & \\
\hline Reappraisal LF HRV & 1874.42 & 1689.43 & 3.13 & .36 \\
\hline Reappraisal HF HRV & 1618.38 & 1199.10 & 3.07 & .38 \\
\hline Reappraisal PA & 19.94 & 4.91 & 1.29 & .10 \\
\hline Reappraisal NA & 16.78 & 6.43 & 1.20 & .15 \\
\hline Suppression SBP & 122.82 & 17.22 & 11.06 & .75 \\
\hline Suppression DBP & 81.41 & 8.83 & 9.01 & .48 \\
\hline Suppression HR & 72.00 & 16.15 & & \\
\hline Suppression LF HRV & 1629.55 & 1789.00 & 3.04 & .40 \\
\hline Suppression HF HRV & 21645.73 & 1971.31 & 2.91 & .74 \\
\hline Suppression PA & 20.36 & 4.72 & 1.30 & .10 \\
\hline Suppression NA & 18.24 & 7.36 & 1.23 & .17 \\
\hline
\end{tabular}

*Note. SBP and DBP values are in mm Hg. HR is in bpm. LF HRV and HF HRV is in Hz. SBP and DBP are square-root transformed. HRV, PA, and NA are log transformed. HR is not transformed. 
Table 8

Univariate Correlations between Covariates and Demographic Variables

\begin{tabular}{|c|c|c|c|c|}
\hline & Age & Sex & BMI & Total HLQ \\
\hline Pre-Re SBP & -.25 & $-.54 * *$ & $.40^{*}$ & .07 \\
\hline Pre-Re DBP & -.23 & $-.48 * *$ & $.37 *$ & .02 \\
\hline Pre-Re HR & -.16 & -.08 & $.41^{*}$ & .87 \\
\hline Pre-Re LF HRV & .02 & .23 & .01 & -.10 \\
\hline Pre-Re HF HRV & -.01 & .28 & -.06 & -.14 \\
\hline Pre-Re PA & $.37 *$ & .03 & $.37 *$ & .14 \\
\hline Pre-Re NA & .02 & -.33 & -.20 & -.003 \\
\hline Pre-Su SBP & -.27 & $-.57 * *$ & .12 & .26 \\
\hline Pre-Su DBP & -.22 & $-.39 *$ & .25 & .14 \\
\hline Pre-Su HR & -.07 & .09 & $.40 *$ & .16 \\
\hline Pre-Su LF HRV & .06 & -.11 & $-.36 *$ & .15 \\
\hline Pre-Su HF HRV & -.02 & .17 & -.22 & .009 \\
\hline Pre-Su PA & .29 & .25 & $.35 *$ & .10 \\
\hline Pre-Su NA & -.05 & -.15 & .01 & .08 \\
\hline
\end{tabular}

*Note. Univariate correlations between demographic variables and covariates. SBP and DBP are square-root transformed. HRV, PA, and NA are log transformed. Pre-reappraisal is abbreviated as Pre-Re; pre-suppression is abbreviated as Pre-Su. Total HLQ refers to the total score on the Healthy Lifestyle Questionnaire. *p $<.05 ;{ }^{* *} \mathrm{p} \leq .01$. 
Table 9

Univariate Correlations between Dependent and Demographic Variables

\begin{tabular}{|c|c|c|c|c|}
\hline & Age & Sex & BMI & Total HLQ \\
\hline Re SBP & -.23 & $-.45 *$ & .11 & .19 \\
\hline Re DBP & -.17 & -.27 & .24 & -.05 \\
\hline Re HR & -.02 & -.06 & $.50 * *$ & .02 \\
\hline Re LF HRV & .01 & -.12 & -.24 & .02 \\
\hline Re HF HRV & -.09 & .13 & -.19 & -.09 \\
\hline Re PA & .14 & -.09 & .14 & -.16 \\
\hline Re NA & -.11 & .15 & -.12 & .14 \\
\hline Su SBP & -.20 & $-.47 * *$ & .28 & .13 \\
\hline Su DBP & -.23 & $-.46^{*}$ & .28 & .04 \\
\hline Su HR & .05 & .05 & $.38 *$ & .03 \\
\hline Su LF HRV & .30 & .19 & $-.38 *$ & -.11 \\
\hline Su HF HRV & -.005 & .34 & -.12 & -.09 \\
\hline Su PA & .20 & -.19 & .14 & .19 \\
\hline Su NA & -.22 & -.14 & -.13 & .03 \\
\hline $\begin{array}{l}\text { *Note. Univa } \\
\text { square-root tr }\end{array}$ & HRV, & $\begin{array}{l}\text { ographic } \\
\text { are log }\end{array}$ & . Reapp & $\begin{array}{l}\text { P and DBP are } \\
\text { bbreviated as }\end{array}$ \\
\hline \multicolumn{5}{|c|}{ Re; suppression is abbreviated as Su. Total HLQ refers to the total score on the Healthy Lifestyle } \\
\hline & & & & \\
\hline
\end{tabular}


Table 10

Main Effects (Habitual Strategy and Instructional Set) and Interaction Effects for Habitual Reappraisers and Suppressors on Behavioral Observations

\begin{tabular}{llll}
\hline & $F$ & $d f$ & $P$ \\
\hline $\begin{array}{llll}\text { Look Away } \\
\text { Group }\end{array}$ & 1.36 & 1 & .25 \\
$\quad$ Instructions & .50 & 1 & .49 \\
Group X Instructions & 1.60 & 1 & .22 \\
Error & & 25 & \\
Sustained Look Away & & & \\
$\quad$ Group & 3.62 & 1 & .07 \\
Instructions & 3.43 & 1 & .08 \\
$\quad$ Group X Instructions & .42 & 1 & .52 \\
$\quad$ & & 25 & \\
$\quad$ Error & & &
\end{tabular}

Note. Look Away = number of times participants looked away for less than five seconds;

Sustained Look Away = the number of times participants looked away for more than five seconds. Data is untransformed. 
Table 11

Differences in Physiological and Affective Reactivity from Pre-Reappraisal Rest Periods to Reappraisal Task Periods

\begin{tabular}{|c|c|c|c|c|}
\hline & Mean & $S D$ & $t(\mathrm{df})$ & $p$ \\
\hline Pre-Reappraisal RP to Reappraisal TP SBP & & & $-2.13(26)$ & .04 \\
\hline Pre-Reappraisal SBP & 10.79 & .71 & & \\
\hline Reappraisal SBP & 10.89 & .66 & & \\
\hline Pre-Reappraisal RP to Reappraisal TP DBP & & & $-2.08(26)$ & .05 \\
\hline Pre-Reappraisal DBP & 8.82 & .54 & & \\
\hline Reappraisal DBP & 8.92 & .47 & & \\
\hline Pre-Reappraisal RP to Reappraisal TP HR & & & $1.09(30)$ & .29 \\
\hline Pre-Reappraisal HR & 73.40 & 13.66 & & \\
\hline Reappraisal HR & 72.14 & 12.12 & & \\
\hline Pre-Reappraisal RP to Reappraisal TP LF HRV & & & $1.28(29)$ & .21 \\
\hline Pre-Reappraisal LF HRV & 3.21 & .44 & & \\
\hline Reappraisal LF HRV & 3.12 & .36 & & \\
\hline Pre-Reappraisal RP to Reappraisal TP HF HRV & & & $-.05(29)$ & .96 \\
\hline Pre-Reappraisal HF HRV & 3.06 & .46 & & \\
\hline Reappraisal HF HRV & 3.07 & .38 & & \\
\hline Pre-Reappraisal RP to Reappraisal TP PA & & & $-.15(31)$ & .88 \\
\hline Pre-Reappraisal PA & 1.28 & .15 & & \\
\hline Reappraisal PA & 1.29 & .10 & & \\
\hline Pre-Reappraisal RP to Reappraisal TP NA & & & $-6.46(31)$ & $\leq .001$ \\
\hline Pre-Reappraisal NA & 1.02 & .04 & & \\
\hline Reappraisal NA & 1.20 & .15 & & \\
\hline
\end{tabular}

*Note. Paired-samples t-test comparing physiological and affective reactivity during pre-reappraisal rest period and reappraisal task period. Pre-reappraisal is abbreviated as Pre-Re; Reappraisal is abbreviated as Re. SBP and DBP are square-root transformed. HRV, PA, and NA are log transformed. 
Table 12

Differences in Physiological and Affective Reactivity from Pre-Suppression Rest Periods to Suppression Task Periods

\begin{tabular}{|c|c|c|c|c|}
\hline & Mean & $S D$ & $t(\mathrm{df})$ & $p$ \\
\hline $\begin{array}{l}\text { Pre-Suppression RP to Suppression TP SBP } \\
\text { Pre- Suppression SBP }\end{array}$ & 10.88 & .69 & $-2.43(27)$ & .02 \\
\hline Suppression SBP & 11.04 & .76 & & \\
\hline $\begin{array}{l}\text { Pre-Suppression to Suppression TP DBP } \\
\text { Pre- Suppression DBP }\end{array}$ & $\begin{array}{l}8.84 \\
9.01\end{array}$ & $\begin{array}{l}.45 \\
.49\end{array}$ & $-4.52(27)$ & $\leq .001$ \\
\hline \multicolumn{5}{|l|}{ Suppression DBP } \\
\hline $\begin{array}{l}\text { Pre- Suppression RP to Suppression TP HR } \\
\text { Pre-Suppression HR }\end{array}$ & 70.92 & 11.59 & $-.64(31)$ & .53 \\
\hline Suppression HR & 72.00 & 16.15 & & \\
\hline $\begin{array}{l}\text { Pre- Suppression RP to Suppression TP LF HRV } \\
\text { Pre- Suppression LF HRV }\end{array}$ & 3.17 & .35 & $1.74(30)$ & .09 \\
\hline Suppression LF HRV & 3.04 & .40 & & \\
\hline $\begin{array}{l}\text { Pre- Suppression RP to Suppression TP HF HRV } \\
\text { Pre- Suppression HF HRV }\end{array}$ & 3.11 & .43 & $1.72(30)$ & .10 \\
\hline Suppression HF HRV & 2.91 & .74 & & \\
\hline $\begin{array}{l}\text { Pre- Suppression RP to Suppression TP PA } \\
\text { Pre- Suppression PA }\end{array}$ & 1.28 & .15 & $-.68(32)$ & .50 \\
\hline Suppression PA & 1.30 & .10 & & \\
\hline $\begin{array}{l}\text { Pre- Suppression to Suppression TP NA } \\
\text { Pre- Suppression NA }\end{array}$ & 1.02 & .04 & $-7.42(32)$ & $\leq .001$ \\
\hline Suppression NA & 1.23 & .17 & & \\
\hline
\end{tabular}

*Note. Paired-samples t-test comparing physiological and affective reactivity during pre-suppression rest period and suppression task period. Pre-suppression is abbreviated as Pre-Su; Suppression is abbreviated as Su. SBP and DBP are square-root transformed. HRV, PA, and NA are log transformed. 
Table 13

Main Effects and interaction Effects for Ease of Following Instructions and Ease of Regulating Emotions

\begin{tabular}{|c|c|c|c|}
\hline & $F$ & $d f$ & $P$ \\
\hline \multicolumn{4}{|l|}{ Regulate Emotions } \\
\hline Group & 1.01 & 1 & .32 \\
\hline Regulating & .27 & 1 & .61 \\
\hline Group X Regulating & .00 & 1 & .99 \\
\hline Error & & 27 & \\
\hline \multicolumn{4}{|l|}{ Follow Instructions } \\
\hline Group & .01 & 1 & .91 \\
\hline Regulating & 1.96 & 1 & .17 \\
\hline Group X Regulating & .35 & 1 & .56 \\
\hline Error & & 27 & \\
\hline
\end{tabular}


Table 14

Main Effects (Habitual Strategy and Instructional Set) and Interaction Effects for Habitual

Reappraisers and Suppressors Exposed to Fear Films while Reappraising and Suppressing:

Heart rate and Blood Pressure

\begin{tabular}{|c|c|c|c|}
\hline & $F$ & $D f$ & $p$ \\
\hline \multicolumn{4}{|l|}{ SBP (square-root) } \\
\hline Group & .98 & 1 & .33 \\
\hline Instructions & 3.55 & 1 & .04 \\
\hline Group X Instructions & .32 & 1 & .73 \\
\hline Error & & 22 & \\
\hline \multicolumn{4}{|l|}{ DBP (square-root) } \\
\hline Group & .09 & 1 & .77 \\
\hline Instructions & 3.37 & 1 & .04 \\
\hline Group X Instructions & .32 & 1 & .73 \\
\hline Error & & 22 & \\
\hline \multicolumn{4}{|l|}{ HR } \\
\hline Group & .32 & 1 & .58 \\
\hline Instructions & 1.1 & 1 & .36 \\
\hline Group X Instructions & .24 & 1 & .79 \\
\hline Error & & 28 & \\
\hline
\end{tabular}

*Note. Mixed factors ANCOVAs examining the main effects of group and instructions and the interaction effect of group and instructions for SBP, DBP, and HR. Pre-reappraisal and presuppression rest periods are controlled for in SBP, DBP, and HR analysis. Sex is also controlled for in SBP and DBP analysis. BMI is controlled for in the HR analysis. 
Table 15

Main Effects (Habitual Strategy and Instructional Set) and Interaction Effects for Habitual

Reappraisers and Suppressors Exposed to Fear Film while Reappraising and Suppressing:

Heart Rate Variability Measures

$F \quad d f \quad p$

\begin{tabular}{lccc}
\hline LF HRV (log) & 1.21 & 1 & .28 \\
Group & 1.31 & 1 & .26 \\
Instructions & .32 & 1 & .57 \\
Group X Instructions & & 27 & \\
Error & .51 & 1 & .48 \\
HF HRV (log) & 2.61 & 1 & .12 \\
Group & 2.79 & 1 & .11 \\
Instructions & & 27 & \\
Group X Instructions & & \\
Error
\end{tabular}

*Note. Mixed factors ANCOVAs examining the main effects of group and instructions and the interaction effect of group and instructions for LF and HF HRV. 
Table 16

Main Effects (Habitual Strategy and Instructional Set) and Interaction Effects for Habitual

Reappraisers and Suppressors Exposed to Fear Film while Reappraising and Suppressing:

Measures of Affect

$F \quad d f \quad p$

\begin{tabular}{|c|c|c|c|}
\hline PA (log) & & & \\
\hline Group & .00 & 1 & .95 \\
\hline Instructions & .86 & 1 & .36 \\
\hline Group X Instructions & .63 & 1 & .43 \\
\hline Error & & 29 & \\
\hline NA (log) & & & \\
\hline Group & .04 & 1 & .83 \\
\hline Instructions & 2.70 & 1 & .11 \\
\hline Group X Instructions & 1.28 & 1 & .27 \\
\hline Error & & 29 & \\
\hline
\end{tabular}


Figure 1.

Mediation of the Stress - Disease Relation by the Acute Stress Response

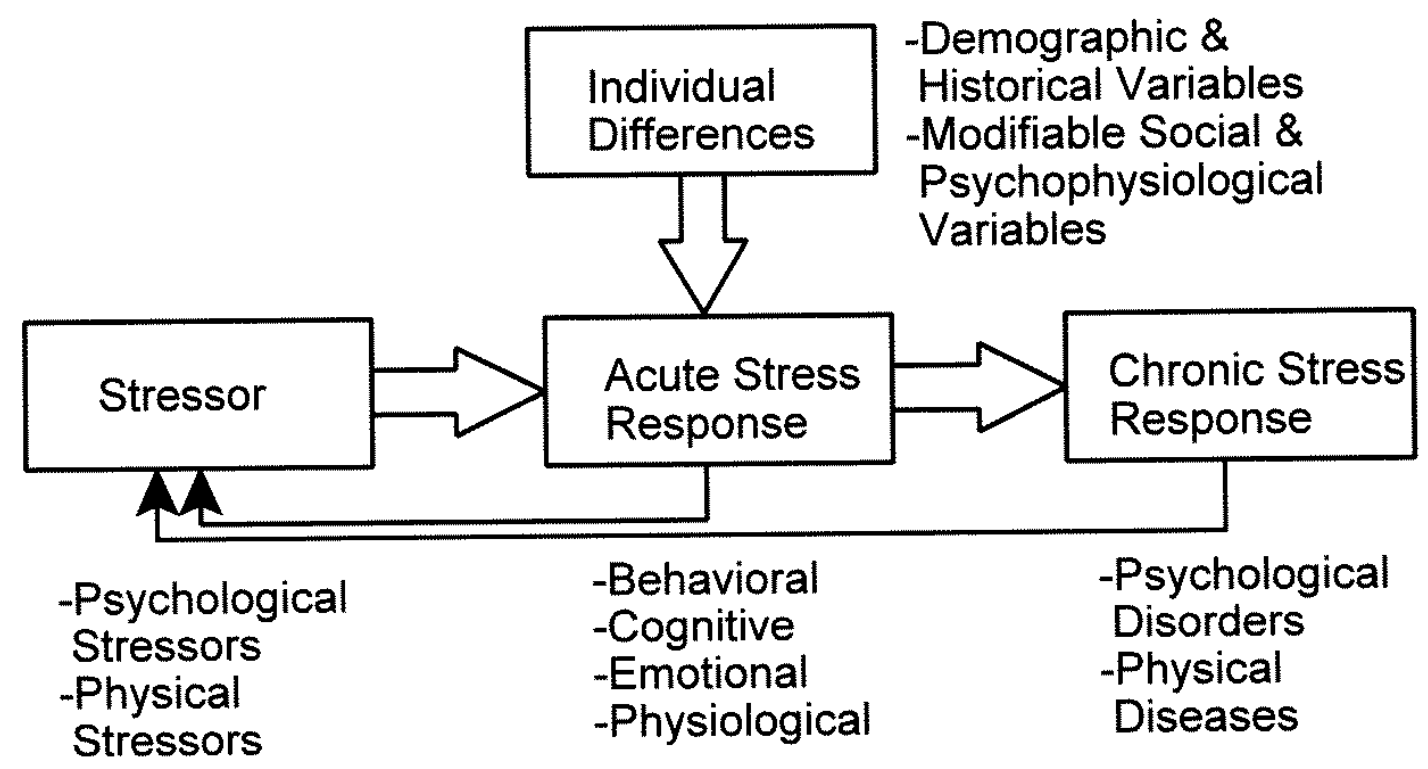

Larkin (2005). Stress and Hypertension. 
Figure 2.

Habitual Reappraisers (Upper Tertile and Lower Tertile Criterion)

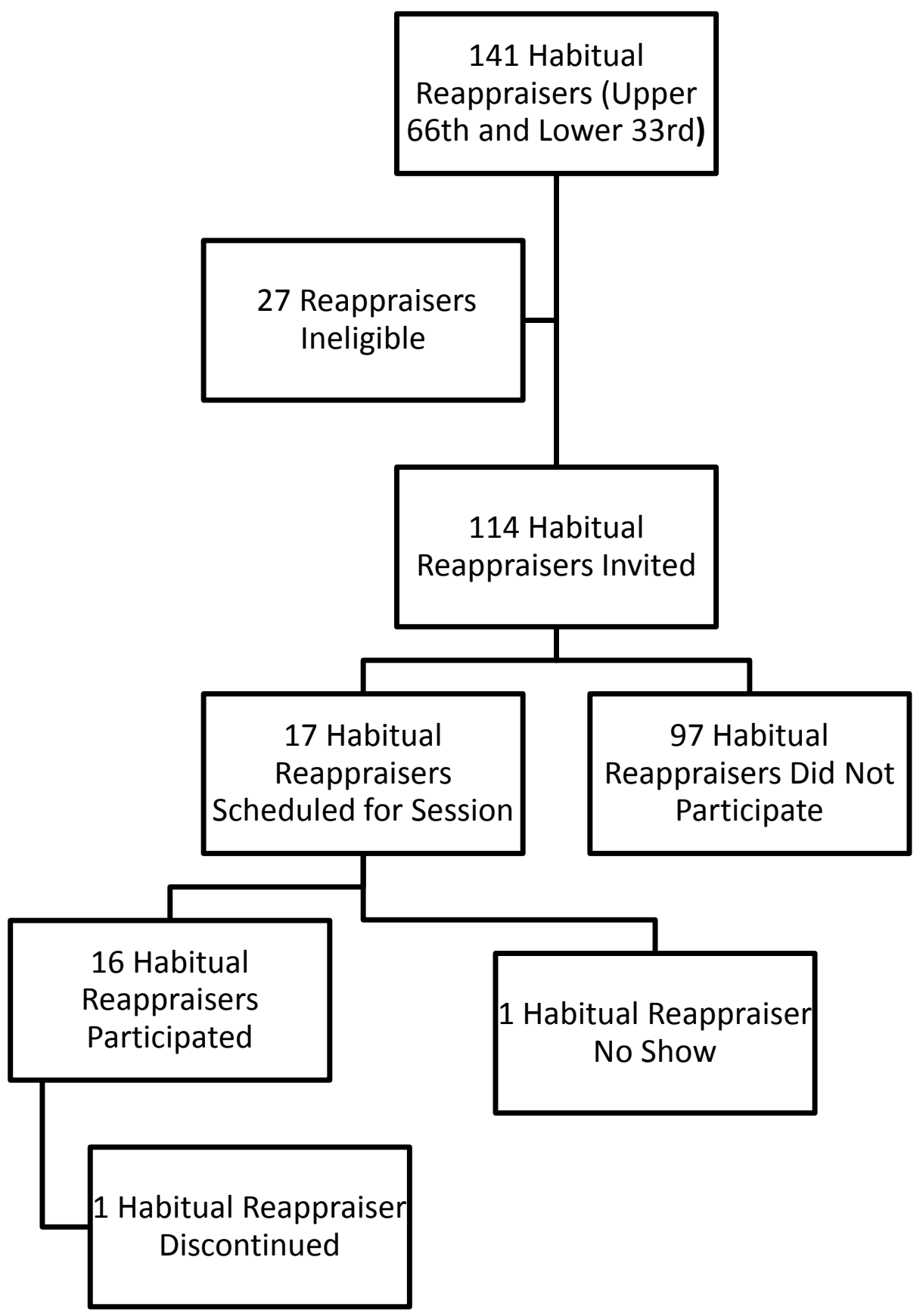


Figure 3.

Habitual Suppressors (Upper Tertile and Lower Tertile Criterion)

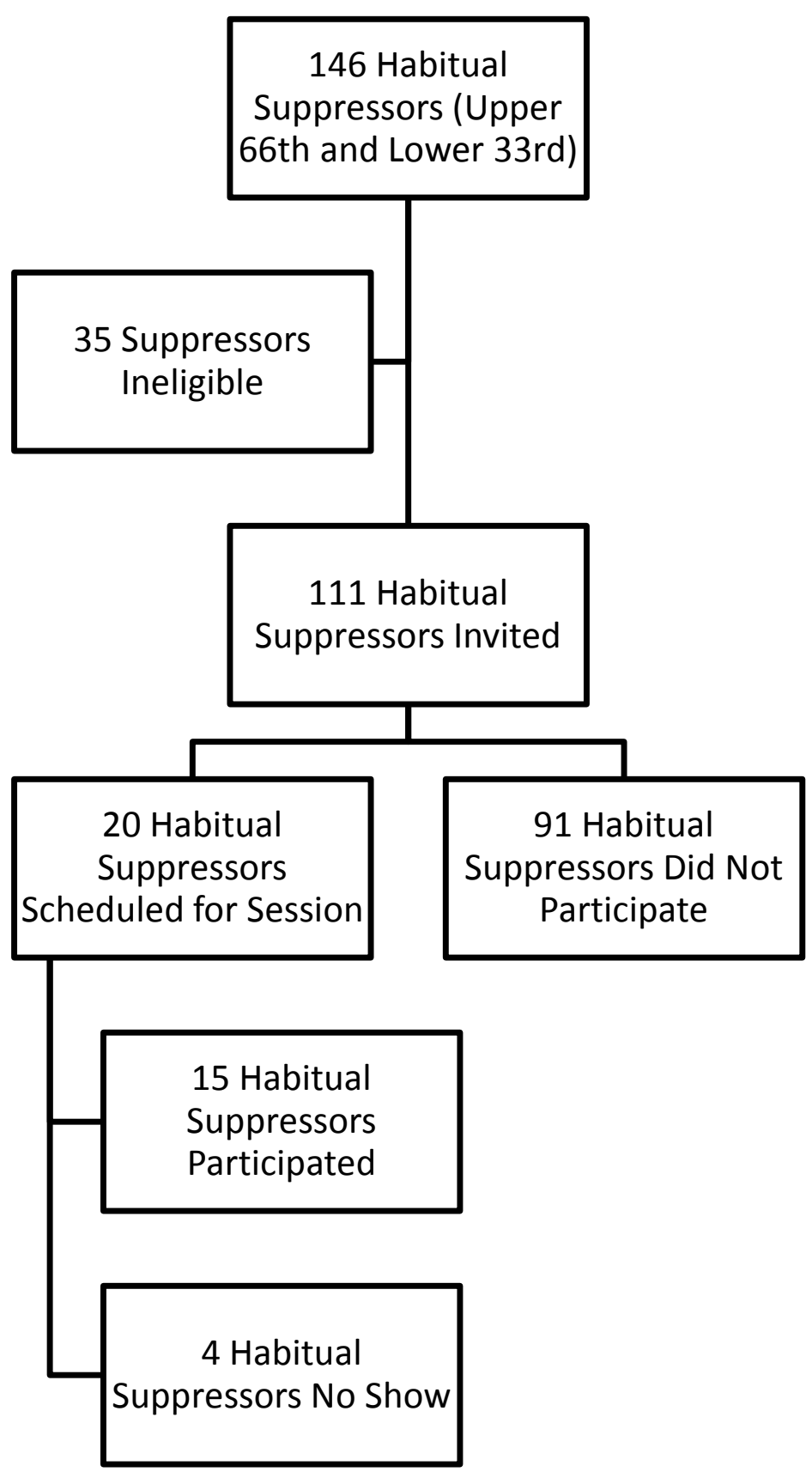


Figure 4.

Habitual Reappraisers (Upper Tertile and Lower $50^{\text {th }}$ Percentile Criterion)

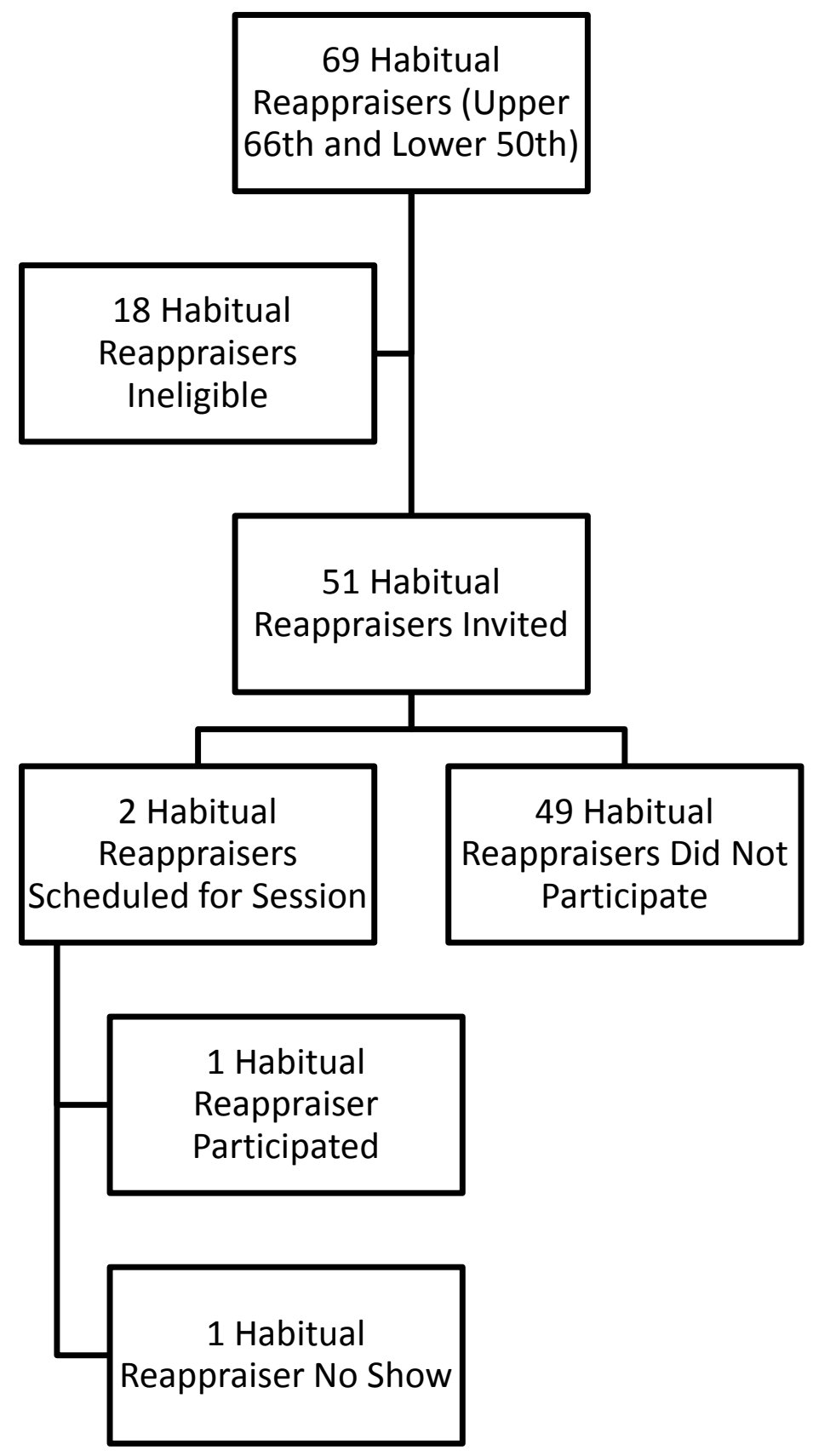


Figure 5.

Habitual Suppressors (Upper Tertile and Lower $50^{\text {th }}$ Percentile Criterion)

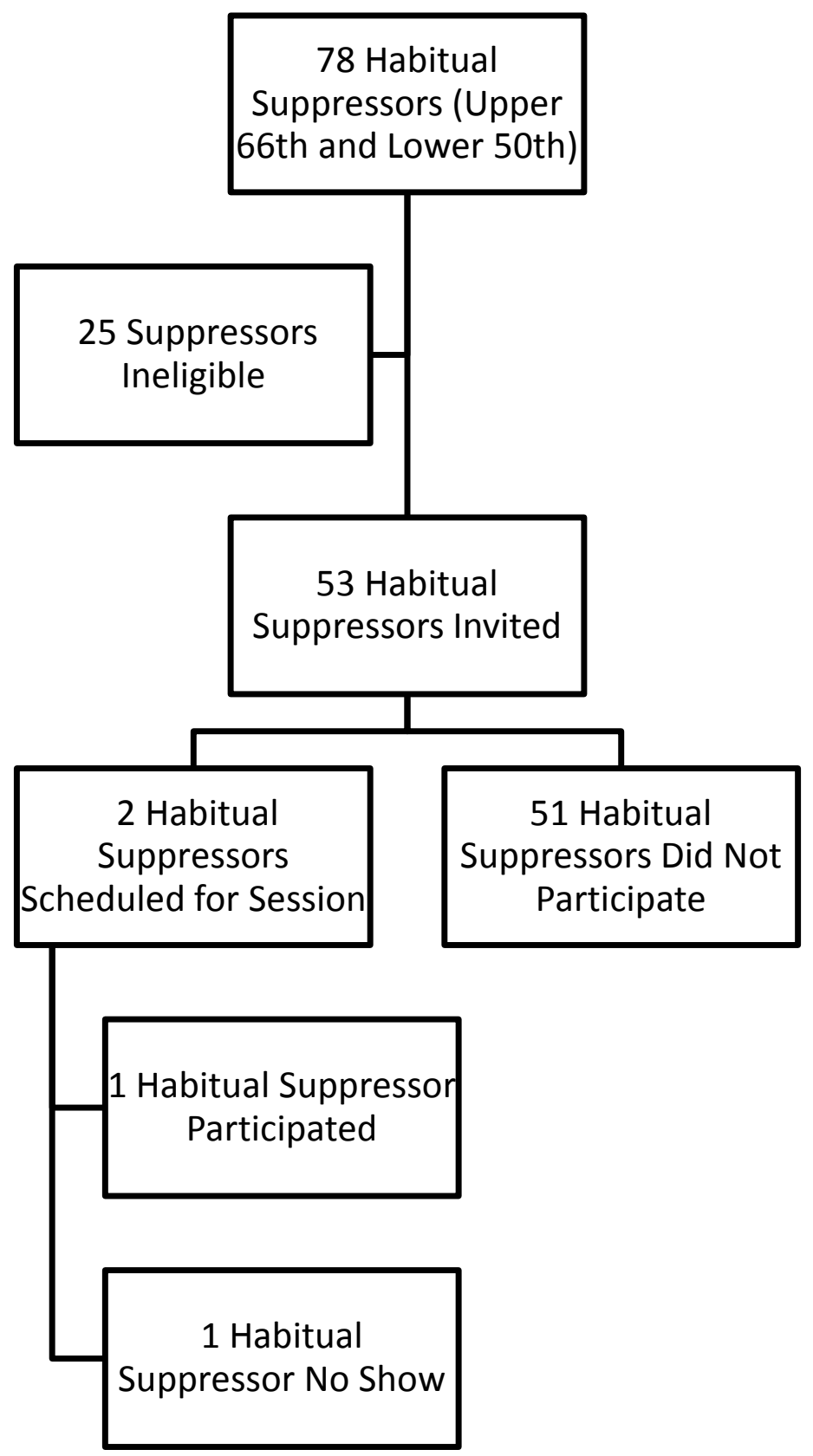


Figure 6.

Results of Block Randomization of Males and Females Categorized as Habitual Reappraisers and Habitual Suppressors into Conditions in Lab Phase

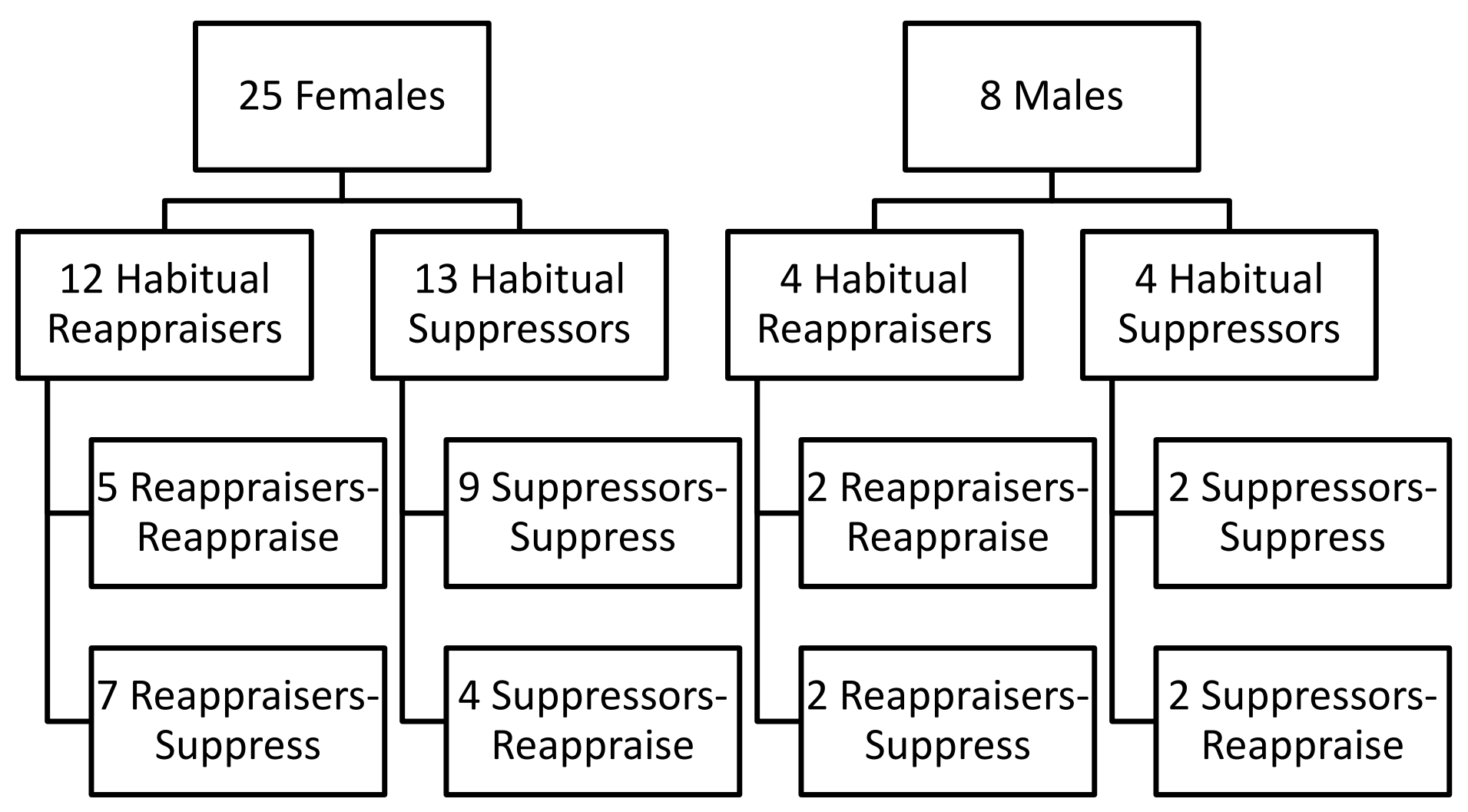

*Note . Reappraise $=$ Received Reappraisal instructions first; Suppress $=$ Received Suppression instructions first. 
Figure 7.

Procedure for Lab Phase

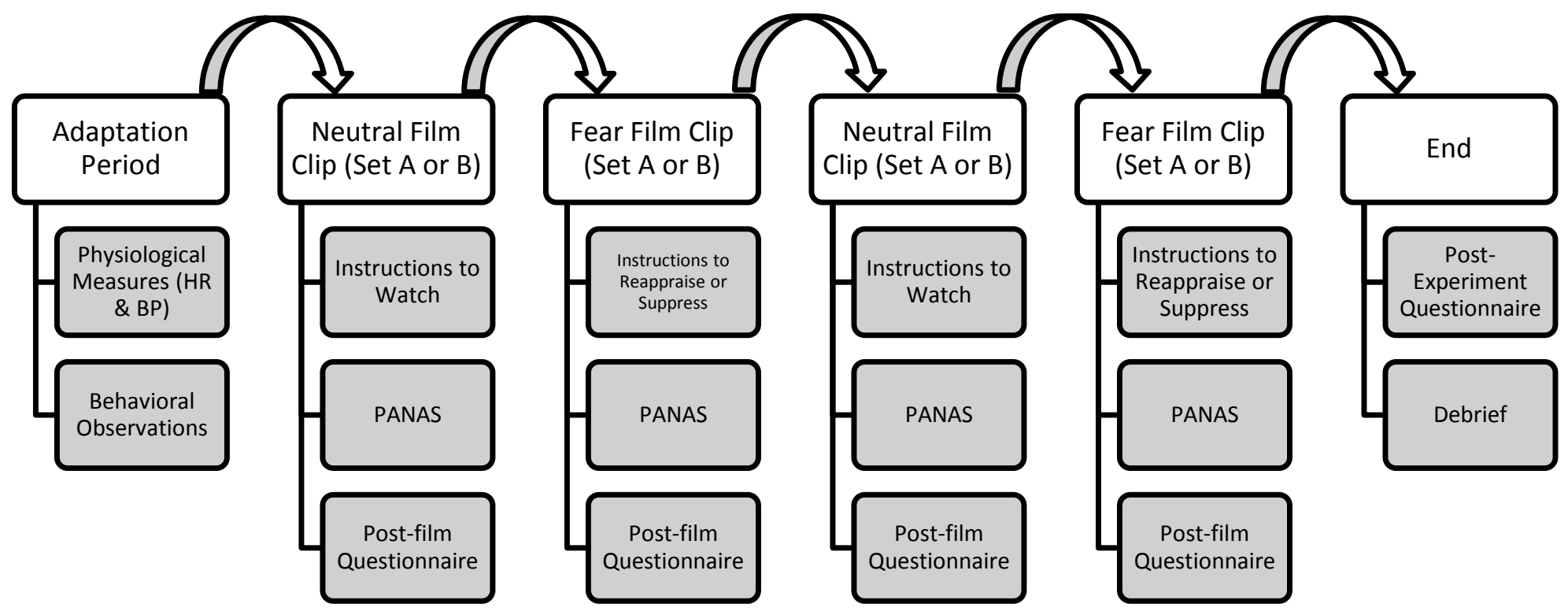




\section{Appendix A}

\section{Demographics Questionnaire}

\section{Participant \#:}

Height(in.):

\section{Your Information:}

Your age

Your sex

○ Male

$\circ$ Female

Your race

o Black

$\circ$ White

$\circ$ Asian

○ Biracial (specify):

o Other

Your ethnicity

○ Not Hispanic or Latino

○ Hispanic or Latino

Total years of education you have completed:

$\circ$ High school

○ 1 year college

$\circ 2$ years college

○ 3 years college

○ 4 or more years college
Date:

Weight(lbs): 
Please describe any cardiovascular related illness that you may have, including high blood pressure:

Please list any other medical or psychiatric problems that you have:

Please list any major surgeries and medical, or psychiatric illnesses you have had in the past.

Females: When did you start your last menstrual cycle?

○ I am a Male

○ less than one week ago

$\circ$ one week ago

○ two weeks ago

$\circ$ three weeks ago

$\circ$ four weeks ago

$\circ$ more than four weeks ago

$\circ$ I am currently pregnant

Females: Are you currently on birth control (contraceptives).

○ I am a male

$\circ$ No

$\circ$ Yes

What type of birth control are you taking?

Please list any drugs (legal or otherwise) that you are currently taking including; birth control (contraceptives), heart medications, cold or allergy medications, over the counter medications, asthma medications, Beta-Blockers (i.e. Inderal, Tenormin), psychoactive drugs (i.e. Adderall, Xanax, Haldol, Lithium, Prozac), or diet pills.

On average, how often do you smoke cigarettes? 
o never

○ I am not currently smoking

○ less than one pack per day

○ 1-2 packs per day

○ 2-3 packs per day

$\circ$ greater than 3 packs per day

On average, how often do you use smokeless tobacco?

o never

O I am not currently using smokeless tobacco

○ 1-4 times per day

○ 5-8 times per day

○ 9-13 times per day

$\circ$ greater than thirteen times per day

How often do you drink alcohol?

o never

$\circ$ infrequently (a few drinks per year)

○ occasionally (1-2 drinks per month)

○ weekly (1-3 drinks per week)

○ weekly (3-6 drinks per week)

○ daily (7-14 drinks per week)

$\circ$ daily (more than 14 drinks per week)

How many cups of caffeinated coffee, tea, soda, or energy drinks (with taurine) do you have per day?

o none

o 1-2 cups per day

○ 3-4 cups per day

- 5-6 cups per day

○ 7-8 cups per day 
$\circ$ greater than eight cups per day

About how many caffeinated beverages do you usually have by this time of day?

How many times per week do you engage in aerobic physical activity?

o never

○ 1-2 times

o 3-6 times

○ 7 or more times

For how long do you typically exercise on each occasion?

○ 5-10 minutes

○ 10-15 minutes

o 15-30 minutes

○ 30-60 minutes

o more than 60 minutes

How many hours of sleep did you get last night?

$\circ$ Less than 4 hours

○ 4-5 hours

○ 5-6 hours

○ 6-7 hours

○ 7-8 hours

o 8-9 hours

$\circ$ greater than 9 hours

\section{Family Information:}

What is your best estimate of your family's total income?

○ Less than 24,999

○ 25,000 to 34,999

○ 35,000 to 49,999

○ 50,000 to 74,999 
○ 75,000 to 99,999

○ 100,000 to 149,999

○ Greater than 150,000

Approximately how old is your father?

Is he currently living?

$\circ$ yes

$\circ$ no

Did/does your father have high blood pressure (hypertension)?

$\circ$ yes

o no

How certain are you that he did, or did not, have high blood pressure (hypertension)?

○ Absolutely (100\%) certain

$\circ$ Almost $(75 \%)$ certain

$\circ$ Not sure at all $(25 \%)$

○ No information by which to judge $(0 \%)$

Did/does your father have any heart problems such as angina (chest pains), a heart attack, or coronary heart disease?

○ yes

$\circ$ no

If yes, please specify if you are able:

How certain are you that he did, or did not, have a heart problem as indicated above?

$\circ$ Absolutely $(100 \%)$ certain

○ Almost (75\%) certain

$\circ$ Not sure at all $(25 \%)$

○ No information by which to judge (0\%)

Did/does your father have diabetes?

$\circ$ yes

$\circ$ no 
How certain are you that he did, or did not, have diabetes?

○ Absolutely (100\%) certain

$\circ$ Almost (75\%) certain

○ Not sure at all (25\%)

○ No information by which to judge $(0 \%)$

Did/does your father have a kidney disease (other than kidney stones)?

$\circ$ yes

$\circ$ no

How certain are you that he did, or did not, have a kidney disease (other than kidney stones)?

$\circ$ Absolutely (100\%) certain

$\circ$ Almost (75\%) certain

o Not sure at all (25\%)

○ No information by which to judge $(0 \%)$

Did/does your father have cancer?

○ yes

○ no

How certain are you that he did, or did not, have cancer?

○ Absolutely (100\%) certain

$\circ$ Almost (75\%) certain

$\circ$ Not sure at all $(25 \%)$

○ No information by which to judge (0\%)

If you know, please list what type(s) of cancer he had? 
Approximately how old is your mother?

Is she currently living?

$\circ$ yes

○ no

Did/does your mother have high blood pressure (hypertension)?

○ yes

$\circ$ no

How certain are you that she did, or did not, have high blood pressure (hypertension)?

○ Absolutely (100\%) certain

○ Almost (75\%) certain

$\circ$ Not sure at all $(25 \%)$

○ No information by which to judge $(0 \%)$

Did/does your mother have any heart problems such as angina (chest pains), a heart attack, or coronary heart disease?

$\circ$ yes

○ no

If yes, please specify if you are able:

How certain are you that she did, or did not, have a heart problem as indicated above?

$\circ$ Absolutely $(100 \%)$ certain

$\circ \operatorname{Almost}(75 \%)$ certain

$\circ$ Not sure at all $(25 \%)$

○ No information by which to judge $(0 \%)$ 
Did/does your mother have diabetes?

○ yes

$\circ$ no

How certain are you that she did, or did not, have diabetes?

$\circ$ Absolutely (100\%) certain

○ Almost (75\%) certain

$\circ$ Not sure at all $(25 \%)$

$\circ$ No information by which to judge $(0 \%)$

Did/does your mother have a kidney disease (other than kidney stones)?

$\circ$ yes

○ no

How certain are you that she did, or did not, have a kidney disease (other than kidney stones)?

$\circ$ Absolutely (100\%) certain

○ Almost (75\%) certain

$\circ$ Not sure at all $(25 \%)$

$\circ$ No information by which to judge $(0 \%)$

Did/does your mother have cancer?

$\circ$ yes

$\circ$ no

How certain are you that she did, or did not, have cancer?

$\circ$ Absolutely (100\%) certain 
$\circ$ Almost (75\%) certain

$\circ$ Not sure at all $(25 \%)$

○ No information by which to judge $(0 \%)$

If you know, please list what type(s) of cancer she had? 


\section{Appendix B}

\section{Emotion Regulation Questionnaire (ERQ)}

The Emotion Regulation Questionnaire is designed to assess individual differences in the habitual use of two emotion regulation strategies: cognitive reappraisal and expressive suppression.

\section{Citation}

Gross, J.J., \& John, O.P. (2003). Individual differences in two emotion regulation processes: Implications for affect, relationships, and well-being. Journal of Personality and Social Psychology, 85, 348-362.

\section{Instructions and Items}

We would like to ask you some questions about your emotional life, in particular, how you control (that is, regulate and manage) your emotions. The questions below involve two distinct aspects of your emotional life. One is your emotional experience, or what you feel like inside. The other is your emotional expression, or how you show your emotions in the way you talk, gesture, or behave. Although some of the following questions may seem similar to one another, they differ in important ways. For each item, please answer using the following scale:

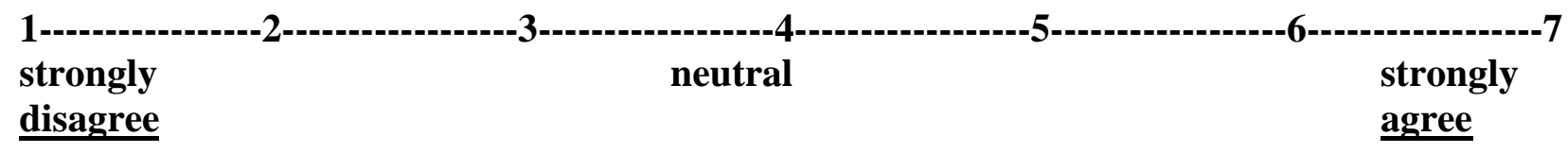

1. W__ When I want to feel more positive emotion (such as joy or amusement), I change what I'm thinking about.

2. ___ I keep my emotions to myself.

3. ___ When I want to feel less negative emotion (such as sadness or anger), I change what I'm thinking about.

4. ___ When I am feeling positive emotions, I am careful not to express them.

5. W_ When I'm faced with a stressful situation, I make myself think about it in a way that helps me stay calm.

6. ___ I control my emotions by not expressing them.

7. ___ When I want to feel more positive emotion, I change the way I'm thinking about the situation.

8. I I control my emotions by changing the way I think about the situation I'm in.

9. ___ When I am feeling negative emotions, I make sure not to express them. 
10. ___ When I want to feel less negative emotion, I change the way I'm thinking about the situation.

\section{Note}

Do not change item order, as items 1 and 3 at the beginning of the questionnaire define the terms "positive emotion" and "negative emotion".

\section{Scoring (no reversals)}

Reappraisal Items: 1, 3, 5, 7, 8, 10; Suppression Items: 2, 4, 6, 9. 


\section{Appendix C}

\section{Healthy Lifestyle Questionnaire}

For each item, please indicate how often you engage in each activity.

$$
\begin{aligned}
& 1=\text { Rarely/Never } \\
& 2=\text { Occasionally } \\
& 3=\text { Often } \\
& 4=\text { Always/Almost Always }
\end{aligned}
$$

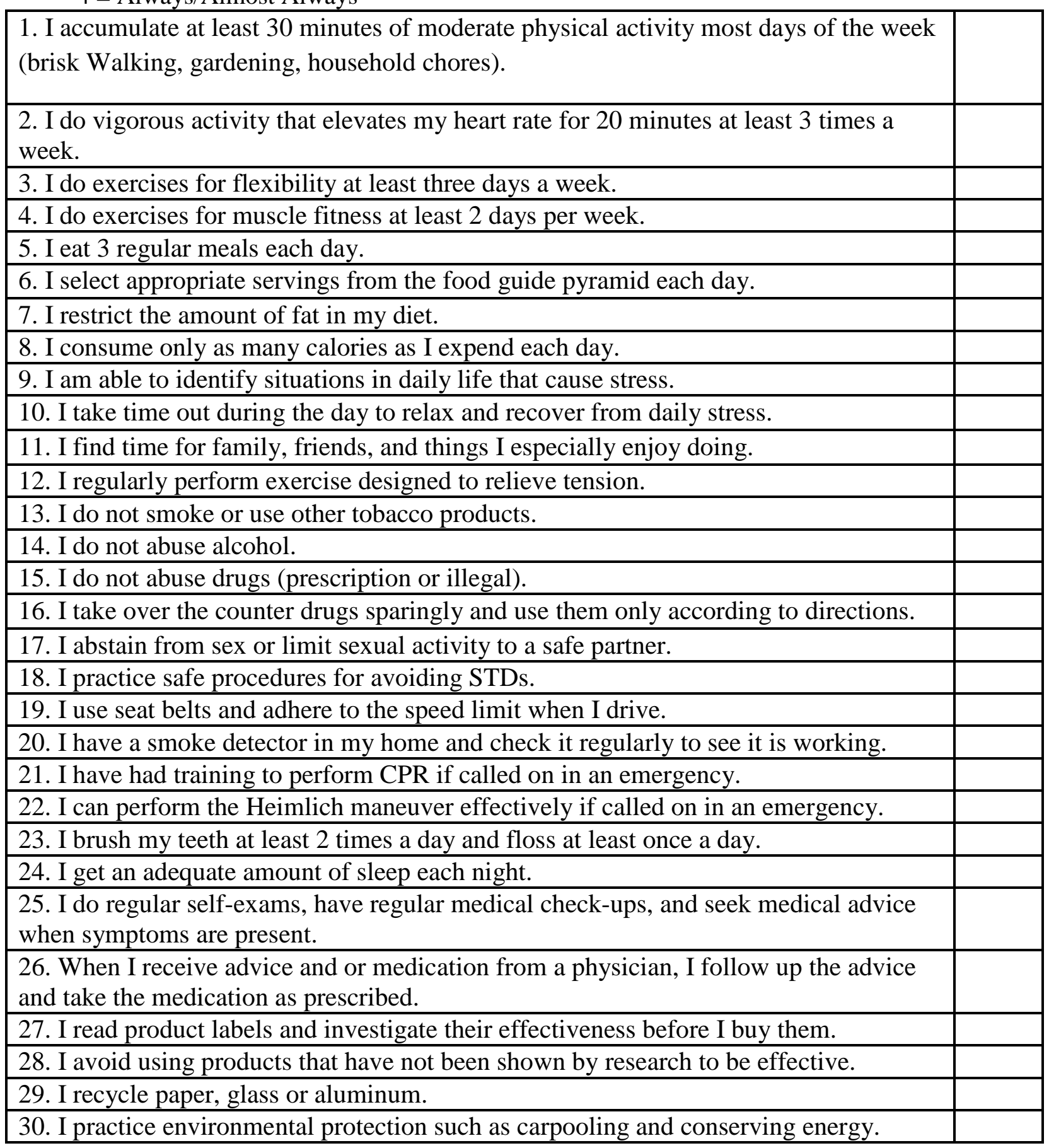


Scoring: Add point total for each answer.

\begin{tabular}{|l|l|}
\hline \multicolumn{1}{|c|}{ Subscale } & \multicolumn{1}{c|}{ Items } \\
\hline Physical Activity & $1,2,3,4$ \\
\hline Nutrition & $5,6,7,8$ \\
\hline Managing Stress & $9,10,11,12$ \\
\hline Avoiding Destructive Habits & $13,14,15,16$ \\
\hline Practice Safe Sex & 17,18 \\
\hline Adopting Safety Habits & 19,20 \\
\hline Knowing First Aid & 21,22 \\
\hline Personal Health Habits & 23,24 \\
\hline Using Medical Advice & 25,26 \\
\hline Being an Informed Consumer & 27,28 \\
\hline Protecting the Environment & 29,30 \\
\hline
\end{tabular}




\section{Appendix D}

Fear Survey Schedule II (FSS-II)

The items in this survey refer to things and experiences that may cause fear or other unpleasant feelings. In the space next to each item write the number which describes how much you are disturbed by it nowadays.

$\begin{array}{ll}1 & \text { None } \\ 2 & \text { Very little } \\ 3 & \text { A little } \\ 4 & \text { Some } \\ 5 & \text { Much } \\ 6 & \text { Very much } \\ 7 & \text { Terror }\end{array}$

1. Sharp objects

2. Being a passenger in a car

3. Dead bodies

4. Suffocating

5. Failing a test

6. Looking foolish

7. Being a passenger in an airplane

9. Arguing with parents

10. Rats and Mice

11. Life after death

12. Hypodermic needles

13. Being criticized

14. Meeting someone for the first time

15. Roller Coasters

16. Being alone

17. Making mistakes

18. Being misunderstood

19. Death

20. Being in a fight

21. Crowded places

22. Blood

23. Heights

24. Being a leader

25. Swimming alone

26. Illness

27. Being with drunks
28. Illness or injury to love ones

29. Being self-conscious

30. Driving a car

31. Meeting authority

32. Mental illness

33. Closed places

34. Boating

35. Spiders

36. Thunderstorms

37. Not being a success

38. God

39. Snakes

40. Cemeteries

41. Speaking before a group

42. Seeing a fight

43. Death of a loved one

44. Dark places

45. Strange dogs

46. Deep water

47. Being with a member of the opposite sex

48. Stinging insects

49. Untimely or early death

50. Losing a job

51. Auto accidents

52. Watching scary movies

53. Watching horror movies 


\section{Appendix E}

\section{PANAS}

This scale consists of a number of words that describe different feelings and emotions. Read each item and then mark the appropriate answer in the space next to that word. Indicate to what extent [INSERT APPROPRIATE TIME INSTRUCTIONS HERE]. Use the following scale to record your answers.

Copyright (C) 1988 by the American Psychological Association. Reproduced with permission. Watson, D., Clark, L. A., \& Tellegan, A. (1988). Development and validation of brief measures of positive and negative affect: The PANAS scales. Journal of Personality and Social Psychology, 54(6), 1063-1070.

1

very slightly

or not at all
2

a little
3

moderately
4

quite a bit
1. Interested

2. Distressed

3. Excited

4. Upset

5. Strong

6. Guilty

7. Scared

8. Hostile

9. Enthusiastic

10. Proud
11. Irritable

12. Alert

13. Ashamed

14. Inspired

15. Nervous

16. Determined

17. Attentive

18. Jittery

19. Active

20. Afraid

PANAS instructions:

Moment (you feel this way right now, that is, at the present moment)

Today (you have felt this way today)

Past few days (you have felt this way during the past few days)

Week (you have felt this way during the past week)

Past few weeks (you have felt this way during the past few weeks)

Year (you have felt this way during the past year)

General (you generally feel this way, that is, how you feel on the average) 


\section{Scoring Instructions:}

Positive Affect Score: Add the scores on items 1, 3, 5, 9, 10, 12, 14, 16, 17 , and 19 . Scores can range from $10-50$, with higher scores representing higher levels of positive affect. Mean Scores: Momentary _ 29.7 ( $S D_{-}$7.9); Weekly_33.3 ( $S D_{-}$. 7.2)

Negative Affect Score: Add the scores on items 2, 4, 6, 7, 8, 11, 13, 15, 18 , and 20. Scores can range from $10-50$, with lower scores representing lower levels of negative affect. Mean Score: Momentary _ 14.8 ( $S D_{-}$5.4); Weekly_ $17.4\left(S D_{-}\right.$6.2) 


\section{Appendix F}

\section{Post-film Questionnaire}

1. Have you previously seen this film clip?

2. Did you look away during the film clip?

$$
\text { Yes } \square \text { No }
$$

$$
\text { Yes } \square \text { No }
$$

3. Did you close your eyes during the film clip?

$$
\text { Yes } \square \text { No }
$$

4. What percentage of the time were your eyes open during the film?

$$
\begin{array}{llllllllll}
100 \% & 90 \% & 80 \% & 70 \% & 60 \% & 50 \% & 40 \% & 30 \% & 20 \% & 10 \%
\end{array}
$$

5. Please use the following scale to rate your pleasant feelings you experienced during the film clip. (Adapted from Kreibig et al., 2004)

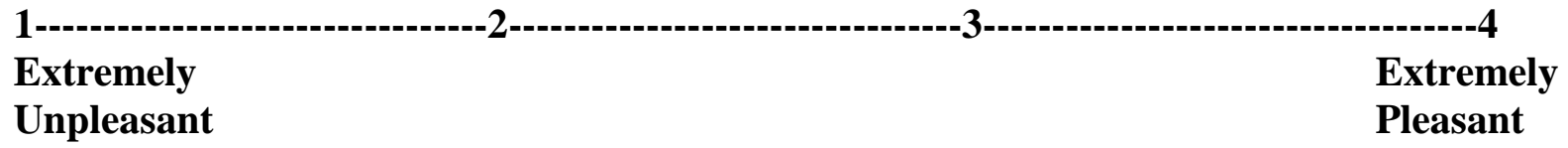

6. Please use the following scale to rate the intensity of feelings you experienced during the film clip. (Adapted from Kreibig et al., 2004)

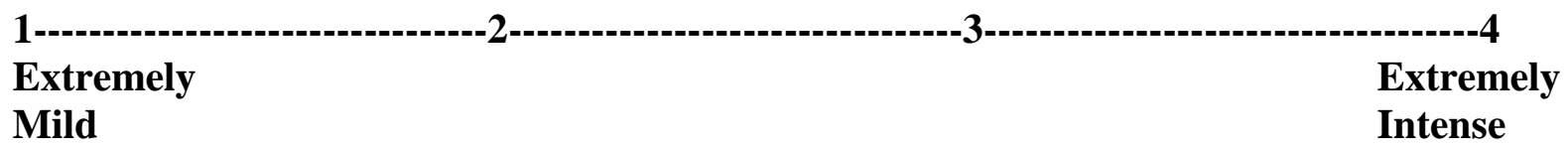

7. Please rate how easy it was to regulate your emotions during the film clip.

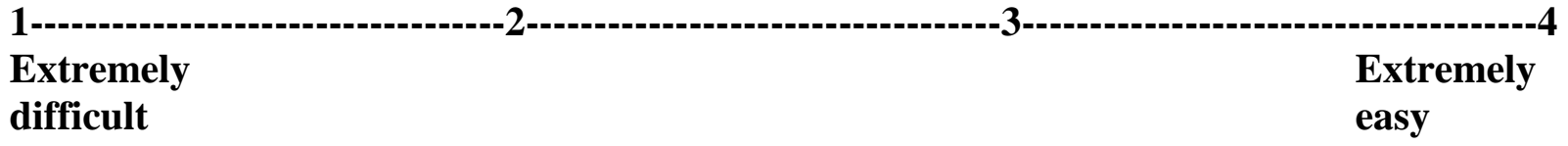

8. Please rate how easy it was to follow the instructions during the film clip.

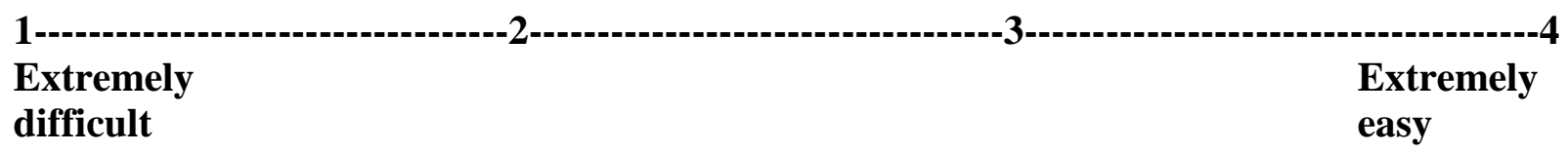




\section{Appendix G \\ Post-Experiment Questionnaire}

Please complete the questions below.

1. Please briefly describe your experience in this study.

2. Did you control your emotions better during I Know What You Did Last Summer film clip?

$\square$ Yes $\square$ No

3. Did you control your emotion betters during I Still Know What You Did Last Summer film clip?

$\square$ Yes

$\square$ No 


\section{Appendix $\mathrm{H}$}

Editing Instructions (Kreibig et al., 2007, p. 147-149 \& 152-153)

\section{Neutral Film I}

\subsection{Audio Introduction}

Number of words: 83 words

Audio length: $31 "$

Text: The next sequence is about Denali National Park, a preserve area in the Alaskan range. The park consists of wide, low plains, dark, somber mountains, brightly colored peaks and sheer granite dome. To see the park's beauty, a lot of visitors come there from all over the world. There is also a large variety of wildlife at Denali Park. In the first scene, you'll see a grizzly bear family, consisting of a mother bear and three young ones, at the beginning of spring.

\subsection{Film Clip}

Film: "Alaska's Wild Denali"

Target emotion: Neutral

\section{Clip length: 10'01"}

\section{Clip cutting:}

set time counter to 00:00:00:00 at first frame of film (mountain range in sunset light) right after "Alaska video postcards"

forward to 00:12:53:06; bear walking over snow field, speaker says, "Throughout the spring and summer the bears of Denali park are on a constant search for food"

end at 00:14:33:22; right before CUT to ground squirrel and speaker saying, "The arctic ground squirrel. . ."

forward to 00:21:50:28; camera panes from ducks to swans, “At least 159 bird species have been recorded in this area. . ."

end at 00:26:18:09; bear passes by yellow bus after speaker said, “. . . it affords the Denali visitors a unique chance to see the park's famous residents" and music

forward to 00:52:33:25; grizzly bear and three young ones on blueberry field; speaker says, "Against this fall back drop, the Denali grizzly bear family is on a picnic"

end at 00:56:25:26; after speaker said, “. . . and where we are able to watch, learn, and enjoy their behavior"; and right before the speaker says, "13 wolf packs use the Denali ecosystem. . . " and the wolf trots down the road 


\section{Neutral Film II}

2.1 Audio Introduction

Number of words: 83 words

Audio length: $30 "$

Text: Denali National Park is the setting of the following film cut. The Indians called the frozen peak of this great Alaskan mountain range Denali, or "the high one", commonly known as Mt McKinley. Visitors who see this impressing peak are very lucky, because it often is hidden by thick clouds. A lot of animals are the inhabitants of Denali National Park, among them moose, caribou, and Dall sheep. In the first scene, you'll see a flock of Dall sheep grazing on the mountainside.

\subsection{Film Clip}

Film: “Alaska's Wild Denali

Target emotion: Neutral

Clip length: 09'59"

\section{Clip cutting:}

set time counter to 00:00:00:00 at first frame of film (mountain range in sunset light) right after "Alaska video postcards"

forward to 00:15:53:21; camera zooming in on flower, then zooming in on sheep, "Dall sheep like to stick to the higher elevations to avoid predators below"

end at 00:19:23:05; cloud picture after eagle in the sky; right before CUT to mountain range

forward to 00:26:23:23; bus driving up hill; start right after background music faded; speaker says, "At Paligrown Pass, the park road narrows as it winds past layers of colorful volcanic rock. . ."

end at 00:30:48:00; tourist guide says, "Other co-workers have seen a wolf chasing a caribou here in the toe at"; just before CUT to ranger explaining sledge dog demonstration

forward to 00:46:11:18; moose and two calves grass next to a river, wade in it; speaker says, "The moose family does not appear to be bothered by the new conditions. . ."

end at 00:48:13:18; moose calves jumping and wading in water; right before CUT to grass, and then CUT to bird in tree, speaker saying, "The tigra forest, which dominates the landscape. .. "

END 


\section{Fear Film I}

3.1 Audio Introduction

Number of words: 84 words

Audio length: 28"

Text: In the following film cut, a teenage couple is stalked by a mysterious figure, which is clad in fisherman's garb and armed with a fishhook. Last year on July 4th, Helen earned the title of Croaker Queen in a beauty contest. A year later now, she is waiting to give the title to this year's beauty queen. Her boyfriend Barry who is trying to watch out for their tormentor, is attending the show. The first scene, you'll see is staged at the beauty contest.

\subsection{Film Clip}

Film: "I Know What You Did Last Summer"

Target emotion: Fear

Clip length: 10'39"

\section{Clip cutting:}

set time counter to 00:00:00:00 at the first frame where "A NEAL H. MORRITZ Production" can be read

forward to 01:10:46:12 for first scene (at the beauty contest); camera panes to Helen, contestant sings song "You ain't seen the best of me. . ."

end at 01:12:37:10; blood dripping from stair case pole; just before CUT to Julie arriving in her car at her home

forward to 01:12:40:15; right after CUT to Julie arriving in her car at her home ended; Helen sitting in foreground; policeman and woman discussing in background; policeman steps toward her and says, "Helen, I'm going to take you home. Your parents are really worried about you."

end at 01:13:08:08 Helen follows policeman; right before CUT to Julie at computer looking up newspaper article on David Egan in county library

forward to 01:13:14:16; police car driving through street lighted with street lamps; policeman says, "So then he killed him with a fish hook. . . "

end at 01:15:36:26 Fisherman chasing Helen; right before CUT to Julie reading up on Susie Willis trapped in car

forward to 01:15:49:03; Helen running away from fisherman, view of garage doors, window, and tree on right side

end at 01:20:34:25; Helen just ran around the corner after she jumped out of the window, seeing from the fisherman; right before CUT to Julie running into the empty hall where the beauty contest has been held 
forward to $01: 20: 41: 18$; Helen running up the stairs next to wooden boxes piled on top of each other, takes a glance over her shoulder to see whether she is followed

end at 01:21:53:12; Helen just got stabbed by the fisherman; camera panned away to view of tire piles; right before CUT to Julie in the crowd looking for her friends

END

\section{Fear Film II}

4.1 Audio Introduction

Number of words: 85 words

Audio length: 32"

Text: In the next sequence, Carla, playing a radio quiz with 96.7, wins a trip for four to the Bahamas, although she didn't give the right answer. Carla invites her boyfriend, Tie, and Julie and Will to come along. At the Bahamas, it's the last day of the season and a hurricane is coming. In the middle of this, random murders start to occur. In the next scene, you'll see Carla, Tie, and Julie in the hotel kitchen after accidentally breaking into another girl's hiding spot.

4.2 Film Clip

Film: "I Still Know What You Did Last Summer"

Target emotion: Fear

Clip length: 11'14"

\section{Clip cutting:}

set time counter to 00:00:00:00 at the first frame after CUT from statue of mother Mary to Julie walking down the aisle in church (starts right after title "I STILL KNOW WHAT YOU DID LAST SUMMER")

forward to 01:15:40:10; Julie, Karla, Nancy, and Tie are in the hotel kitchen; Nancy says, "So, that's what flashlights are for. . . "; right after CUT from Ray on water requesting emergency assistance

end at 01:22:49:15; Julie and Karla screaming after looking into two dead body's bloody faces (maid and other guy); right before CUT and close up to pool guy lying dead on floor

forward to 01:22:51:21; Julie, Karla, and Nancy still screaming after looking at dead man who wears red hotel uniform; right before Will opens door

end at 01:26:10:07; Will says, "I'm not doing well at school. . . ", end before Will says, "I'm having trouble with my boyfriend." 
forward to 01:26:11:22; right after Will said,’I'm having trouble with my boyfriend", just before Julie says "And I trusted you."

end 01:26:16:27; right after Will said, "You're like the rest of them. You never take responsibility for anything you do", right before he says, “. . . and you think you can get away with murder."

forward to 01:26:18:10; Julie pulls out knife, cutting Will; Will screams "What did you do this for?"

continue audio track until 01:27:06:20; right after Ben says, "No more screaming, no more running. It's time to die."

stop video track at 01:26:51:22; right when Ben pulls the cape from his head before his face/eyes can be seen

fill in video parts from 01:26:51:22 until 01:27:39:07 where Ben's face is not shown; views of Julie and Will

END 


\section{Appendix I}

Introduction to Film Clips (Kreibig, et al., 2004, p. 154-155)

\section{Clip 1 (Neutral) - Denali I}

“The next sequence is about Denali National Park, a preserve area in the Alaskan range. The park consists of wide, low plains, dark, somber mountains, brightly colored peaks, and sheer granite dome. To see the park's beauty, a lot of visitors come there from all over the world. There is also a large variety of wildlife at Denali Park. In the first scene you'll see a grizzly bear family, consisting of a mother bear and three young ones, at the beginning of spring." (83 words, 31 sec.)

\section{Clip 2 (Neutral) - Denali II}

"Denali National Park is the setting of the following film cut. The Indians called the frozen peak of this great Alaskan mountain range Denali, or "the high one," commonly known as Mount McKinley. Visitors who see this impressing peak are very lucky, because it often is hidden by thick clouds. A lot of animals are the inhabitants of Denali National Park, among them moose, caribou, and Dall sheep. In the first scene you'll see a flock of Dall sheep grazing on the mountainside.” (83 words, $30 \mathrm{sec}$.)

\section{Clip 3 (Fear) - I Know What You Did Last Summer}

"In the following film cut, a teenage couple is stalked by a mysterious figure, which is clad in fisherman's garb and armed with a fishhook. Last year on July 4th, Helen earned the title of Croaker Queen in a beauty contest. A year later now, she is waiting to give the title to this year's beauty queen. Her boyfriend Barry who is trying to watch out for their tormentor, is attending the show. The first scene you'll see is staged at the beauty contest.” (84 words, 28 sec.)

\section{Clip 4 (Fear) - I Still Know What You Did Last Summer}

"In the next sequence, Carla, playing a radio quiz with 96.7, wins a trip for four to the Bahamas, although she didn't give the right answer. Carla invites her boyfriend, Tie, and Julie and Will to come along. At the Bahamas it's the last day of the season and a hurricane is coming. In the middle of this, random murders start to occur. In the next scene, you'll see Carla, Tie, and Julie in the hotel kitchen after accidentally breaking into another girl’s hiding spot.” (85 words, 32 sec.) 